\title{
Introduction to bifurcation theory
}

\author{
John David Crawford \\ Institute for Fusion Studies, The University of Texas at Austin, Austin, Texas 78712 \\ and Department of Physics and Astronomy, University of Pittsburgh, Pittsburgh, Pennsylvania $15260^{\dagger}$
}

The theory of bifurcation from equilibria based on center-manifold reduction and Poincare-Birkhoff normal forms is reviewed at an introductory level. Both differential equations and maps are discussed, and recent results explaining the symmetry of the normal form are derived. The emphasis is on the simplest generic bifurcations in one-parameter systems. Two applications are developed in detail: a Hopf bifurcation occurring in a model of three-wave mode coupling and steady-state bifurcations occurring in the real Landau-Ginzburg equation. The former provides an example of the importance of degenerate bifurcations in problems with more than one parameter and the latter illustrates new effects introduced into a bifurcation problem by a continuous symmetry.

\section{CONTENTS}

I. Introduction

A. The basic setup

B. The basic question $\quad 994$

II. Linear Theory

A. Flows

1. Invariant linear subspaces

2. Hartman-Grobman theorem

Maps

1. Invariant linear subspaces

2. Hyperbolicity, Hartman-Grobman, and local bifurcation

III. Nonlinear Theory: Overview

IV. Persistence of Equilibria

A. Implicit function theorem

B. Applications to equilibria

1. Flows

2. Maps

V. Normal-Form Dynamics

A. Flows

1. Steady-state bifurcation: simple eigenvalue at zero

a. Saddle-node bifurcation: the typical case

b. Transcritical bifurcation: exchange of stability

c. Pitchfork bifurcation: reflection symmetry

2. Hopf bifurcation: a single conjugate pair of

B. Maps imaginary eigenvalues

1. Steady-state bifurcation: simple eigenvalue at $+1$

a. Saddle-node bifurcation

b. Transcritical bifurcation

c. Pitchfork bifurcation

2. Period-doubling bifurcation: a simple eigenvalue at -1

3. Hopf bifurcation: simple complex-conjugate pair at $|\lambda|=1$

C. Final remarks

VI. Invariant Manifolds for Equilibria

A. Flows

B. Maps

${ }^{*}$ Revised and expanded version of lectures delivered at the Institute for Fusion Studies at the University of Texas in 1989.

${ }^{\dagger}$ Permanent address.

1005

1006

1007

1007

1008

1009
VII. Center-Manifold Reduction

1009

A. Flows

1. Local representation of $W^{c}$

1009

2. The Shoshitaishvili theorem

3. Example

B. Maps; Local representation of $W^{c}$

C. Working on intervals in parameter space: suspended systems

VIII. Poincaré-Birkhoff Normal Forms

A. Flows

1. Generalities

2. Steady-state bifurcation on $\mathbb{R}$

3. Hopf bifurcation on $\mathbb{R}^{2}$

4. Normal-form symmetry

B. Maps

1. Generalities

2. Period-doubling bifurcation on $\mathbb{R}^{1}$

3. Hopf bifurcation on $\mathbb{R}^{2}$

IX. Applications

A. Hopf bifurcation in a three-wave interaction $\quad 1022$

1. Linear analysis

2. Approximating the center manifold

3. Determining the normal form

1022
1024

1024

B. Steady-state bifurcation in the Ginzburg-Landau equation

1. Bifurcation from $A=0$

a. $Q=0 \quad 1029$

b. $Q \neq 0$

2. A digression on phase dynamics 1030

3. Bifurcation from the pure modes 1031

a. Symmetry 1031

b. Linear stability for $a=0 \quad 1031$

c. Center-manifold reduction for $\lambda_{+} \approx 0 \quad 1032$

X. Omitted Topics 1033

Acknowledgments 1034

Index 1034

References 1035

\section{INTRODUCTION}

Bifurcation theory is a subject with classical mathematical origins, for example, in the work of $L$. Euler (1744); however, the modern development of the subject starts with Poincaré and the qualitative theory of differential equations. In recent years, this theory has undergone a tremendous development with an infusion of new ideas and methods from dynamical systems theory, singularity theory, group theory, and computer-assisted 
studies of dynamics. As a result, it is difficult to draw the boundaries of the theory with any confidence. The characterization offered twenty years ago by Arnold (1972) at least reflects how broad the subject has become:

The word bifurcation, meaning some sort of branching process, is widely used to describe any situation in which the qualitative, topological picture of the object we are studying alters with a change of the parameters on which the object depends. The objects in question can be extremely diverse: for example, real or complex curves or surfaces, functions or maps, manifolds or fibrations, vector fields, differential or integral equations.

In this review the "objects in question" will be dynamical systems in the form of differential equations and difference equations. In the sciences such dynamical systems commonly arise when one formulates equations of motion to model a physical system. The setting for these equations is the phase space or state space of the system. A point $x$ in phase space corresponds to a possible state for the system, and in the case of a differential equation the solution with initial condition $x$ defines a curve in phase space passing through $x$. The collective representation of these curves for all points in phase space comprises the phase portrait. This portrait provides a global qualitative picture of the dynamics, and this picture depends on any parameters that enter the equations of motion or boundary conditions.

If one varies these parameters the phase portrait may deform slightly without altering its qualitative (i.e., topological) features, or sometimes the dynamics may be modified significantly, producing a qualitative change in the phase portrait. Bifurcation theory studies these qualitative changes in the phase portrait, e.g., the appearance or disappearance of equilibria, periodic orbits, or more complicated features such as strange attractors. The methods and results of bifurcation theory are fundamental to an understanding of nonlinear dynamical systems, and the theory can potentially be applied to any area of nonlinear physics.

In Secs. II-VIII, we present a set of core results and methods in local bifurcation theory for systems that depend on a single parameter $\mu$. Here local bifurcation theory refers to bifurcations from equilibria where the phenomena of interest occur in the neighborhood of a single point. This restriction overlooks an extensive literature on global bifurcations where in some sense qualitative changes in the phase portrait occur that are not captured by looking near a single point. Wiggins (1988) provides an introduction to this aspect of the subject. $^{1}$ In addition, we shall concentrate on those bifurcations encountered in typical or "generic" systems. Thus

\footnotetext{
${ }^{1}$ It is worth emphasizing that the division between local and global bifurcations introduced here should not be taken too seriously. A detailed investigation of a global bifurcation often uncovers a rich spectrum of accompanying local bifurcations; similarly a local bifurcation of sufficient complexity can imply the occurrence of global bifurcations.
}

symmetric systems and Hamiltonian systems are not considered, with the exception of pitchfork bifurcation for reflection-symmetric systems. A precise mathematical description of generic can be given at the expense of introducing a number of technical definitions (Ruelle, 1989). The heuristic idea is simply that, when a parametrized system of equations exhibits a generic bifurcation, if we perturb the system slightly then the bifurcation will still occur in the perturbed system. One says that such a bifurcation is robust. Bifurcations that are robust in this sense for systems depending on a single parameter are referred to as codimension-one bifurcations. More generally, a codimension- $n$ bifurcation can occur robustly in systems with $n$ parameters but not in systems with only $n-1$ parameters. $^{2}$

The aim is to provide an accessible introduction for physicists who are not expert in dynamical systems theory, and an effort has been made to minimize the mathematical prerequisites. Consequently I begin with a summary of linear theory in Sec. II that includes the Hartman-Grobman theorem to underscore the link between linear instability and nonlinear bifurcation; this summary is supplemented in Sec. IV by an analysis of the persistence of equilibria using the implicit function theorem. The center-manifold-normal-form approach is outlined in Sec. III and developed in Secs. V-VIII.

Two applications of the theory are considered in Sec. IX. These illustrate the calculations required to reduce a specific bifurcation to normal form. In addition the examples offer a glimpse of several important and more advanced topics: new bifurcations that arise when there is more than one parameter, center-manifold reduction for infinite-dimensional systems, e.g., partial differential equations, and the effect of symmetry on a bifurcation.

Finally in Sec. $\mathrm{X}$ a brief survey of some topics omitted from this review is included for completeness and to provide some contact with current research areas in bifurcation theory. Our subject is very broad, and there is much activity by mathematicians, scientists, and engineers; the literature is enormous and widely scattered. This introduction does not attempt to assemble a comprehensive bibliography; the material of Secs. II-VIII can be found in many places, and in most cases the cited references are chosen simply because I have found them helpful. More extensive bibliographies can be found in the references.

\section{A. The basic setup}

It is advantageous to express different systems in a standard form so that the theory can be developed in a uniform way. As an example consider the second-order

\footnotetext{
${ }^{2}$ The geometric connotations of codimension can be made precise, but we do not require this development here (Arnold, 1988a). Roughly speaking, the set of systems associated with a codimension- $n$ bifurcation corresponds to a surface of codimension $n$.
} 
oscillator equation

$$
\ddot{y}+\dot{y}+y+y^{3}=0 ;
$$

by defining $x_{1} \equiv y$ and $x_{2} \equiv \dot{y}$, we can rewrite this evolution equation as a first-order system in two dimensions,

$$
\frac{d}{d t}\left(\begin{array}{l}
x_{1} \\
x_{2}
\end{array}\right)=\left[\begin{array}{c}
x_{2} \\
-x_{2}-x_{1}-x_{1}^{3}
\end{array}\right] \text {. }
$$

Clearly if higher-order derivatives in $t$ had appeared in Eq. (1.1a), we could still have obtained a first-order system by simply enlarging the dimension, e.g., defining $x_{3} \equiv \ddot{y}$; similarly, if the equations of motion had involved dependent variables in addition to $y(t)$, these could also have been incorporated by enlarging the dimension appropriately. As this example suggests, there is great generality in considering dynamical processes defined by first-order systems:

$$
\dot{x}=V(\mu, x), \quad x \in \mathbb{R}^{n}, \mu \in \mathbb{R},
$$

depending on a parameter $\mu$ and describing motion in an $n$-dimensional phase space $\mathbb{R}^{n}$. When formulated in this way a differential equation is identified with a vector field $V(\mu, x)$ on $\mathbb{R}^{n}$; conversely, given a vector field one can always define an associated differential equation. ${ }^{3}$

We shall also consider a second type of dynamics that represents the evolution of a system at discrete time intervals. In this case, the motion is described by a map,

$$
x_{j+1}=f\left(\mu, x_{j}\right), \quad x \in \mathbb{R}^{n}, \mu \in \mathbb{R},
$$

where $j=0,1,2, \ldots$ is the index labeling successive points on the trajectory. There are close connections between the dynamical systems defined by maps and vector fields. For example, in Eq. (1.2a), we may also think of solutions as trajectories: an initial condition $x(0)$ uniquely determines a solution $x(t)$, and the corresponding curve in $\mathbb{R}^{n}$ (parametrized by $t$ ) is the trajectory of $x(0)$. More abstractly, the association $x(0) \rightarrow x(t)$ defines a mapping

$$
\phi_{t}: \mathbb{R}^{n} \rightarrow \mathbb{R}^{n}
$$

where $\phi_{t}(x(0))=x(t)$. This mapping is called the flow determined by Eq. (1.2a).

In each case, the dynamics is allowed to depend on an adjustable parameter $\mu$, and the origin $(\mu, x)=(0,0)$ is assumed to be an equilibrium or fixed point for the motion,

$$
V(0,0)=0 \text {, }
$$

or

\footnotetext{
${ }^{3}$ One often wishes to consider phase spaces more general than $\mathbb{R}^{n}$, for example, finite-dimensional manifolds such as tori or spheres. However, in these cases the dynamics on a neighborhood of a fixed point can be described by the models we consider by introducing a local coordinate system.
}

$$
f(0,0)=0 .
$$

Note that given a fixed-point solution $\left(\mu_{0}, x_{0}\right)$ one can always move it to the origin by a change of coordinates, so the representation in Eq. (1.3) is quite general.

The theory we develop for maps $(1.2 \mathrm{~b})$ is useful in a variety of circumstances. Two particularly important applications are to bifurcations from periodic orbits of differential equations and in the related context of bifurcations in systems that are periodically forced. Let $x_{\tau}(t)$ denote a periodic solution to Eq. (1.2a) with period $\tau$, i.e., $x_{\tau}(t)=x_{\tau}(t+\tau)$; the dynamics near $x_{\tau}(t)$ can be analyzed by constructing the Poincare return map. Let $\Sigma$ denote an $n-1$ dimensional plane in $\mathbb{R}^{n}$ which intersects $x_{\tau}(t)$ at the point $p$ (see Fig. 1). To define the return map $f$, consider a point $\sigma \in \Sigma$ near $p$, and solve Eq. (1.2a) using $\sigma$ as an initial condition. For $\sigma$ sufficiently near $p$, the trajectory from $\sigma$ will intersect $\Sigma$ at some new point $\sigma^{\prime}$; this intersection defines the action of the map $f$ on $\sigma$,

$$
\sigma^{\prime}=f(\sigma) \text {. }
$$

This definition is sensible for all points on $\Sigma$ in an appropriate neighborhood of $p$. Notice that $p$ is a fixed point for $f, f(p)=p$, since $x_{\tau}$ is a periodic orbit.

In the second application, a periodic modulation is applied to the system in Eq. (1.2a) so that $V(\mu, x)$ is replaced by

$$
\dot{x}=V(\mu, x, t), \quad x \in \mathbb{R}^{n}, \mu \in \mathbb{R}
$$

and

$$
V(\mu, x, t)=V(\mu, x, t+\tau),
$$

where $\tau$ is the period of the modulation. In this circumstance it is convenient to introduce the "stroboscopic" map $f$ by, in effect, recording the state of the system only once during each period of the modulation. More precisely, fix a definite time $t_{0}$ and then choose any initial condition $x_{0} \in \mathbb{R}^{n}$. Let $x\left(t ; t_{0}\right)$ denote the solution with the initial condition $x\left(t_{0} ; t_{0}\right)=x_{0}$, and define $f$ by

$$
x_{j+1}=f\left(x_{j}\right), j=0,1,2, \ldots
$$

where $x_{j}=x\left(t_{0}+j \tau ; t_{0}\right)$. The qualitative properties of the map $f(x)$ in Eq. (1.6) are independent of the specific choice $t_{0}$ used in the definition. Furthermore, fixed

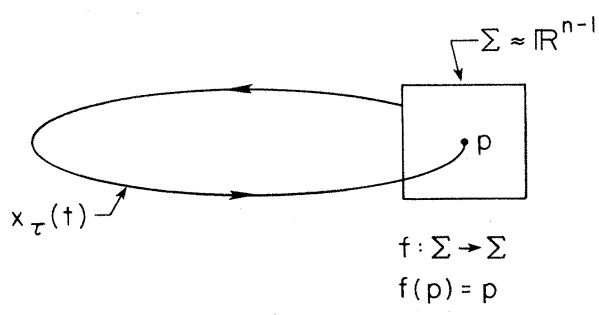

FIG. 1. Poincaré return map for a periodic orbit. 
points (1.3a) for the unmodulated system typically persist as fixed points for the map (1.6), at least for weak modulation. $^{4}$

\section{B. The basic question}

According to Eq. (1.2), at $\mu=0$ there is an equilibrium state at $x=0$. The basic question in local bifurcation theory is

What can happen in phase space near $x=0$ when there are variations in $\mu$ about $\mu=0$ ?

The Hartman-Grobman theorem, described in the next section, effectively reduces this question to an analysis of a narrower issue:

As $\mu$ is varied near $\mu=0$, what happens near $x=0$ if the stability of the equilibrium changes?

Before addressing this question, which involves the nonlinear terms of Eq. (1.2) in an essential way, it is necessary to develop the theory of linear stability.

\section{LINEAR THEORY}

\section{A. Flows}

At $x=0$ the Taylor expansion of Eq. (1.2a) begins,

$$
\dot{x}=V(\mu, 0)+D_{x} V(\mu, 0) \cdot x+\mathcal{O}\left(x^{2}\right),
$$

where $D_{x} V(\mu, 0)$ represents the square matrix with elements

$$
\left(D_{x} V(\mu, 0)\right)_{i j} \equiv \frac{\partial V_{i}}{\partial x_{j}}(\mu, 0),
$$

and $\mathcal{O}\left(x^{2}\right)$ indicates higher-order terms that are at least quadratic in the components of $x$. When the context is clear we shall omit the subscript $x$ and write $D V(\mu, 0)$ or simply $D V$. At $\mu=0$ the constant term in Eq. (2.1) vanishes, and near $x=0$ we study the linearized system,

$$
\dot{x}=D V(0,0) \cdot x,
$$

ignoring momentarily the effects of the nonlinear terms.

In the typical situation the eigenvalues of $D V(0,0)$ are nondegenerate ${ }^{5}$ and this matrix can be diagonalized by a linear change of coordinates $x \rightarrow x^{\prime}$. This allows Eq. (2.3) to be reexpressed as

\footnotetext{
${ }^{4}$ More precisely, this is true for hyperbolic fixed points, as
defined in Sec. II.A.2, and follows from the averaging theorem

${ }^{4}$ More precisely, this is true for hyperbolic fixed points, as
defined in Sec. II.A.2, and follows from the averaging theorem (Guckenheimer and Holmes, 1986). ${ }^{5} \mathrm{~A}$ degenerate eigenvalue is one for which there are two or
more linearly independent eigenvectors or generalized eigenvec${ }^{5} \mathrm{~A}$ degenerate eigenvalue is one for which there are two or
more linearly independent eigenvectors or generalized eigenvectors.
}

$$
\frac{d}{d t}\left(\begin{array}{c}
x^{\prime} \\
x_{2}^{\prime} \\
\vdots \\
x_{n}^{\prime}
\end{array}\right)=\left(\begin{array}{cccc}
\lambda_{1} & 0 & \cdots & 0 \\
0 & \lambda_{2} & & \\
\vdots & & \ddots & \\
0 & & & \lambda_{n}
\end{array}\right)\left(\begin{array}{c}
x^{\prime} \\
x_{2}^{\prime} \\
\vdots \\
x_{n}^{\prime}
\end{array}\right) ;
$$

if the spectrum of $D V(0,0)$ includes complex-conjugate pairs of eigenvalues, then the corresponding new coordinate components $x_{i}^{\prime}$ will also be complex (Hirsch and Smale, 1974). The general solution $x^{\prime}(t)$ is obviously

$$
x^{\prime}(t)=\left(\begin{array}{c}
x_{1}^{\prime}(0) e^{\lambda_{1} t} \\
x_{2}^{\prime}(0) e^{\lambda_{2} t} \\
\vdots \\
x_{n}^{\prime}(0) e^{\lambda n t}
\end{array}\right)
$$

If $\operatorname{Re} \lambda_{i}<0$, then as $t \rightarrow \infty$, the $x_{i}^{\prime}$ component decays to zero; conversely, $\operatorname{Re} \lambda_{i}>0$ implies exponentially rapid growth of $x_{i}^{\prime}$.

\section{Invariant linear subspaces}

For each eigenvalue $\lambda$ of $D V(0,0)$, there is an associated subspace of $\mathbb{R}^{n}$ - the eigenspace $E_{\lambda}$. For simplicity we assume $D V(0,0)$ is diagonalizable; then our definition of $E_{\lambda}$ depends only on whether $\lambda$ is real or complex. The
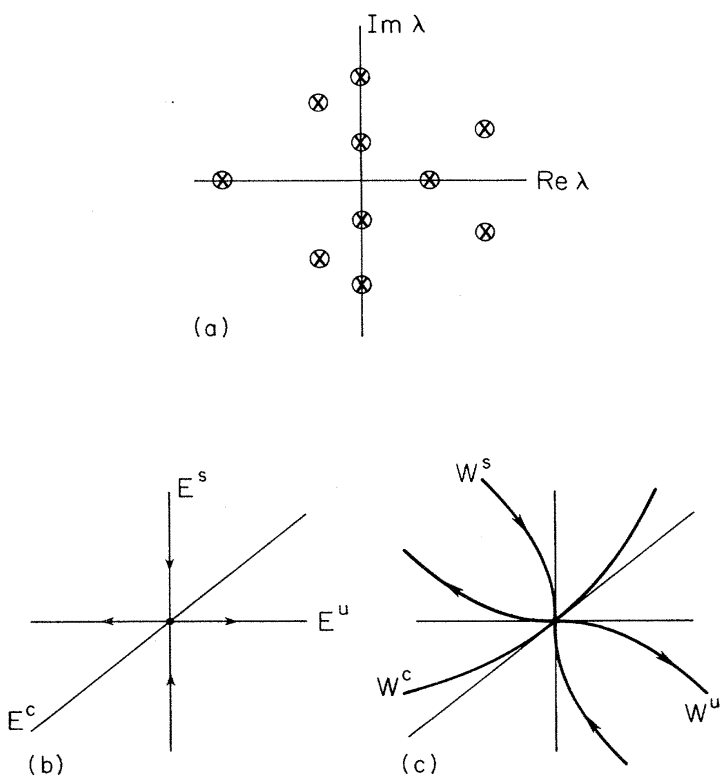

FIG. 2. Example of invariant subspaces and manifolds for a fixed point. (a) Linear spectrum showing stable modes, neutral modes, and unstable modes for an equilibrium $x=0$ in a flow; (b) invariant linear subspaces; for the spectrum in (a) we would have $\operatorname{dim} E^{s}=3, E^{c}=4, \operatorname{dim} E^{u}=3$; (c) invariant nonlinear manifolds; for the spectrum in (a) we would have $\operatorname{dim} W^{s}=3$, $\operatorname{dim} W^{c}=4, \operatorname{dim} W^{u}=3$. 
case of a real eigenvalue is most familiar. When $\lambda$ is real, $E_{\lambda}$ is simply the subspace spanned by the eigenvectors,

$\lambda \in \mathbb{R}, \quad E_{\lambda} \equiv\left\{v \in \mathbb{R}^{n} \mid(D V(0,0)-\lambda I) \cdot v=0\right\}$.

If $\lambda$ is nondegenerate, then we have $\operatorname{dim} E_{\lambda}=1$.

When $\lambda$ is complex, then the eigenvectors are also complex; furthermore, since $D V(0,0)$ is assumed to be a real matrix if $v_{1}+i v_{2}$ is the eigenvector for $\lambda$, the complex-conjugated vector $v_{1}-i v_{2}$ is an eigenvector for $\bar{\lambda}$. The eigenspace $E_{\lambda}$ in this case is spanned by the real and imaginary parts of the eigenvectors for $\lambda$, e.g., $v_{1}$ and $v_{2}$. Noting that both $v_{1}$ and $v_{2}$ satisfy $(D V(0,0)-\lambda I)(D V(0,0)-\bar{\lambda} I) \cdot v=0$, we replace Eq. (2.6a) with

$$
\lambda \notin \mathbb{R}, \quad E_{\lambda} \equiv\left\{v \in \mathbb{R}^{n} \mid(D V(0,0)-\lambda I)(D V(0,0)-\bar{\lambda} I) \cdot v=0\right\} .
$$

Now if $\lambda$ is nondegenerate we have $\operatorname{dim} E_{\lambda}=2$.

When $D V(0,0)$ has eigenvalues that are degenerate, this construction for $E_{\lambda}$ is satisfactory provided $D V(0,0)$ is diagonalizable. When $D V(0,0)$ cannot be diagonalized, then the definitions in Eq. (2.6) must be extended to include not only eigenvectors but generalized eigenvectors as well (Arnold, 1973; Hirsch and Smale, 1974).

An eigenvalue $\lambda$ corresponds to a "mode" of the system that is stable, unstable, or neutral, depending on whether $\operatorname{Re} \lambda<0, \operatorname{Re} \lambda>0$, or $\operatorname{Re} \lambda=0$, respectively [Fig. 2(a)]. We divide the eigenvectors (and generalized eigenvectors) of $D V(0,0)$ into three sets according to these possibilities and form the stable subspace $E^{s}$, unstable subspace $E^{u}$, and center subspace $E^{c}$.

$$
\begin{aligned}
& E^{s}=\operatorname{span}\left\{v \mid v \in E_{\lambda} \text { and } \operatorname{Re} \lambda<0\right\} \\
& E^{u}=\operatorname{span}\left\{v \mid v \in E_{\lambda} \text { and } \operatorname{Re} \lambda>0\right\}
\end{aligned}
$$
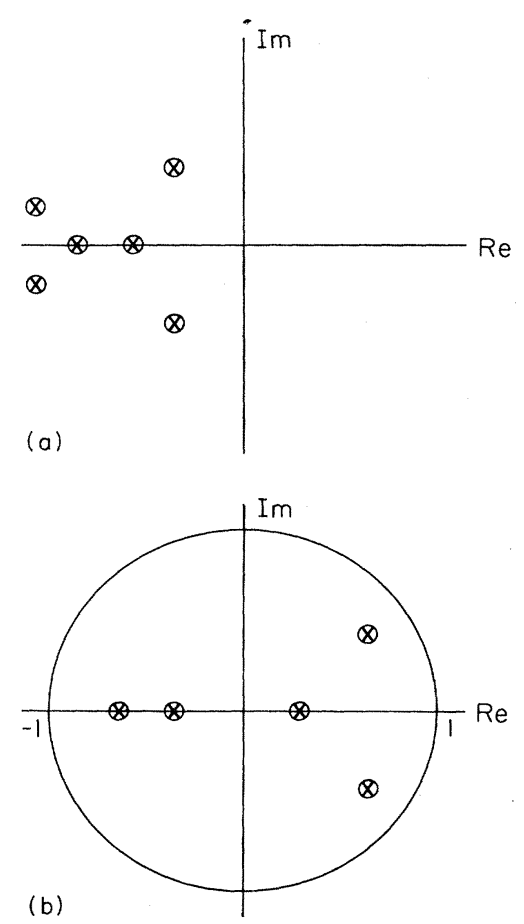

FIG. 3. A stable linear spectrum for a fixed point of (a) a flow and (b) a map.

$$
E^{c}=\operatorname{span}\left\{v \mid v \in E_{\lambda} \text { and } \operatorname{Re} \lambda=0\right\}
$$

These subspaces span the phase space, $\mathbb{R}^{n}=E^{s} \oplus E^{c} \oplus E^{u}$, and they are invariant: if $x(0) \in E^{\alpha}, \alpha=s, c, u$, then the trajectory $x(t)$ of Eq. (2.3) with this initial condition satisfies $x(t) \in E^{\alpha}$. For $E^{s}$ and $E^{u}$ the dynamics has a simple asymptotic description: if $x(t) \in E^{s}$, then as $t \rightarrow+\infty$ the trajectory converges to the equilibrium; if $x(t) \in E^{u}$, then the trajectory converges to the equilibrium as $t \rightarrow-\infty$. These features are illustrated in Fig. 2(b).

An equilibrium at $x=0$ is asymptotically stable if there exists a neighborhood of initial conditions, $0<|x(0)|<\varepsilon$, such that for all $x(0)$ in this neighborhood

(i) the trajectory $x(t)$ satisfies $|x(t)|<\varepsilon$ for $t>0$, and (ii) $|x(t)| \rightarrow 0$ as $t \rightarrow \infty$.
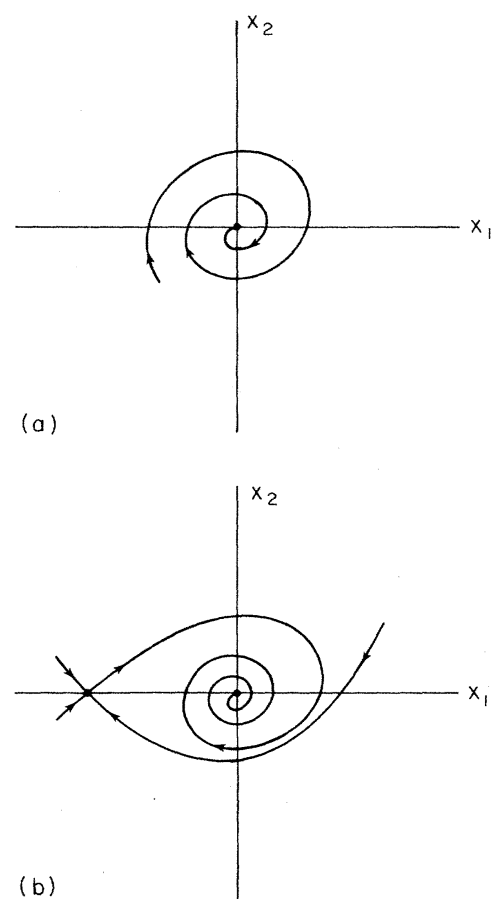

FIG. 4. Asymptotic stability of $x=0$. Such stability for the linear system (a) implies that $x=0$ is asymptotically stable for the nonlinear system $(b)$. 
For the linear system (2.3), the equilibrium $x=0$ is asymptotically stable if and only if $\operatorname{Re}(\lambda)<0$ for each eigenvalue $\lambda$ of $D V(0,0)$. In other words, the spectrum must lie within the left half-plane of the complex $\lambda$ plane [see Fig. 3(a)].

This criterion is particularly valuable because one can prove that if $x=0$ is asymptotically stable for Eq. (2.3), then it will also be asymptotically stable for the original nonlinear system (1.2a) (Hirsch and Smale, 1974). In Fig. 4(b) we show a schematic phase portrait for a twodimensional system with two fixed points on the $x_{1}$ axis. If we imagine linearizing about the stable equilibrium at the origin, then the resulting $2 \times 2$ matrix will have a complex-conjugate pair of eigenvalues $(\lambda, \bar{\lambda})$ satisfying $\operatorname{Re} \lambda=\operatorname{Re} \bar{\lambda}<0$. The phase portrait for the linearized system is shown in Fig. 4(a); the equilibrium $x=0$ is obviously asymptotically stable in Fig. 4(a) for arbitrarily large initial conditions. In the nonlinear phase portrait Fig. 4(b) $x=0$ is also asymptotically stable, but the neighborhood, $0<|x(0)|<\varepsilon$, of stable initial conditions is not arbitrarily large; it must not intersect the trajectories which are asymptotically drawn to the unstable fixed point on the negative $x_{1}$ axis. The linear test for asymptotical stability provides no information regarding the size of the neighborhood in the nonlinear system where the conclusion of stability holds.

\section{Hartman-Grobman theorem}

The qualitative relation between (2.3) and (1.2a) provided by the property of asymptotic stability is only applicable when all the eigenvectors are stable, i.e., when $E^{u}$ and $E^{c}$ are empty, but this instance does not exhaust the information about the nonlinear problem that is available from the linearized dynamics. Even if the equilibrium is not asymptotically stable, there are general theorems describing in what sense the qualitative features of Eq. (2.3) faithfully reflect the full nonlinear flow (1.2a) near $x=0$. For example, near a hyperbolic equilibrium, i.e., a fixed point with no eigenvalues on the imaginary axis, there exists a change of coordinates that transforms the nonlinear flow into the linear flow locally. Thus, even when there are unstable directions, the linearized dynamics remains a qualitatively accurate description of the nonlinear dynamics. The Hartman-Grobman theorem provides a precise statement of this idea. There is a generalization of this theorem due to Shoshitaishvili that treats the nonhyperbolic case when $E^{c}$ is not empty; this is discussed in Sec. VII.A.2.

Theorem II.1 (Hartman-Grobman). Let $x=0$ be a hyperbolic equilibrium for Eq. (1.2a) at some fixed value of $\mu$, $\phi_{t}$ denote the flow of $(1.2 a)$, and $\widetilde{\phi}_{t}$ denote the flow for the corresponding linear system:

$$
\dot{x}=D V(\mu, 0) \cdot x
$$

Then there exists a homeomorphism ${ }^{6}$

$$
\Psi: \mathbb{R}^{n} \rightarrow \mathbb{R}^{n}
$$

and a neighborhood $U$ of $x=0$ where ${ }^{7}$

$$
\phi_{t}(x)=\Psi^{-1} \circ \widetilde{\phi}_{t} \circ \Psi(x)
$$

for all $(x, t)$ such that $x \in U$ and $\phi_{t}(x) \in U$.

For a proof see Hartman (1982). Note that $\Psi(x)$ and its inverse cannot in general be assumed differentiable. In the terminology of dynamical systems, Eq. (2.8) defines a topological conjugacy (locally) between the linear flow and the nonlinear flow; this is a precise statement that the nonlinear dynamics near $x=0$ is qualitatively the same as the linear dynamics. In particular, if there are no unstable directions so that $\Psi(x)$ belongs to $E^{s}$, then $\widetilde{\phi}_{t} \circ \Psi(x) \rightarrow 0$ as $t \rightarrow \infty$ for the linear flow and Eq. (2.8) implies that $\phi_{t}(x) \rightarrow 0$ as $t \rightarrow \infty$ as well; i.e., linear asymptotic stability implies nonlinear asymptotic stability.

\section{Loss of hyperbolicity and local bifurcation}

The Hartman-Grobman theorem implies that any qualitative change or bifurcation in the local nonlinear dynamics must be reflected in the linear dynamics. If $x=0$ is hyperbolic, then the linearized dynamics is qualitatively characterized by the expanding and contracting flows on $E^{u}$ and $E^{s}$, respectively; this qualitative structure remains fixed unless the equilibrium loses its hyperbolicity. For this loss to occur, the eigenvalues of the stability matrix $D V$ must shift so as to touch the imaginary axis.

In Sec. IV we shall show that when a fixed point is hyperbolic, if $\mu$ is varied slightly near $\mu=0$, then the fixed point must persist, although its precise location in the phase space may shift. In this event the eigenvalues of the associated linear stability matrix $D V$ depend on $\mu$, and as the parameter value changes, it may happen that an eigenvalue $\lambda(\mu)$ approaches the imaginary axis. The system is said to be critical when $\operatorname{Re}(\lambda)=0$, and the corresponding parameter value $\mu=\mu_{c}$ belongs to the bifurcation set. This loss of hyperbolicity occurs in one of two ways, which we distinguish by the appearance of the spectrum at criticality ${ }^{8}$ :

\footnotetext{
${ }^{6} \mathrm{~A}$ homeomorphism is a continuous change of coordinates whose inverse is also continuous.

${ }^{7}$ Here the composition of functions $f(x)$ and $g(x)$ is written $f \circ g(x) \equiv f(g(x))$.

${ }^{8} \mathrm{~A}$ loss of hyperbolicity can readily involve more complicated scenarios if there are multiple parameters or if the problem has some special structure, e.g., if the equations are Hamiltonian or have symmetry.
} 
(1) A simple real eigenvalue at $\lambda=0$. We shall refer to this type of critical spectrum as steady-state bifurcation [see Fig. 5(a)]. The nonlinear behavior produced by steady-state bifurcation may take several forms, which we discuss in Sec. V. Most typical is saddle-node bifurcation, but in applications one also encounters transcritical bifurcation and pitchfork bifurcation.

(2) A simple conjugate pair of eigenvalues satisfying $\operatorname{Re} \lambda=\operatorname{Re} \bar{\lambda}=0$; see Fig. 5(b). This type of instability is commonly referred to as Hopf bifurcation [although the name does not reflect earlier work of Poincaré and Andronov (Arnold, 1988a)].

\section{B. Maps}

The corresponding linear theory for a map may be discussed in a similar fashion. The expansion of Eq. (1.2b) at $x=0$.

$$
x_{j+1}=f(\mu, 0)+D_{x} f(\mu, 0) \cdot x_{j}+\mathcal{O}\left(x^{2}\right),
$$

leads to the linearized system

$$
x_{j+1}=D f(0,0) \cdot x_{j}
$$

at $\mu=0$. As before, we diagonalized $D f(0,0)$ by changing coordinates $x \rightarrow x^{\prime}=\left(x_{1}^{\prime}, x_{2}^{\prime}, \ldots, x_{n}^{\prime}\right)$ and obtain
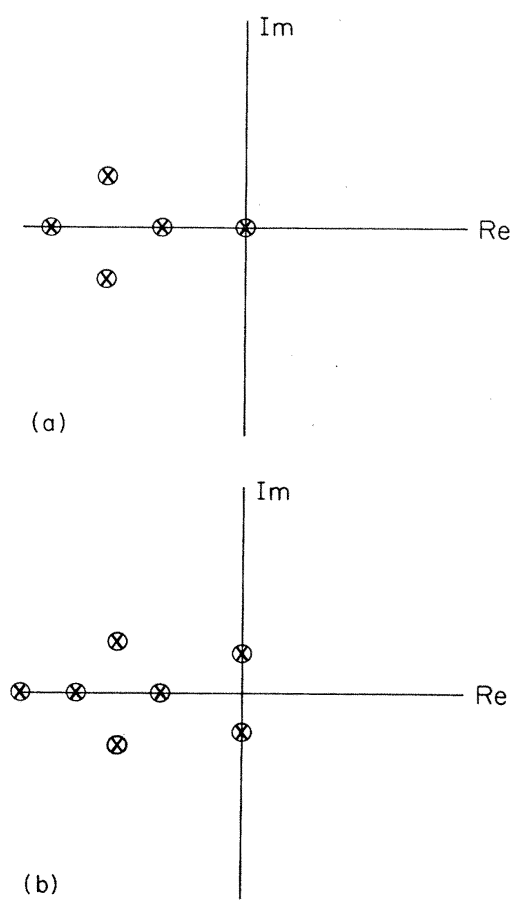

FIG. 5. Basic instabilities for an equilibrium in a flow: (a) steady-state bifurcation and (b) Hopf bifurcation.

$$
\left[\begin{array}{c}
x_{1}^{\prime} \\
x_{2}^{\prime} \\
\vdots \\
x_{n}^{\prime}
\end{array}\right]_{j+1}=\left(\begin{array}{cccc}
\lambda_{1} & 0 & \cdots & 0 \\
0 & \lambda_{2} & & \\
\vdots & & \ddots & \\
0 & & & \lambda_{n}
\end{array}\right]\left[\begin{array}{c}
x_{1}^{\prime} \\
x_{2}^{\prime} \\
\vdots \\
x_{n}^{\prime}
\end{array}\right]_{j},
$$

with solution

$$
\left[\begin{array}{c}
x_{1}^{\prime} \\
x_{2}^{\prime} \\
\vdots \\
x_{n}^{\prime}
\end{array}\right]_{j}=\left(\begin{array}{cccc}
\lambda_{1}^{j} & 0 & \cdots & 0 \\
0 & \lambda_{2}^{j} & & \\
\vdots & & \ddots & \\
0 & & & \lambda_{n}^{j}
\end{array}\right]\left[\begin{array}{c}
x_{1}^{\prime} \\
x_{2}^{\prime} \\
\vdots \\
x_{n}^{\prime}
\end{array}\right]_{0} .
$$

If $\left|\lambda_{i}\right|<1$, then as $j \rightarrow \infty$, the $x_{i}^{\prime}$ component decays exponentially; if $\left|\lambda_{i}\right|>1$, then the $x_{i}^{\prime}$ component will grow.

1. Invariant linear subspaces

For the linearized map (2.10) the eigenspace $E_{\lambda}$ for $D f(0,0)$ are defined as in Eq. (2.6) for the previous case by replacing $D V(0,0)$ with $D f(0,0)$. The invariant linear subspaces $E^{\alpha}, \alpha=s, u, c$, are defined as in Eq. (2.7), replacing $\operatorname{Re} \lambda$ by $|\lambda|-1$ to reflect the appropriate stability criteria,

$$
\begin{aligned}
& E^{s}=\operatorname{span}\left\{v \mid v \in E_{\lambda} \text { and }|\lambda|<1\right\}, \\
& E^{u}=\operatorname{span}\left\{v \mid v \in E_{\lambda} \text { and }|\lambda|>1\right\}, \\
& E^{c}=\operatorname{span}\left\{v \mid v \in E_{\lambda} \text { and }|\lambda|=1\right\} .
\end{aligned}
$$

As before, we have $\mathbb{R}^{n}=E^{s} \oplus E^{c} \oplus E^{u}$, and the stable and unstable subspaces have simple asymptotic dynamics as $j \rightarrow+\infty$ and $j \rightarrow-\infty$, respectively.

The definition of asymptotic stability given earlier applies to fixed points of maps provided $x(t)$ is replaced by $x_{j}$. For the linear dynamics (2.10), the equilibrium $x=0$ will be asymptotically stable if and only if the spectrum of $D f(0,0)$ lies within the unit circle in the complex $\lambda$ plane, i.e., $\left|\lambda_{i}\right|<1$ for each eigenvalue [see Fig. 3(b)]. It can be shown that if $x=0$ is asymptotically stable for Eq. (2.10) then the same conclusion holds for the full nonlinear dynamics (1.2b). In addition, for return maps (cf. Fig. 1), whose fixed points correspond to periodic orbits, the stability of a fixed point reflects the stability of the corresponding periodic orbit. [When the differential equation is linearized about the periodic orbit, the resulting linear equation may be analyzed using Floquet theory; the stability of the periodic orbit is determined from the spectrum of Floquet multipliers (Jordan and Smith, 1987). The eigenvalues of the return map linearized at the fixed point correspond to the Floquet multipliers of the periodic orbit.]

\section{Hyperbolicity, Hartman-Grobman,} and local bifurcation

As for flows, a fixed point is said to be hyperbolic if the center subspace $(2.13 c)$ is empty, and there is a 
Hartman-Grobman theorem relating the linearized dynamics to the local nonlinear dynamics: if, at $\mu=0, x=0$ is a hyperbolic fixed point, then there exists a homeomorphism $\Psi$ and a local neighborhood $U$ of $x=0$ where

$$
f(0, x)=\Psi^{-1}(D f(0,0) \cdot \Psi(x))
$$

for $x$ such that $x \in U$ and $f(0, x) \in U$.

If $x=0$ is a hyperbolic fixed point for $f(\mu, x)$ at $\mu=0$, then as $\mu$ is varied about zero this equilibrium will shift its location, but it will persist (see Sec. IV). The eigenvalues of $D f$ will be functions of $\mu$, and a variation in $\mu$ will cause them to move in the complex plane. If an eigenvalue reaches the unit circle, then the fixed point is no longer hyperbolic and a bifurcation can occur.

The possibilities may be classified by the form of the linear spectrum when the condition $\left|\lambda_{i}\right| \neq 1$ fails:

(1) A simple real eigenvalue at $\lambda=1$; see Fig. 6(a). This type of instability is quite similar to the $\lambda=0$ case for flows and is referred to as a steady-state bifurcation for maps. As in the case of flows, we find the saddle-node, transcritical, and pitchfork bifurcations as examples of steady-state bifurcation.

(2) A simple conjugate pair of eigenvalues $(\lambda, \bar{\lambda})$ where $\lambda=e^{i 2 \pi \theta}$; see Fig. 6(b). We shall refer to this case as Hopf bifurcation for maps to emphasize similarities with Hopf bifurcation in flows.
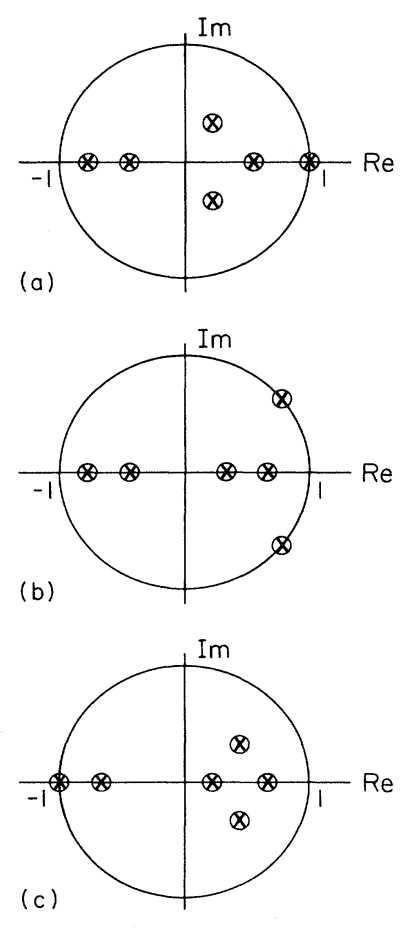

FIG. 6. Basic instabilities for an equilibrium in a map: (a) steady-state bifurcation; (b) Hopf bifurcation; (c) perioddoubling bifurcation.
(3) A simple real eigenvalue at $\lambda=-1$; see Fig. 6(c). This case is novel, as it does not have an analog in the earlier discussion of flows. This instability is generally termed period-doubling bifurcation, although the names flip bifurcation and subharmonic bifurcation are also used.

This completes our summary of linear stability theory and the forms of instability one typically expects to encounter when a single parameter is varied. Characterizing an instability by the form of the linear spectrum at criticality is more than a convenience; it is very advantageous to organize the theory (and one's understanding) in this way. The most important reason for this is that the linear spectrum determines the normal form. Precisely what this means will be explained in Sec. VIII.

\section{NONLINEAR THEORY: OVERVIEW}

Suppose an asymptotically stable equilibrium is perturbed by varying an external parameter $\mu$, and at a critical value $\mu=\mu_{c}$ the equilibrium develops a neutral mode ( $\operatorname{Re} \lambda=0$ for flows; $|\lambda|=1$ for maps). At $\mu_{c}$ hyperbolicity is lost, and we must study what happens to the system as $\mu$ is varied about $\mu_{c}$.

For all of the basic instabilities described in Sec. II, this issue can be investigated using the techniques of center-manifold reduction and normal-form theory. In brief outline, this approach has several steps:

(1) Reduction: identify the neutral mode (or modes) at $\mu=\mu_{c}$ and restrict the dynamical system to the appropriate center manifold;

(2) Normalization: if possible, put this reduced dynamical system into a simpler form by applying nearidentity coordinate changes. This yields the normal form for the bifurcation;

(3) Unfolding: describe the effects of varying $\mu$ away from $\mu_{c}$ by introducing small linear, and possibly nonlinear, terms into the normal form;

(4) Study the bifurcations described by the unfolded normal form. In this analysis, one truncates the unfolded system at some order and considers the resulting system. Once the truncated system is understood, the effect of restoring the higher-order terms can be discussed. ${ }^{9}$

The virtue of step one is that it reduces the dimension of the problem without any loss of essential information concerning the bifurcation. The advantages of the simplification offered in the second step are often decisive in being able to solve the problem. Furthermore, the re-

\footnotetext{
${ }^{9}$ In sufficiently complicated bifurcations, these effects can be significant and highly nontrivial. However, for most of the bifurcations considered in this review, these higher-order terms do not produce any qualitative changes. The one exception is Hopf bifurcation in maps, discussed in Sec. V.B.3.
} 
sulting simplified representation of the dynamics provides a universal, low-dimensional model for the given bifurcation.

This approach allows the general qualitative features of a bifurcation to be distinguished from specific quantitative aspects that will inevitably vary between different realizations of the bifurcation. The dimension of the reduced system and the structure of the appropriate normal form may be determined without requiring explicit evaluation of the coefficients in the normal form. Thus the variety of phenomena associated with a bifurcation can be described in a theory that is model independent. When this general theory is applied to a particular instability the normal-form coefficients can be calculated from the specific physical model under consideration. The possibility of determining the normal form without needing to derive the coefficients is often a considerable advantage.

In Sec. V, we present the normal forms for the bifurcations enumerated in Sec. II. Then the basic theory underlying the center-manifold reduction is discussed in Secs. VI and VII. Finally, in Sec. VIII, we develop the theory of Poincaré-Birkhoff normal forms and indicate how to derive the normal forms previously introduced in Sec. V.

In the next section, we consider a preliminary issue that it is useful to discuss before taking up the program outlined above. The question is basic: can the given equilibrium solution simply disappear when $\mu$ is varied? For both flows and maps, there are simple conditions on the linear spectrum that are sufficient to guarantee the persistence of an equilibrium.

\section{PERSISTENCE OF EQUILIBRIA}

\section{A. Implicit function theorem}

The implicit function theorem provides necessary conditions for an equilibrium of a flow or a map to disappear as $\mu$ varies. Equivalently these conditions can be restated as sufficient conditions for the equilibrium to persist. The following version of the theorem is adequate for our discussion; a proof may be found in Spivak (1965).

Theorem IV.1. Let $G(\mu, x)$ be a $C^{1}$ function on $\mathbb{R} \times \mathbb{R}^{n}$,

$$
G: \mathbb{R} \times \mathbb{R}^{n} \rightarrow \mathbb{R}^{n},
$$

such that

$$
G(0,0)=0
$$

and

$$
\operatorname{det}\left[D_{x} G(0,0)\right] \neq 0 .
$$

Then there exists a unique differentiable function $X(\mu)$ defined on a neighborhood $M \subset \mathbb{R}$ of $\mu=0$,

$$
X: M \rightarrow \mathbb{R}^{n},
$$

such that $X(0)=0$ and

$$
G(\mu, X(\mu))=0, \mu \in M .
$$

In words the theorem says the following. Given $G(\mu, x)$ we assume that the zero set, i.e., the set of $(\mu, x)$ such that $G(\mu, x)=0$, contains at least one point $(0,0)$; see Fig. 7(a). If, in addition, the matrix

$$
\left(D_{x} G(0,0)\right)_{i j} \equiv \frac{\partial G_{i}}{\partial x_{j}}(0,0), i, j=1, \ldots, n
$$

has a nonzero determinant, then we can solve the equation $G(\mu, x)=0$ uniquely for $x$ as a function of $\mu$, at least for values of $\mu$ sufficiently near $\mu=0$. This means that, near $(\mu, x)=(0,0)$, the zero set of $G(\mu, x)$ consists of a single arc or branch as shown in Fig. 7(b).

\section{B. Applications to equilibria}

\section{Flows}

For Eqs. (1.2a) and (1.3a), we choose $G(\mu, x)=V(\mu, x)$. Then

$$
\operatorname{det}\left[D_{x} G(0,0)\right]=\operatorname{det}[D V(0,0)] ;
$$

this implies that condition (4.2b) will be satisfied if and only if $\lambda=0$ is not an eigenvalue for $D V(0,0)$. It then follows that small changes in $\mu$ will not destroy the equilibrium solution as long as zero is not an eigenvalue of the linear stability matrix for the equilibrium. The solution must persist and lie on a local branch of such solutions, $X(\mu)$, as required by the implicit function theorem.

Two further conclusions may be drawn. First, Hopf bifurcation cannot alter the number of equilibrium solutions, since the only eigenvalues of $D V$ on the imaginary
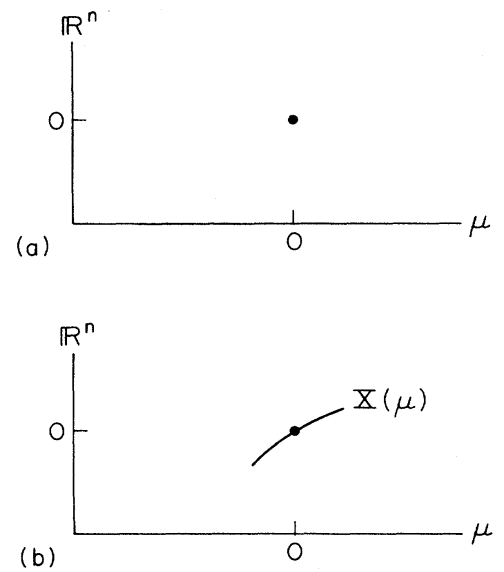

FIG. 7. A unique solution branch from the implicit function theorem. Given $G(0,0)=0$ and $\operatorname{det}\left[D_{x} G(0,0)\right] \neq 0$ at a point (a), the local structure of the solution set for $G(\mu, x)=0$ is a single branch (b). 
axis form a conjugate pair [Fig. 5(b)]. Second, the condition $\operatorname{det}[D V] \neq 0$ fails at a steady-state bifurcation, since by definition there is always an eigenvalue at zero. Thus in general we cannot expect a unique branch of equilibria through $(\mu, x)=(0,0)$ if this solution corresponds to a fixed point at criticality for steady-state bifurcation.

\section{Maps}

For Eqs. (1.2b) and (1.3b), we take $G(\mu, x)=f(\mu, x)$ $-x$, so that $G(0,0)=0$ and

$$
D_{x} G(0,0)=D f(0,0)-I,
$$

where $I$ is the identity matrix on $\mathbb{R}^{n}$. With this choice, if $G(\mu, x)=0$ then $x$ is a fixed point for the map at parameter value $\mu$. For the solution $(\mu, x)=(0,0)$, condition (4.2b) will be met if and only if the linear stability matrix $D f(0,0)$ does not have an eigenvalue at $\lambda=+1$. Provided $\lambda=1$ is not an eigenvalue, the implicit function theorem implies $(0,0)$ lies on an isolated branch of equilibrium solutions.

For the three basic instabilities illustrated in Fig. 6, only steady-state bifurcation involves an eigenvalue at +1 . Neither period-doubling nor Hopf bifurcation can alter the number of equilibrium solutions. In the context of Poincaré return maps for periodic orbits, these results on persistence of equilibria show that the periodic orbit can always be followed through a period-doubling or Hopf bifurcation. The question of following periodic orbits through parameter space in a global sense has also been studied (Mallet-Paret and Yorke, 1982; Yorke and Alligood, 1983).

\section{NORMAL-FORM DYNAMICS}

In this section we analyze very simple equations that describe the local dynamics associated with the linear instabilities of Sec. II. Remarkably, these simple examples are in fact quite general; to appreciate this generality requires the material on center manifolds and normal-form theory developed in later sections. Let us first analyze the dynamics of these simple models and then establish their generality. We shall consider the various bifurcations in the same order they were listed in Sec. II. In the following it is convenient to assume that criticality for an instability occurs at $\mu=0$.

\section{A. Flows}

1. Steady-state bifurcation: simple eigenvalue at zero

For a simple zero eigenvalue ${ }^{10}$ as illustrated in Fig. 5(a) the center-manifold reduction yields a system of the form

\footnotetext{
${ }^{10}$ An eigenvalue is simple if it is nondegenerate; for a real ei-
genvalue the associated eigenspace $(2.6 \mathrm{a})$ is then one dimension-

${ }^{10} \mathrm{An}$ eigenvalue is simple if it is nondegenerate; for a real ei-
genvalue the associated eigenspace $(2.6 \mathrm{a})$ is then one dimensional.
}

$$
\dot{x}=V(\mu, x), \quad x \in \mathbb{R}, \mu \in \mathbb{R},
$$

which will satisfy the following two conditions at criticality:

$$
\begin{aligned}
& V(0,0)=0, \\
& \frac{\partial V}{\partial x}(0,0)=0 .
\end{aligned}
$$

Center-manifold theory tells us that Eq. (5.1a) should be one dimensional. Furthermore, the reduction to one dimension will preserve Eq. (1.3a) and the occurrence of a zero eigenvalue; hence Eqs. (5.1b) and (5.1c), respectively. Expanding (5.1a) at $(\mu, x)=(0,0)$, we find

$$
\begin{aligned}
\dot{x}= & \frac{\partial V}{\partial \mu}(0,0) \mu+\frac{\partial^{2} V}{\partial x^{2}}(0,0) \frac{x^{2}}{2}+\frac{\partial^{2} V}{\partial \mu \partial x}(0,0) \mu x \\
& +\frac{\partial^{2} V}{\partial \mu^{2}}(0,0) \frac{\mu^{2}}{2}+\frac{\partial^{3} V}{\partial x^{3}}(0,0) \frac{x^{3}}{3 !}+\cdots,
\end{aligned}
$$

For this instability, the vector field at criticality,

$$
\dot{x}=\frac{\partial^{2} V}{\partial x^{2}}(0,0) \frac{x^{2}}{2}+\frac{\partial^{3} V}{\partial x^{3}}(0,0) \frac{x^{3}}{3 !}+\cdots,
$$

cannot be significantly simplified by making coordinate changes (cf. Sec. VIII); we shall obtain normal forms by making truncations and rescalings. There are three situations that arise most often in applications.

\section{a. Saddle-node bifurcation: the typical case}

Equations (5.1a)-(5.1c) define a steady-state bifurcation; without further assumptions we typically ("generically") expect

$$
\frac{\partial V}{\partial \mu}(0,0) \neq 0
$$

and

$$
\frac{\partial^{2} V}{\partial x^{2}}(0,0) \neq 0
$$

to hold. In this case Eq. (5.2) may be rewritten as

$\dot{x}=\frac{\partial V}{\partial \mu}(0,0) \mu[1+\mathcal{O}(\mu, x)]+\frac{\partial^{2} V}{\partial x^{2}}(0,0) \frac{x^{2}}{2}[1+\mathcal{O}(\mu, x)]$,

where $\mathcal{O}(\mu, x)$ indicates terms at least first order in $\mu$ or $x$. For example,

$$
\left[\frac{\partial^{2} V}{\partial \mu \partial x}(0,0) / \frac{\partial V}{\partial \mu}(0,0)\right] x,
$$

is one such term in the first bracket in Eq. (5.4). Near $(\mu, x) \approx(0,0)$ we can neglect these $\mathcal{O}(\mu, x)$ terms relative to unity and then define rescaled variables $(\widetilde{\mu}, \widetilde{x})$,

$$
\mu=\frac{2}{\left|\frac{\partial V}{\partial \mu}(0,0) \frac{\partial^{2} V}{\partial x^{2}}(0,0)\right|} \tilde{\mu}
$$




$$
x=\frac{2}{\left|\frac{\partial^{2} V}{\partial x^{2}}(0,0)\right|} \tilde{x},
$$

to obtain the normal form ${ }^{11}$

$$
\dot{\tilde{x}}=\epsilon_{1} \widetilde{\mu}+\epsilon_{2} \widetilde{x}^{2} \equiv \widetilde{V}(\widetilde{\mu}, \widetilde{x}),
$$

where

$$
\begin{aligned}
& \epsilon_{1}=\operatorname{sgn}\left[\frac{\partial V}{\partial \mu}(0,0)\right], \\
& \epsilon_{2}=\operatorname{sgn}\left[\frac{\partial^{2} V}{\partial x^{2}}(0,0)\right] .
\end{aligned}
$$

Obviously at $\widetilde{\mu}=0, \tilde{x}=0$ is an equilibrium in Eq. (5.6), and this equilibrium has a zero eigenvalue. What happens near $(\widetilde{\mu}, \widetilde{x})=(0,0)$ depends on $\left(\epsilon_{1}, \epsilon_{2}\right)$; there are four possibilities. Consider $\epsilon_{1}=\epsilon_{2}=+1$ (the other three cases can be analyzed similarly). Then the equilibria in Eq. (5.6) satisfy $\tilde{\mu}+\tilde{x}^{2}=0$. This describes a parabola in the $(\tilde{x}, \tilde{\mu})$ plane as shown in Fig. 8(a). At a fixed value of $\tilde{\mu}<0$, there are two equilibria $\tilde{x}_{ \pm}(\tilde{\mu})= \pm \sqrt{-\tilde{\mu}}$, which coalesce as $\tilde{\mu}$ increases to criticality. The upper branch $\tilde{x}_{+}(\tilde{\mu})$ is unstable, and the lower branch $\tilde{x}_{-}(\tilde{\mu})$ is asymptotically stable. This is indicated by the arrows in Fig. 8(a) and can be checked by linearizing Eq. (5.6) about $\tilde{x}_{ \pm}(\tilde{\mu})$. Let $\tilde{x}=\widetilde{x}_{ \pm}(\tilde{\mu})+\tilde{y}_{ \pm}$. Then (for $\left.\epsilon_{2}=+1\right)$

$$
\dot{\tilde{y}}_{ \pm}=\frac{\partial \widetilde{V}}{\partial \widetilde{x}}\left(\widetilde{\mu}, \widetilde{x}_{ \pm}\right) \widetilde{y}_{ \pm}=\left[2 \tilde{x}_{ \pm}(\widetilde{\mu})\right] \widetilde{y}_{ \pm} ;
$$

the eigenvalue $\left[2 \tilde{x}_{ \pm}(\tilde{\mu})\right]$ is positive (unstable) for $\tilde{x}_{+}$and negative (stable) for $\widetilde{x}_{-}$.

In two dimensions, a fixed point with one stable and one unstable eigenvector is referred to as a saddle; if the fixed point has two real negative eigenvalues it is a stable node (Arnold, 1973). When a parameter is varied, so that such fixed points are brought together, then the resulting merger can be described by the one-dimensional model (5.6); the bifurcation is named for this prototypical example. $^{12}$

Note that for $\tilde{\mu}<0$ there are two equilibria, but for $\tilde{\mu}>0$ there are none. This is consistent with the fact that Eq. $(4.2 b)$ fails at $(\mu, x)=(0,0)$ and the implicit function

\footnotetext{
${ }^{11}$ In the terminology of Sec. IV, the normal form is actually $\dot{\vec{x}}=\epsilon_{2} \widetilde{x}^{2}$, and $\epsilon_{1} \bar{\mu}$ is an unfolding term. I often overlook this distinction in the following and simply refer to the unfolded normal form as the normal form.

${ }^{12}$ More generally, the one-dimensional model (5.6) describes a much wider class of bifurcations, in which two fixed points are either created or destroyed. In higher dimensions it is not always the case that one equilibrium is stable and the other unstable; both may be unstable. Neither is it necessarily true that the eigenvalues not involved in the bifurcation must be real.
}
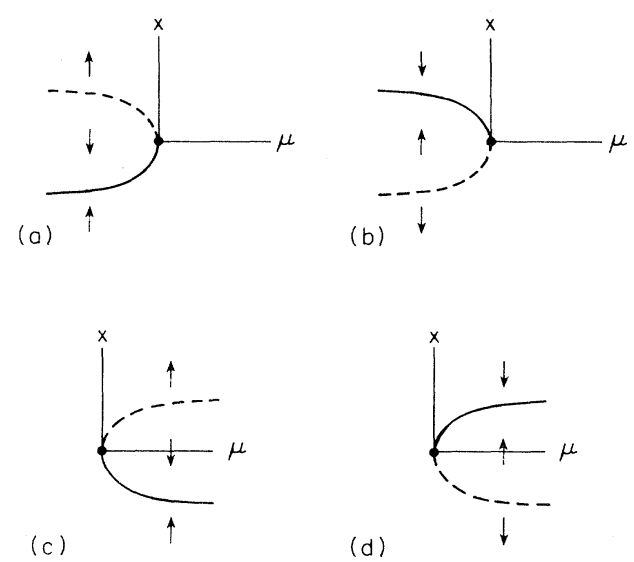

FIG. 8. Diagrams for saddle-node bifurcation with normal form (5.6): (a) $\epsilon_{1}=\epsilon_{2}=1$, (b) $\epsilon_{1}=\epsilon_{2}=-1$, (c) $-\epsilon_{1}=\epsilon_{2}=1$, (d) $\epsilon_{1}=-\epsilon_{2}=1$. Solid branches are stable; dashed branches are unstable.

theorem cannot guarantee a unique branch of equilibria passing through $(0,0)$.

The results for the remaining three cases, $\epsilon_{1}=-\epsilon_{2}=1$, $\epsilon_{1}=-\epsilon_{2}=-1$, and $\epsilon_{1}=\epsilon_{2}=-1$, are also shown in Fig. 8 . These diagrams in the $(\mu, x)$ plane are simple examples of bifurcation diagrams.

\section{b. Transcritical bifurcation: exchange of stability}

In applications, it may happen that an asymptotically stable equilibrium loses stability through a steady-state bifurcation, but the equilibrium solution itself survives. In this case saddle-node bifurcation, which characteristically destroys (or creates) equilibria, does not occur. When the equilibrium survives, we may denote it by $X(\mu)$ such that $X(0)=0$ and

$$
V(\mu, X(\mu))=0, \mu \in \mathbb{R}
$$

replaces Eq. (5.1b). Let us make the $\mu$-dependent change of variables $x=X(\mu)+x^{\prime}$ and then drop the primes. [This amounts to setting $X(\mu) \equiv 0$.] Then Eq. (5.8) becomes

$$
V(\mu, 0)=0,
$$

for an appropriately redefined $V(\mu, x)$. Since Eq. (5.9) implies

$$
\frac{\partial^{n} V}{\partial \mu^{n}}(0,0)=0, \quad n=1,2, \ldots,
$$

if we now make a Taylor expansion around $(\mu, x)=(0,0)$, then Eq. (5.2) is replaced by 
$\dot{x}=\frac{\partial^{2} V}{\partial \mu \partial x}(0,0) \mu x+\frac{\partial^{2} V}{\partial x^{2}}(0,0) \frac{x^{2}}{2}+\frac{\partial^{3} V}{\partial x^{3}}(0,0) \frac{x^{3}}{3 !}+\cdots$

Without further assumptions, we shall typically find

$$
\begin{aligned}
& \frac{\partial^{2} V}{\partial \mu \partial x}(0,0) \neq 0, \\
& \frac{\partial^{2} V}{\partial x^{2}}(0,0) \neq 0,
\end{aligned}
$$

where Eq. (5.12a) replaces (5.3a). Now, proceeding exactly as in the discussion of saddle-node bifurcation, we truncate and rescale variables to obtain a normal form,

$$
\dot{\tilde{x}}=\tilde{x}\left(\epsilon_{1} \tilde{\mu}+\epsilon_{2} \tilde{x}\right),
$$

where

$$
\epsilon_{1}=\operatorname{sgn}\left[\frac{\partial^{2} V}{\partial \mu \partial x}(0,0)\right]
$$

and

$$
\epsilon_{2}=\operatorname{sgn}\left[\frac{\partial^{2} V}{\partial x^{2}}(0,0)\right]
$$

Note that $\widetilde{x}=0$ is an equilibrium for all $\tilde{\mu}$, but at $\widetilde{\mu}=0$ the eigenvalue $\epsilon_{1} \widetilde{\mu}$ is zero. When $\operatorname{sgn}\left(\epsilon_{1} \tilde{\mu}\right)=-1(+1)$ the equilibrium $\tilde{x}=0$ is stable (unstable). The second factor on the right-hand side of Eq. (5.13) yields a second branch of equilibria, $\widetilde{x}_{b}(\tilde{\mu})$,

$$
\widetilde{x}_{b}(\widetilde{\mu})=-\left(\frac{\epsilon_{1}}{\epsilon_{2}}\right) \widetilde{\mu} .
$$

The stability of $\tilde{x}_{b}$ is found by linearizing Eq. (5.13) $\widetilde{x}=\widetilde{x}_{b}+y$ to find

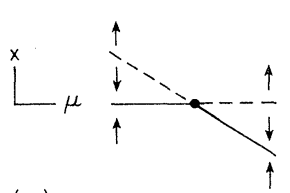

(a)

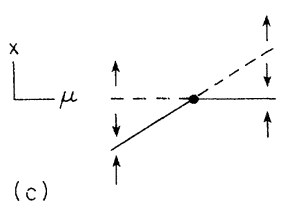

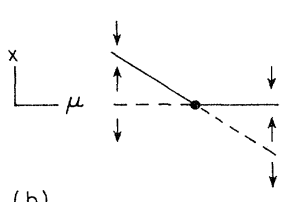

(b)

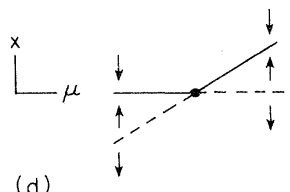

(d)

$$
\dot{y}=\left(-\epsilon_{1} \tilde{\mu}\right) y \text {. }
$$

Thus $\tilde{x}=0$ and $\tilde{x}=\tilde{x}_{b}(\tilde{\mu})$ have opposite stabilities; at $\tilde{\mu}=0$ these equilibria collide and their stabilities are "exchanged." The precise form of the resulting bifurcation diagram depends on $\epsilon_{1}$ and $\epsilon_{2}$; the four possibilities are shown in Fig. 9.

\section{c. Pitchfork bifurcation: reflection symmetry}

This version of steady-state bifurcation arises formally when Eq. (5.9) holds as in transcritical bifurcation but (5.12b) fails and is replaced by the assumption

$$
\frac{\partial^{3} V}{\partial x^{3}}(0,0) \neq 0
$$

A natural context for these assumptions is $V(\mu, x)$ having a reflection symmetry, i.e.,

$$
-V(\mu, x)=V(\mu,-x) \text {. }
$$

Obviously, this symmetry implies Eq. (5.9), and forces Eq. (5.12b) to fail. Replacing (5.12b) by (5.16), we may rewrite (5.11) as

$$
\begin{aligned}
\dot{x}= & \frac{\partial^{2} V}{\partial x \partial \mu}(0,0) \mu x[1+\mathcal{O}(\mu, x)] \\
& +\frac{\partial^{3} V}{\partial x^{3}}(0,0) \frac{x^{3}}{3 !}[1+\mathcal{O}(\mu, x)] .
\end{aligned}
$$

Now truncating higher-order terms and rescaling variables appropriately leads to the normal form

$$
\dot{\tilde{x}}=\widetilde{x}\left[\epsilon_{1} \tilde{\mu}+\epsilon_{2} \widetilde{x}^{2}\right],
$$

where

$$
\epsilon_{1}=\operatorname{sgn}\left[\frac{\partial^{2} V}{\partial \mu \partial x}(0,0)\right], \quad \epsilon_{2}=\operatorname{sgn}\left(\frac{\partial^{3} V}{\partial x^{3}}(0,0)\right] .
$$

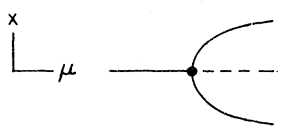

(a)

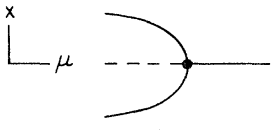

(c)

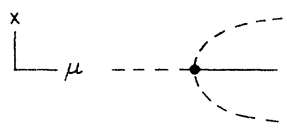

(b)

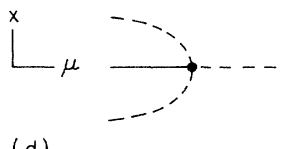

(d)
FIG. 9. Diagrams for transcritical bifurcation with normal form (5.13): (a) $\epsilon_{1}=\epsilon_{2}=1$, (b) $\epsilon_{1}=\epsilon_{2}=-1$, (c) $-\epsilon_{1}=\epsilon_{2}=1$, (d) $\epsilon_{1}=-\epsilon_{2}=1$. Solid branches are stable; dashed branches are unstable.
FIG. 10. Diagrams for pitchfork bifurcation with normal form (5.19): (a) $\epsilon_{1}=-\epsilon_{2}=1$, (b) $-\epsilon_{1}=\epsilon_{2}=1$, (c) $\epsilon_{1}=\epsilon_{2}=-1$, (d) $\epsilon_{1}=\epsilon_{2}=1$. solid branches are stable; dashed branches are unstable. 


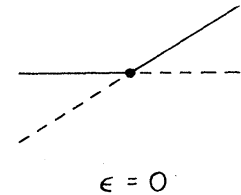

(a)

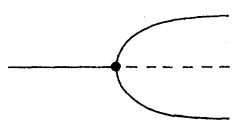

$\epsilon=0$

(b)
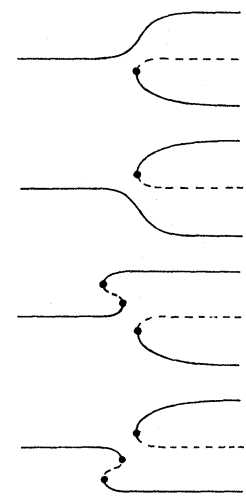

$\epsilon \neq 0$
FIG. 11. Perturbing nongeneric diagrams: (a) transcritical bifurcation; (b) pitchfork bifurcation.

The analysis of Eq. (5.19) differs from transcritical in that the second factor in (5.19) contributes two branches of equilibria,

$$
\widetilde{x}_{ \pm}(\mu)= \pm \sqrt{-\left(\epsilon_{1} / \epsilon_{2}\right) \tilde{\mu}},
$$

which only exist for $\operatorname{sgn}\left(\epsilon_{1} \tilde{\mu} / \epsilon_{2}\right)=-1$. The stability of the solutions may be worked out as before, and the four possibilities are illustrated in Fig. 10. The bifurcation diagrams resemble pitchforks in the $(\tilde{\mu}, \tilde{x})$ plane, hence the name.

We conclude this discussion of steady-state bifurcation by indicating how perturbations of transcritical or pitchfork bifurcation can restore the expected "generic" behavior, i.e., saddle-node bifurcation. ${ }^{13}$ Suppose $V(\mu, x)$ describes a transcritical or pitchfork bifurcation at $(\mu, x)=(0,0)$. We can perturb $V(\mu, x)$ by including a small term $V_{i}(\mu, x)$ in the dynamics,

$$
\dot{x}=V(\mu, x)+\epsilon V_{1}(\mu, x),
$$

where $0 \leq \epsilon<1$. The perturbation $V_{1}$ may be chosen ar-

\footnotetext{
${ }^{13}$ In the presence of such perturbations the transcritical or pitchfork bifurcation is said to be imperfect. A rigorous and systematic theory of such imperfect bifurcations can be developed using the techniques of singularity theory (Golubitsky and Schaeffer, 1985).
}

bitrarily in the sense that it need not respect any special assumptions such as Eqs. (5.17), (5.9), or (5.1b). For transcritical bifurcation, when $\epsilon \neq 0$ one expects the bifurcation diagram to be modified in one of two ways [see Fig. 11(a)]. In one case the perturbed diagram contains two saddle-node bifurcations; in the other case there are no bifurcations at all. With pitchfork bifurcation there are four possibilities expected for the perturbed diagram, Fig. 11(b). There is one important new feature: the possibility of finding hysteresis in the bifurcations of the perturbed pitchfork. This effect can be understood intuitively by noting that when $\epsilon=0$ the outer branches of the pitchfork meet the middle branch with an angle of exactly $90^{\circ}$. A small perturbation will split and join the branches as shown and also perturb this $90^{\circ}$ angle slightly. This latter effect leads to the appearance of hysteresis.

\section{Hopf bifurcation: a single conjugate pair of imaginary eigenvalues}

The normal form is two dimensional and in polar coordinates $(r, \theta)$ may be written as

$\dot{r}=r\left[\gamma(\mu)+\sum_{j=1}^{\infty} a_{j}(\mu) r^{2 j}\right]=\gamma r+a_{1} r^{3}+\mathcal{O}\left(r^{5}\right)$,

$\dot{\theta}=\omega(\mu)+\sum_{j=1}^{\infty} b_{j}(\mu) r^{2 j}$,

where $\gamma(\mu) \pm i \omega(\mu)$ is the complex-conjugate pair of eigenvalues that are assumed to satisfy

$$
\begin{aligned}
& \gamma(0)=0, \omega(0) \neq 0, \\
& \frac{d \gamma}{d \mu}(0)>0 .
\end{aligned}
$$

The conditions (5.23) simply mean that the conjugate pair crosses the imaginary axis at $\mu=0$ in a nondegenerate way.

A characteristic feature of Eq. (5.22) is the absence of $\theta$ on the right-hand side. This means that the dynamics of the normal form is invariant with respect to the group of rotations of the phase $\theta$. In the literature, this invariance is called the $S^{1}$ phase-shift symmetry, ${ }^{14}$ and it allows the dynamics of Eq. (5.22a) to be analyzed independently from (5.22b).

For $(5.22 \mathrm{a})$, we assume that at criticality $(\mu=0)$ the cubic coefficient does not vanish,

$$
a_{1}(0) \neq 0
$$

\footnotetext{
${ }^{14}$ The phase shifts in $\theta$ are described mathematically by the rotation group $S O(2)$ or, equivalently, as the action of the circle group $S^{1}$. It is conventional to use the latter terminology for the Hopf normal-form symmetry.
} 
then the solutions to $d r / d t=0$ near $r=0$ are determined by the sign of $a_{1}(0)$ (see Fig. 12). Consider $a_{1}(0)<0$ for example - from Eq. (5.22a) the radial equilibria satisfy

$$
r\left(\gamma(\mu)+a_{1}(\mu) r^{2}\right) \approx 0
$$

and there are two branches: $r=0$ and $r_{H}(\mu) \approx \sqrt{-\gamma / a_{1}}$. The latter solution exists only for $\gamma(\mu)>0$ since $r_{H}$ must be real. When Eq. (5.22b) is taken into account we see that this new solution in fact describes a periodic orbit of amplitude $r_{H}$ and frequency $\omega_{H} \approx \omega(\mu)+\sum_{j=1}^{\infty} b_{j}(\mu) r_{H}^{2}$. The plot of $\dot{r}$ vs $r$ in Fig. 12(a) makes it clear that the periodic orbit is asymptotically stable; this can be checked analytically by linearizing (5.22a) about $r=r_{H}$ and determining the linear eigenvalue. The bifurcation diagram is also drawn in Fig. 12(a); since the new branch of solutions is found in the direction of increasing $\mu$, above the threshold for instability of the equilibrium, the bifurcation of $r_{H}$ is said to be supercritical.

The analysis for $a_{1}(0)>0$ is similar but the results are slightly different. Now the $r_{H}$ solution is found only for $\gamma(\mu)<0$ or $\mu<0$. In this case the branch of periodic solutions is subcritical and unstable ${ }^{15}$; see Fig. 12(b).

Hopf bifurcation is a richer phenomenon than steadystate bifurcation in the sense that it leads to timedependent nonlinear behavior. In an experiment, a supercritical Hopf bifurcation manifests itself in the spontaneous onset of oscillatory behavior. Often this oscillation corresponds to the appearance of a wave in the system.

\section{B. Maps}

1. Steady-state bifurcation: simple eigenvalue at +1

The normal form is one dimensional,

$$
x_{j+1}=f\left(\mu, x_{j}\right), \quad \mu \in \mathbb{R}, x \in \mathbb{R},
$$

for $j=0,1,2, \ldots$, where

$$
\begin{aligned}
& f(0,0)=0, \\
& \frac{\partial f}{\partial x}(0,0)=+1 .
\end{aligned}
$$

Let $V(\mu, x) \equiv f(\mu, x)-x$. Then to find fixed points for $f$ we need to solve

$$
V(\mu, x)=0 .
$$

Note that

\footnotetext{
${ }^{15}$ There is no consensus in the literature as to how the terms supercritical and subcritical should be defined in general, although all conventions agree with my usage in this context. For a didactic discussion advocating one sensible set of definitions see Tuckerman and Barkley (1990).
}

$$
V(0,0)=0
$$

and

$$
\frac{\partial V}{\partial x}(0,0)=0
$$

follow from (5.26b) and (5.26c), respectively. This problem corresponds to finding the branches of equilibria in a steady-state bifurcation for flows, i.e., Eqs. (5.27) are equivalent to Eqs. (5.1). Consequently, insofar as the branches of equilibria are concerned, we have precisely the cases already studied.
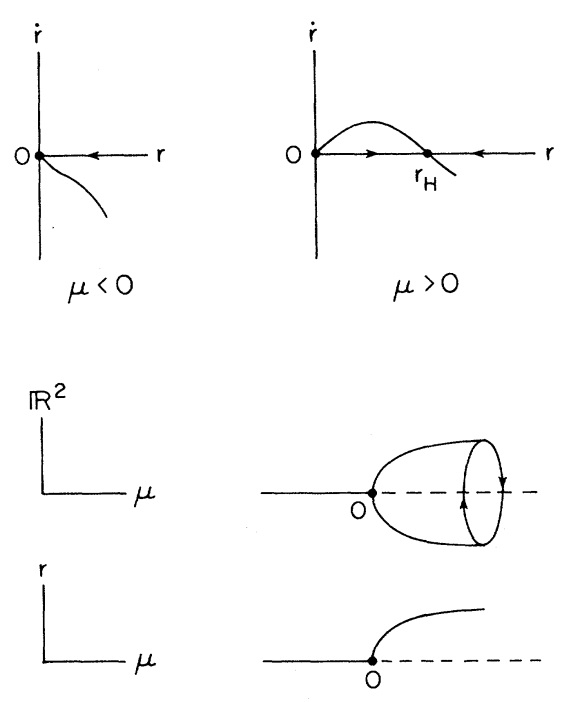

(a)
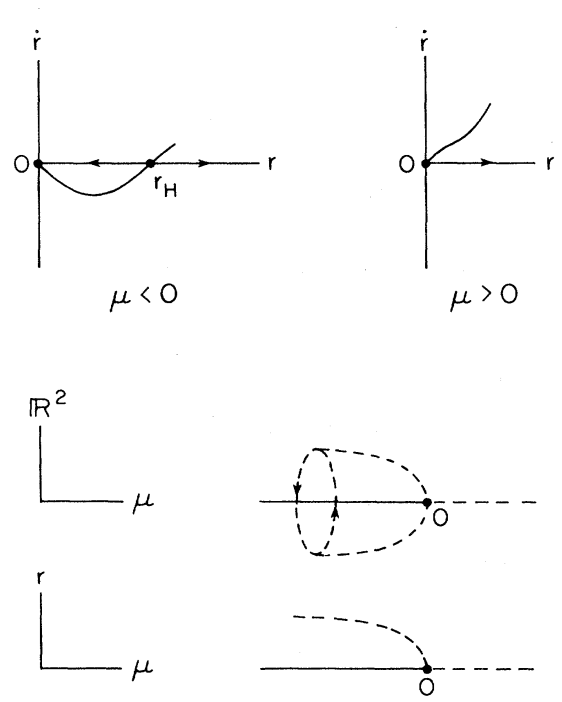

(b)

FIG. 12. Radial dynamics and diagrams for Hopf bifurcation with normal form (5.22): (a) supercritical bifurcation $a_{1}(0)<0$; (b) subcritical bifurcation $a_{1}(0)>0$. 


\section{a. Saddle-node bifurcation}

As before, this occurs if

$$
\frac{\partial V}{\partial \mu}(0,0) \neq 0 \text { and } \frac{\partial^{2} V}{\partial x^{2}}(0,0) \neq 0 \text {. }
$$

From Eq. (5.28) and our previous discussion of saddlenode bifurcation for flows, we are led to the normal form

$$
\widetilde{x}_{j+1}=\epsilon_{1} \tilde{\mu}+\widetilde{x}_{j}+\epsilon_{2} \widetilde{x}_{j}^{2} \equiv \widetilde{f}\left(\widetilde{\mu}, \widetilde{x}_{j}\right)
$$

for this bifurcation in the rescaled variables (5.5) with

$$
\epsilon_{1}=\operatorname{sgn}\left[\frac{\partial f}{\partial \mu}(0,0)\right], \quad \epsilon_{2}=\operatorname{sgn}\left[\frac{\partial^{2} f}{\partial x^{2}}(0,0)\right] .
$$

Since the analysis of branches of fixed points for Eq. (5.29) is equivalent to finding equilibria for Eq. (5.6), we need only check the stability of $\tilde{x}_{ \pm}(\tilde{\mu})= \pm \sqrt{-\tilde{\mu}}$. The linear eigenvalue at $\widetilde{x}_{ \pm}$is simply

$$
\frac{\partial \widetilde{f}}{\partial \tilde{x}}\left(\tilde{\mu}, \widetilde{x}_{ \pm}\right)=1+2 \epsilon_{2} \widetilde{x}_{ \pm}(\tilde{\mu})
$$

from Eq. (5.29), hence $\widetilde{x}_{ \pm}(\tilde{\mu})$ is stable (unstable) if $\epsilon_{2} \widetilde{x}_{ \pm}(\tilde{\mu})$ is negative (positive). Thus the stability assignments for the branches of equilibria turn out to be the same as in the bifurcation diagrams for flows (see Fig. 8).

The interpretation of these diagrams depends on how we interpret the map. If we imagine that the saddle-node bifurcation occurs in a Poincaré return map for a periodic orbit in a flow, then the branches of solutions diagrammed in Fig. 8 correspond to mergers of periodic orbits.

\section{b. Transcritical bifurcation}

This bifurcation occurs if Eq. (5.28) is replaced by

$$
\frac{\partial V}{\partial \mu}(0,0)=0
$$

and

$$
\frac{\partial^{2} V}{\partial x \partial \mu}(0,0) \neq 0, \quad \frac{\partial^{2} V}{\partial x^{2}}(0,0) \neq 0 .
$$

From our previous discussion of the normal form (5.13) for flows, we obtain

$$
\widetilde{x}_{j+1}=\widetilde{x}_{j}\left(1+\epsilon_{1} \tilde{\mu}+\epsilon_{2} \widetilde{x}_{j}\right) \equiv \widetilde{f}\left(\widetilde{\mu}, \widetilde{x}_{j}\right)
$$

as the normal form in this case. The bifurcation diagrams for the branches of fixed points are shown in Fig. 9, and the stability assignments in Fig. 9 are also correct, since the linear eigenvalues for $\tilde{x}=0$ and $\tilde{x}=\widetilde{x}_{b}$ in Eq. (5.32) are $\left(1+\epsilon_{1} \tilde{\mu}\right)$ and $\left(1-\epsilon_{1} \tilde{\mu}\right)$, respectively. At $\widetilde{\mu}=0$ the two branches of fixed points merge and exchange stability.

\section{c. Pitchfork bifurcation}

This case occurs if Eq. (5.31a) holds while (5.31b) is replaced by

$$
\frac{\partial^{2} V}{\partial x^{2}}(0,0)=0
$$

and

$$
\frac{\partial^{2} V}{\partial \mu \partial x}(0,0) \neq 0, \frac{\partial^{3} V}{\partial x^{3}}(0,0) \neq 0
$$

From Eq. (5.19) we obtain the normal form,

$$
\widetilde{x}_{j+1}=\tilde{x}\left(1+\epsilon_{1} \tilde{\mu}+\epsilon_{2} \tilde{x}_{j}^{2}\right) \text {. }
$$

The analysis of the branches of fixed points and their stabilities yield the same bifurcation diagrams as in the pitchfork bifurcation for flows (Fig. 10).

\section{Period-doubling bifurcation:} a simple eigenvalue at -1

In Sec. IV we proved that this instability does not change the number of fixed-point solutions, thus any branches of solutions bifurcating from the equilibrium will necessarily have different dynamical properties. The normal form is one dimensional and has a reflection symmetry,

$$
\begin{aligned}
& x_{j+1}=f\left(\mu, x_{j}\right), \mu \in \mathbb{R}, x \in \mathbb{R}, \\
& f(\mu, 0)=0, \\
& \frac{\partial f}{\partial x}(0,0)=-1, \\
& -f(\mu, x)=f(\mu,-x) .
\end{aligned}
$$

In writing Eq. (5.35b), we have made use of the fact that the branch of fixed points $X(\mu)$ through $(\mu, x)=(0,0)$ must persist and have assumed a coordinate shift which places the branch at the origin. With these properties, the Taylor expansion of $f(\mu, x)$ at the fixed point $x=0$ takes the form

$f(\mu, x)=\lambda(\mu) x+\alpha_{1}(\mu) x^{3}+\alpha_{2}(\mu) x^{5}+\mathcal{O}\left(x^{7}\right)$,

where $\lambda(0)=-1$. The trick is to notice that the twiceiterated map, $f^{2}(\mu, x) \equiv f(\mu, f(\mu, x))$, is undergoing a steady-state bifurcation, which is a pitchfork because of the reflection symmetry (5.35d) of the normal form. Following our discussion of pitchfork bifurcation, we take $V(\mu, x)=f^{2}(\mu, x)-x$ and check the prerequisite conditions (5.31a), (5.33) using (5.35) and (5.36):

$$
\begin{aligned}
& \frac{\partial V}{\partial \mu}(0,0)=\frac{\partial f}{\partial \mu}(0,0)\left[1+\frac{\partial f}{\partial x}(0,0)\right]=0, \\
& \frac{\partial^{2} V}{\partial x^{2}}(0,0)=\frac{\partial^{2} f}{\partial x^{2}}(0,0) \frac{\partial f}{\partial x}(0,0)\left[1+\frac{\partial f}{\partial x}(0,0)\right]=0
\end{aligned}
$$

and

$$
\frac{\partial^{2} V}{\partial \mu \partial x}(0,0)=2 \frac{\partial f}{\partial x}(0,0) \frac{\partial^{2} f}{\partial \mu \partial x}(0,0)=-2 \frac{d \lambda}{d \mu}(0),
$$




$$
\begin{aligned}
\frac{\partial^{3} V}{\partial x^{3}}(0,0) & =\frac{\partial f}{\partial x}(0,0) \frac{\partial^{3} f}{\partial x^{3}}(0,0)\left[1+\left[\frac{\partial f}{\partial x}(0,0)\right]^{2}\right] \\
& =-12 \alpha_{1}(0)
\end{aligned}
$$

respectively. Thus to satisfy Eq. (5.33) we need only assume $(d \lambda / d \mu)(0) \neq 0$ and $\alpha_{1}(0) \neq 0$ in Eqs. (5.37c) and (5.37d); each of these two conditions is compatible with Eqs. (5.35) and will typically be satisfied. The normal form for the pitchfork in $f^{2}(\mu, x)$ is

$$
\widetilde{x}_{j+1}=\widetilde{x}_{j}\left(1+\epsilon_{1} \widetilde{\mu}+\epsilon_{2} \widetilde{x}_{j}^{2}\right) \text {, }
$$

where

$$
\epsilon_{1}=\operatorname{sgn}\left(-\frac{d \lambda}{d \mu}(0)\right], \quad \epsilon_{2}=\operatorname{sgn}\left(-\alpha_{1}(0)\right),
$$

with the bifurcation diagrams for fixed points of $f^{2}(\mu, x)$ shown in Fig. 10.

These diagrams for $f^{2}(\mu, x)$ show three branches of fixed points $x=0$ and $x=\tilde{x}_{ \pm}(\tilde{\mu})$, and we now consider the implications for the original map $f(\mu, x)$. Obviously, the $x=0$ branch is the fixed point for $f(\mu, x)$, whose stability is lost at $\mu=0$. The $\tilde{x}_{ \pm}(\tilde{\mu})$ branches of the pitchfork for $f^{2}(\mu, x)$ cannot be fixed points for $f(\mu, x)$, since the implicit function theorem guarantees that $x=0$ is the unique branch through $(\mu, x)=(0,0)$. Therefore $\left(\widetilde{x}_{+}, \widetilde{x}_{-}\right)$must represent a new bifurcating branch of two-cycles for $f(\mu, x)$. More precisely, denoting $\tilde{x}_{ \pm}$as $x_{ \pm}$in the original variables of Eq. (5.35), we must have

$$
\begin{aligned}
& x_{-}=f\left(\mu, x_{+}\right), \\
& x_{+}=f\left(\mu, x_{-}\right) .
\end{aligned}
$$

The conclusion that $f(\mu, x)$ must interchange $x_{+}$and

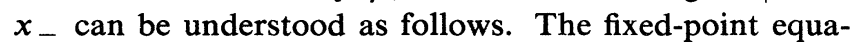
tion $f^{2}\left(\mu, x_{+}\right)=x_{+}$implies that the image of $x_{+}$, $x^{\prime}+=f\left(\mu, x_{+}\right)$, will also be a nonzero fixed point for $f^{2}(\mu, x)$, i..e, $x_{+}^{\prime}=f^{2}\left(\mu, x_{+}^{\prime}\right)$. But we know that the pitchfork bifurcation for $f^{2}$ produces only two nonzero branches of fixed points, so $x_{+}^{\prime}$ must coincide with $x_{-}$; hence Eq. (5.38a) follows. Moreover, the reflection symmetry of $f(\mu, x)$ requires $x_{-}=-x_{+}$when the dynamics is represented by the normal form. ${ }^{16}$ The stability of the two-cycle $\left(x_{+}, x_{-}\right)$is determined by the stability of $x_{+}$ (or $x_{-}$) as fixed points for $f^{2}$ and is correctly indicated in Fig. 10.

If we consider the bifurcation from the perspective that Eq. (5.35) describes an instability of a fixed point in the return map for a periodic orbit, then the bifurcating two-cycle represents a bifurcating branch of periodic or-

\footnotetext{
${ }^{16}$ In fact, the reflection symmetry of the period-doubling normal form implies that all new branches of two-cycles can be calculated by solving $f(\mu, x)=-x$; it is not necessary to consider explicitly the second iterate of $f$ (cf. Crawford, Knobloch, and Riecke 1990).
}

bits with approximately twice the period of the original orbit (see Fig. 13). This leads to the terminology perioddoubling bifurcation.

\section{Hopf bifurcation: simple complex-conjugate pair at $|\lambda|=1$}

The normal form in this case is two dimensional; however, its structure depends on subtleties not evident in the examples of steady-state bifurcation or period-doubling bifurcations. Denote the complex eigenvalue by

$$
\lambda(\mu)=(1+a(\mu)) e^{i 2 \pi \theta(1+b(\mu))},
$$

where

$$
\begin{aligned}
& 0<\theta<\frac{1}{2}, \\
& a(0)=b(0)=0 \text { and } \frac{d a}{d \mu}(0)>0 .
\end{aligned}
$$

If the eigenvalue at criticality $\lambda(0)=e^{i 2 \pi \theta}$ satisfies the nonresonance conditions,

$$
\lambda(0)^{3} \neq 1 \text { and } \lambda(0)^{4} \neq 1,
$$

then in polar variables $(r, \psi)$ the normal form for the bifurcation is

$$
\begin{aligned}
& r_{j+1}=(1+a(\mu)) r_{j}\left[1+a_{1}(\mu) r_{j}^{2}+\mathcal{O}\left(r_{j}^{4}\right)\right], \\
& \psi_{j+1}=\psi_{j}+2 \pi \theta(1+b(\mu))+b_{1}(\mu) r_{j}^{2}+\mathcal{O}\left(r_{j}^{4}\right) .
\end{aligned}
$$

At this order in $r_{j}$, the right-hand side is independent of $\psi$, a feature analogous to the phase-shift symmetry encountered in the normal form for Hopf bifurcation in a flow. In Sec. VIII, we shall show that this $\psi$ independence depends on the nonresonance conditions (5.40). If these conditions are relaxed then $\psi$-dependent terms will appear in Eq. (5.41); when Eq. (5.40) holds, the dependence on $\psi$ will first occur in terms that are indicated as $\mathcal{O}\left(r^{4}\right)$ in Eq. (5.41).

For small $r$, we neglect the higher-order terms in Eq. (5.41) and then solve the radial dynamics separately from the phase evolution. For this tactic to succeed, the cubic term in Eq. (5.41a) must not vanish at criticality, i.e., we require

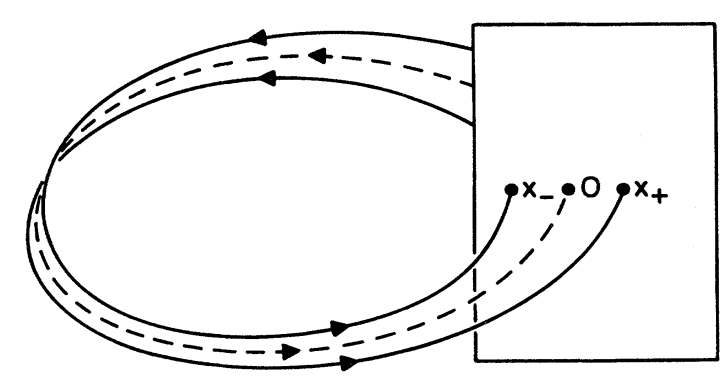

FIG. 13. Period-doubling bifurcation in a Poincaré return map. 


$$
a_{1}(0) \neq 0
$$

then Eq. (5.41a) describes a pitchfork bifurcation at $\mu=0$. Only the positive bifurcating branch

$$
r_{H}=\left(-\frac{a}{a_{1}}\right)^{1 / 2}
$$

is relevant, since $r$ must be non-negative. In combination with Eq. (5.41b) the $r_{H}$ branch describes a circle of radius $r_{H}$ that is mapped into itself by (5.41), i.e., the circle is invariant under iteration of the dynamics (5.41).

This branch of invariant circles may be either supercritical or subcritical depending on the sign of $a / a_{1}$ in Eq. (5.43). With the eigenvalue in (5.39a) assumed to be leaving the unit circle $(5.39 \mathrm{c})$, we have

$$
\begin{aligned}
\operatorname{sgn}\left(\frac{a}{a_{1}}\right) & =\operatorname{sgn}\left(\frac{\mu \frac{d a}{d \mu}(0)}{a_{1}(0)}+\mathcal{O}\left(\mu^{2}\right)\right) \\
& =\operatorname{sgn}\left(\mu a_{1}(0)\right)
\end{aligned}
$$

near $\mu=0$. Therefore, if $a_{1}(0)<0$, the invariant circle is found when $\mu>0$ ( supercritical), and if $a_{1}(0)>0$, then the branch bifurcates when $\mu<0$ (subcritical). Using Eq. (5.41a), one can show that the supercritical branch is stable and the subcritical branch will be unstable. Furthermore, one can prove that these invariant circles persist and have the properties just described if the $\mathcal{O}\left(r^{4}\right)$ terms in Eq. (5.41a) are restored (Ruelle and Takens, 1971; Lanford, 1973). However Eq. (5.41b) is much less satisfactory as a description of the dynamics on the invariant circle. According to $(5.41 \mathrm{~b})$, the circle dynamics is simply a fixed rotation by

$$
\Delta \psi \approx 2 \pi(\theta+b(\mu))+b_{1}(\mu) r_{H}^{2}+\mathcal{O}\left(r_{H}^{4}\right) .
$$

In the theory of maps of the circle (Guckenheimer and Holmes, 1986; Arnold, 1988a), it is well known that such a uniform rotation is unstable if subjected to small perturbations. Indeed, with the inclusion of small $\psi$ dependent perturbations present in the $\mathcal{O}\left(r^{4}\right)$ terms of Eq. (5.41b), we expect phenomena such as mode locking to occur in the dynamics on the circle; see Rasband

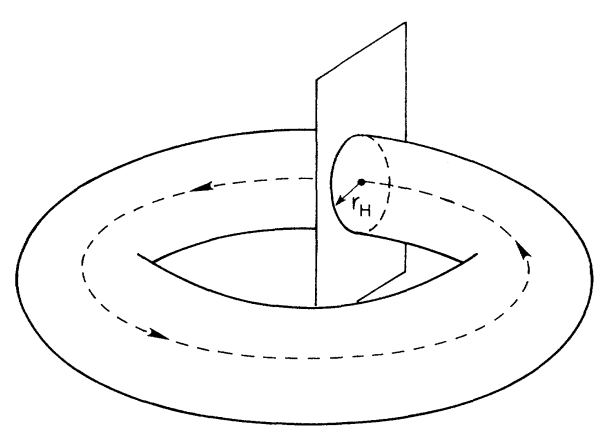

FIG. 14. Hopf bifurcation in a Poincaré return map.
(1990) for an introductory discussion.

Finally, we consider this bifurcation from the perspective that Eq. (5.41) describes an instability of a periodic orbit as viewed in the return map to a Poincare section. In this setting the invariant circle that appears in the section corresponds to a two-dimensional invariant torus in the flow; see Fig. 14.

\section{Final remarks}

If normal forms are to be generally useful, we must show that the bifurcation analysis of an arbitrary highdimensional system can be reduced to a simple normal form. It is not obvious that we should be able to get as much information in one or two dimensions as we can in several, nor is it obvious that we will be able, even in low dimensions, to find coordinates in which our dynamical system is so simple.

The reduction in dimensionality is accomplished by observing that the interesting dynamics near a bifurcation occurs on a low-dimensional subset of phase space called the center manifold. ${ }^{17}$ The dimension of this center manifold determines the dimension of the normal form. The simple structure of the normal form is established by the theory of Poincaré-Birkhoff normal forms.

\section{INVARIANT MANIFOLDS FOR EQUILIBRIA}

A mathematically precise definition of manifolds and related geometric ideas may be found in many places, for example Chillingworth (1976), or Guillemin and Pollack (1974). Intuitively, a $d$-dimensional manifold in $\mathbb{R}^{n}$ should be visualized as a smooth surface forming a subset of $\mathbb{R}^{n}$. For example, a closed loop in $\mathbb{R}^{2}$ and the surface of a doughnut in $\mathbb{R}^{3}$ are one- and two-dimensional manifolds, respectively.

Suppose $M$ denotes a manifold in the phase space $\mathbb{R}^{n}$ of a dynamical system Eq. (1.2a) or (1.2b). Let $m \in M$ be an arbitrary point on the manifold, and let $\mathcal{O}_{m}$ denote the trajectory of the dynamical system through $m$, i.e., $x(0)=m$ for Eq. (1.2a) and $x_{j=0}=m$ for Eq. (1.2b). If $\mathcal{O}_{m} \subset M$ for all $m \in M$, then $M$ is an invariant manifold for the dynamical system. More concisely, an invariant manifold is a surface that is carried into itself by the dynamics.

If $M \subset \mathbb{R}^{n}$ is an invariant manifold, then the full $\mathrm{dy}$ namics on $\mathbb{R}^{n}$ implies the existence of a distinct autonomous dynamical system defined on $M$ alone, which can

\footnotetext{
${ }^{17}$ Liapunov-Schmidt reduction is an alternative procedure for reducing the dimension of the problem. An introduction to this technique may be found in Golubitsky and Schaeffer (1985); the connection between center-manifold reduction and LiapunovSchmidt reduction has been explored by Chossat and Golubitsky (1987) and Marsden (1979).
} 
in principle be studied independently. For example, if a map (1.2b) admits an invariant circle, then the dynamics on this circle is described by a one-dimensional map of the circle to itself, e.g.,

$$
\theta_{j+1}=f\left(\theta_{j}\right) \bmod (2 \pi),
$$

where the angle $\theta$ labels points on the circle. The invariance of the circle implies that $f(\theta)$ will not depend on the other phase-space coordinates. Thus Eq. (6.1) describes an autonomous one-dimensional dynamical system embedded in the dynamics $(1.2 b)$ on a larger phase space.

Individual trajectories provide very simple examples of invariant manifolds. In a flow, an equilibrium and a periodic orbit are invariant manifolds with zero and one dimension, respectively. Much less trivial examples are the stable, center, and unstable manifolds associated with equilibria. ${ }^{18}$ We first consider flows; the manifolds for maps are quite similar and they are discussed briefly in subsection VI.B.

\section{A. Flows}

For a flow (1.2a), (1.3a),

$$
\dot{x}=V(\mu, x) \text {, }
$$

the stable, center, and unstable manifolds for an equilibrium are generalizations of the invariant linear subspaces $E^{s}, E^{c}$, and $E^{u}$ that arise in the linearized dynamics

$$
\dot{x}=D V(0,0) \cdot x \text {. }
$$

These subspaces were described in Sec. II.A [cf. Eq. (2.7)]; hereafter we denote their dimensions by $n_{s}, n_{c}$, and $n_{u}$, respectively.

For the linear system (6.3), the subspaces (2.7) are in fact invariant manifolds. However, they are atypical, since these manifolds are also linear vector spaces; this special additional property reflects the linearity of $\mathrm{Eq}$. (6.3). When the nonlinear terms in Eq. (6.2) are restored, the invariant manifolds just constructed for the linear system are perturbed but they persist. Their qualitative features also persist, except that the vector-space structure is lost. Intuitively, the nonlinear effects deform the invariant linear vector spaces into invariant nonlinear manifolds.

For an equilibrium $x=0$, we have the following definition. A stable manifold is an invariant manifold of dimension $n_{s}$ that contains $x=0$ and is tangent to $E^{s}$ at

\footnotetext{
${ }^{18}$ There is an extensive mathematical theory of invariant manifolds with application to sets far more complex than the equilibria considered here. For a relatively introductory discussion see Irwin (1980) and Lanford (1983); other standard mathematical references include Hirsch, Pugh, and Shub (1977) and Shub (1987).
}

$x=0$. The unstable and center manifolds may be similarly defined by replacing $E^{s}$ with $E^{u}$ and $E^{c}$, respectively. We shall denote these manifolds by $W^{s}, W^{u}$, and $W^{c}$, see Fig. 2(c).

The stable and unstable manifolds are unique. Furthermore, trajectories in these manifolds have some simple dynamical properties. If $x(t) \in W^{s}$, then $x(t) \rightarrow 0$ as $t \rightarrow+\infty$; if $x(t) \in W^{u}$, then $x(t) \rightarrow 0$ as $t \rightarrow-\infty$. This asymptotic behavior is indicated schematically in Fig. 2(c).

The properties of center manifolds are somewhat more subtle (Lanford, 1973; Carr, 1981; Sijbrand, 1985). In general, the center manifold is not unique; we give an example of this nonuniqueness below. There is no general characterization of the dynamics on $W^{c}$, not even asymptotically as $|t| \rightarrow \infty$. Nevertheless center manifolds play a distinguished role in bifurcation theory because of two important properties. We discuss these properties here, and in Sec. VII we state a generalization of the Hartman-Grobman theorem that justifies our discussion.

For a center manifold $W^{c}$, there exists a neighborhood $U$ of $x=0$ such that

(i) if $x(0) \in U$ has forward trajectory $x(t)$ in $U$, i.e., $x(t) \in U$ for all $t \geq 0$, then as $t \rightarrow \infty$ the trajectory $x(t)$ converges to $W^{c}$;

(ii) if $x(0) \in U$ has a trajectory in $U$, i.e., $x(t) \in U$ for $-\infty<t<\infty$, then $x(0) \in W^{c}$ and by invariance the entire trajectory must lie in $W^{c}$.

One does not know in general how large $U$ will be, only that such a neighborhood exists; the situation is illustrated in Fig. 15.

The first property (i) is sometimes referred to as local attractivity. Notice that there is no claim here that a typi-

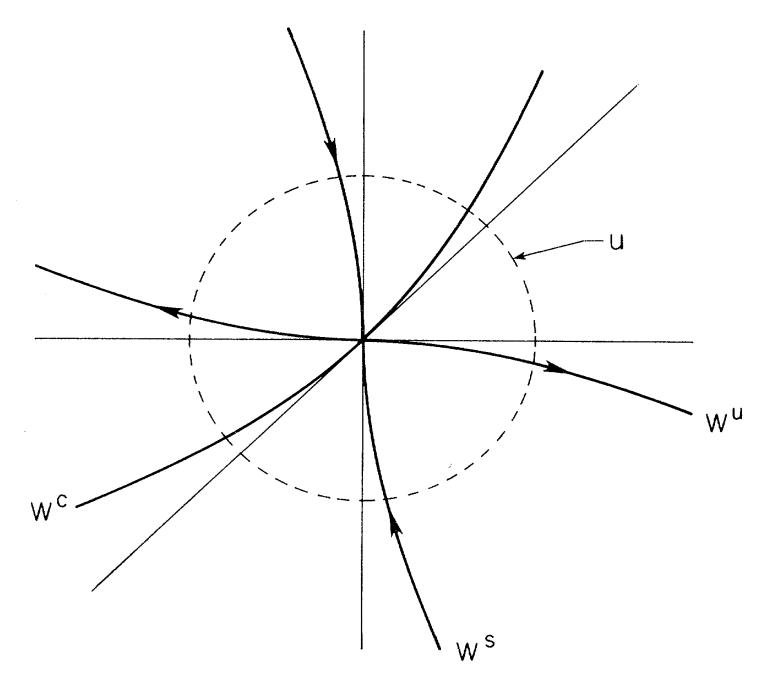

FIG. 15. Neighborhood $U$ within which $W^{c}$ is locally attracting. 
cal initial condition will satisfy the required hypothesis; in particular, if there is an unstable manifold then most points will be pushed away from $W^{c}$. Local attractivity holds only for points $x(0) \in U$ whose orbits remain sufficiently close to $x=0$ for all future times.

The second property is a special case of the first and provides sufficient conditions for a trajectory to lie in $W^{c}$. In particular, property (ii) implies that invariant sets of any type, e.g., equilibria, periodic orbits, invariant 2-tori, must lie in $W^{c}$ if they are contained in $U$. For this reason one may restrict attention to the flow on $W^{c}$ when analyzing a local bifurcation; this restriction provides a setting of lower dimension with no loss of generality. We return to this point in Sec. VII.

There is an interesting way to reformulate property (ii) so that it refers only to the forward trajectory. A point $x(0)$ is recurrent if, for any $T>0$ and any $\epsilon>0$, there exists a time $t_{0}>T$ such that $\left|x\left(t_{0}\right)-x(0)\right|<\varepsilon$. In other words, the recurrent trajectory returns arbitrarily close to $x(0)$ over and over again-forever:

(ii)' if $x(0) \in U$ is recurrent and the forward trajectory $x(t)$ is contained in $U$, then local attractivity [property (i)] implies $x(0) \in W^{c}$.

Thus one can say that the center manifold captures all local recurrence. ${ }^{19}$

\section{B. Maps}

The invariant manifolds for an equilibrium (1.3b) of a map (1.2b) may be described in very similar terms. We indicate only the necessary modifications in the discussion for flows.

The linearized map for Eq. (1.2b),

$$
x_{j+1}=D f(0,0) \cdot x_{j},
$$

determines invariant linear subspaces $E^{s}, E^{c}, E^{u}$ that were described in Sec. II.B. One defines the stable $\left(W^{s}\right)$, center $\left(W^{c}\right)$, and unstable $\left(W^{u}\right)$ manifolds relative to these subspaces just as for flows. The manifold $W^{\alpha}(\alpha=s, c, u)$ is an invariant manifold of dimension $n_{\alpha}$ which is tangent to $E^{\alpha}$ at $x=0$.

In addition, the discussion of the properties of these manifolds for flows applies to the case of maps as well, with the obvious modification of replacing continuous time by discrete iteration.

\footnotetext{
${ }^{19}$ Dynamical systems theory utilizes various notions of recurrent behavior. In addition to the recurrent points, there is the larger set of nonwandering points. A point $x(0)$ is a wandering point if there exists some neighborhood $V$ of $x(0)$ such that for $t$ sufficiently large the trajectory $x(t)$ never reenters $V$. A point that is not a wandering point is a nonwandering point; all recurrent points are nonwandering. The local nonwandering points in the neighborhood $U$ are in the center manifold.
}

\section{CENTER-MANIFOLD REDUCTION}

For the various bifurcations introduced in Sec. II, the goal is to detect and analyze new branches of solutions, e.g., fixed points and periodic orbits. This analysis should determine their existence, their dynamics, and their stability. It is important to note that these branches emerge from the given equilibrium in a continuous fashion as $\mu$ varies near zero. For $\mu$ sufficiently small, the distance from the original equilibrium to the new solution can be made arbitrarily small. Therefore these small-amplitude (recurrent) solutions will fall within the neighborhood of local attractivity for $W^{c}$; hence they are contained in the center manifold. This conclusion is correct, but the argument just given ignores a subtlety: the bifurcation analysis requires that we work on an interval in parameter space about $\mu=0$, but our locally attracting center manifold is defined at only a single point $\mu=0$ when the system is critical. (Indeed, for saddlenode bifurcation one does not even have an equilibrium when $\mu$ is slightly supercritical.) This awkward discrepancy can be finessed by formally applying centermanifold reduction to the "suspended system" for Eqs. (1.2a) and (1.2b). This extension is described in Sec. VII.C below, and it establishes the existence of a locally attracting submanifold on a full neighborhood of $\mu=0$.

For the moment we shall accept the conclusion that all continuously bifurcating branches of solutions will lie in an appropriately defined center manifold. Since the center manifold is invariant, the dynamics on the manifold is autonomous. That is, one has an independent dynamical system of dimension $\operatorname{dim} W^{c}=n_{c}$, which describes exactly the trajectories of points on $W^{c}$. In particular, this reduced dynamical system describes all local bifurcations in $W^{c}$. Our goal is to derive the equations for this reduced dynamical system, at least approximately.

\section{A. Flows}

In general, the nonlinearity of a center manifold prevents us from obtaining an exact analytic description of its dynamics. However, near the equilibrium $x=0$, it is possible to accomplish this task with sufficient accuracy to obtain useful results.

At criticality $(\mu=0)$ for an instability, the spectrum of $D V(0,0)$ is contained in the left half-plane $(\operatorname{Re} \lambda<0)$ except for the critical modes whose eigenvalues satisfy $\operatorname{Re} \lambda=0$. Our method of deriving the center-manifold dynamics does not require the absence of unstable modes, however, and we shall describe the procedure without assuming $E^{u}$ is empty. Thus consider $D V(0,0)$ with a spectrum like that illustrated in Fig. 2(a), and write Eq. (1.2a) as

$$
\frac{d x}{d t}=D V(0,0) \cdot x+N(x)
$$

for $\mu=0$, where $N(x)$ denotes the nonlinear terms. 
Without loss of generality we can choose variables $x_{1} \in E^{c}, x_{2} \in E^{s} \oplus E^{u}$, such that $x=\left(x_{1}, x_{2}\right)$ and Eq. (7.1) becomes

$$
\begin{aligned}
& \frac{d}{d t} x_{1}=A \cdot x_{1}+N_{1}\left(x_{1}, x_{2}\right), \\
& \frac{d}{d t} x_{2}=B \cdot x_{2}+N_{2}\left(x_{1}, x_{2}\right),
\end{aligned}
$$

where $A$ is an $n_{c} \times n_{c}$ matrix with all eigenvalues on the imaginary axis, $B$ is an $\left(n_{s}+n_{u}\right) \times\left(n_{s}+n_{u}\right)$ matrix with all eigenvalues off the imaginary axis, and $N_{1}, N_{2}$ are the resulting nonlinear terms in $\left(x_{1}, x_{2}\right)$ variables

$$
N_{1}: \mathbb{R}^{n} \rightarrow E^{c}, \quad N_{2}: \mathbb{R}^{n} \rightarrow E^{s} \oplus E^{u} .
$$

\section{Local representation of $W^{c}$}

A center manifold associated with $E^{c}$ will pass through $x=0$, and at $x=0$ the manifold will be tangent to $E^{c}$. This tangency means that near $x=0$ one can describe $W^{c}$ as the graph of a function $h\left(x_{1}\right)$,

$$
h: E^{c} \rightarrow E^{u} \oplus E^{s}, \quad h\left(x_{1}\right)=x_{2},
$$

where for $x_{1}$ sufficiently small the point $x=\left(x_{1}, h\left(x_{1}\right)\right)$ belongs to $W^{c}$. Since $x=0$ is in the center manifold, we require

$$
h(0)=0,
$$

and the tangency condition at $x=0$ implies $^{20}$

$$
D_{x_{1}} h(0)=0 \text {. }
$$

The geometric interpretation of this representation is illustrated in Fig. 16 for the particular three-dimensional example $n_{s}=1, n_{c}=2$, and $E^{u}$ empty.

The invariance of $W^{c}$ implies an equation for $h\left(x_{1}\right)$. Let $x^{c}(t)=\left(x_{1}^{c}(t), x_{2}^{c}(t)\right)$ denote a trajectory of Eq. (7.1) that belongs to $W^{c}$ and has sufficiently small amplitude that we may write

$$
x_{2}^{c}(t)=h\left(x_{1}^{c}(t)\right) .
$$

This implies immediately that

$$
\begin{aligned}
\frac{d x_{2}^{c}}{d t} & =D h\left(x_{1}^{c}(t)\right) \cdot \frac{d x_{1}^{c}}{d t} \\
& =D h\left(x_{1}^{c}(t)\right) \cdot\left[A \cdot x_{1}^{c}+N_{1}\left(x_{1}^{c}, h\left(x_{1}^{c}\right)\right)\right]
\end{aligned}
$$

from Eqs. (7.2a) and (7.4). However, (7.2b) provides a

\footnotetext{
${ }^{20}$ This follows from the observation that tangent vectors to $W^{c}$ at $(0,0)$ must have the form $\left(x_{1}, 0\right)$. Let $s(\epsilon)=\left(x_{1}(\epsilon), x_{2}(\epsilon)\right)$ denote an arc lying in the center manifold and passing through $(0,0)$ when $\epsilon=0$. Then for small $\epsilon, x_{2}(\epsilon)$ $=h\left(x_{1}(\epsilon)\right)$, and the tangent vector $\dot{s}(0)$ can be written $\dot{s}(0)=\left(\dot{x}_{1}(0), D_{x_{1}} h(0) \cdot \dot{x}_{1}(0)\right)$; hence $D_{x_{1}} h(0) \cdot \dot{x}_{1}(0)=0$. Since $\dot{x}_{1}(0)$ is arbitrary, we must require Eq. (7.3c).
}
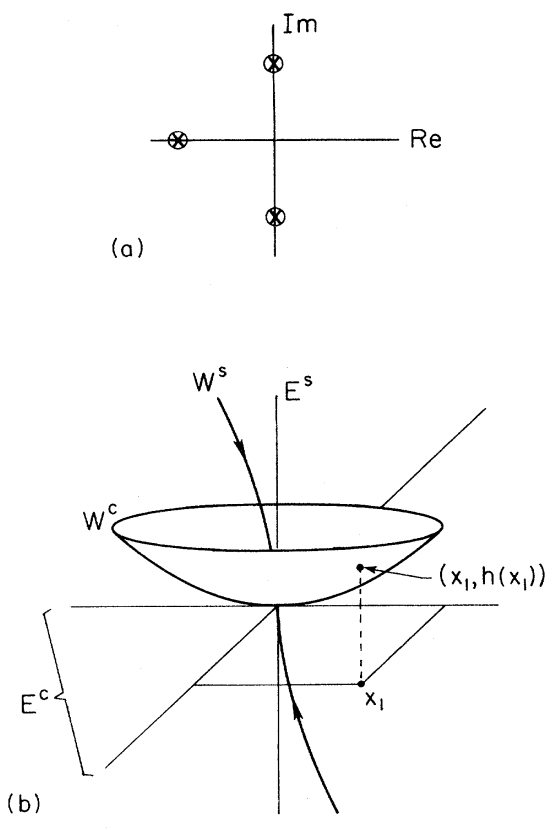

FIG. 16. Geometry of the graph representation. When there are no unstable modes, a center manifold $W^{c}$ may be represented as the graph of a map $h\left(x_{1}\right)$ from $E^{c}$ to $E^{s}$. For the linear spectrum shown in (a) we have the situation illustrated in (b) where $\operatorname{dim} E^{c}=2$ and $\operatorname{dim} E^{s}=1$.

second expression for $\dot{x}_{2}^{c}$,

$$
\frac{d x_{2}^{c}}{d t}=B \cdot h\left(x_{1}^{c}\right)+N_{2}\left(x_{1}^{c}, h\left(x_{1}^{c}\right)\right),
$$

which must be the same as Eq. (7.5) if the trajectory remains on $W^{c}$. Combining Eqs. (7.5) and (7.6) yields the desired equation for $h\left(x_{1}\right)$ :

$$
\begin{aligned}
D h\left(x_{1}\right) \cdot\left[A \cdot x_{1}+N_{1}\left(x_{1}, h\left(x_{1}\right)\right)\right] & \\
& =B \cdot h\left(x_{1}\right)+N_{2}\left(x_{1}, h\left(x_{1}\right)\right) .
\end{aligned}
$$

A solution to this equation that also satisfies Eqs. (7.3b) and (7.3c) determines a center manifold near $x=0$.

The dynamics on the center manifold $h\left(x_{1}\right)$ follows from Eqs. (7.2a) and (7.4):

$$
\frac{d}{d t} x_{1}=A \cdot x_{1}+N_{1}\left(x_{1}, h\left(x_{1}\right)\right) \text {. }
$$

By replacing $x_{2}$ with $h\left(x_{1}\right)$ in Eq. (7.2a) we have decoupled (7.2a) from (7.2b); thus Eq. (7.8) describes an autonomous flow in $n_{c}$ dimensions. These two results, (7.7) and (7.8), are the crucial (exact) equations required to reduce a bifurcation problem to the center manifold.

The "invariance equation" (7.7) is in general a nonlinear partial differential equation for $h\left(x_{1}\right)$ and cannot be solved in closed form except in special cases. However, we can solve Eq. (7.7) approximately by representing $h\left(x_{1}\right)$ as a formal power series, 


$$
\begin{aligned}
\phi\left(x_{1}\right)= & \sum_{i, j=1}^{n_{c}} \phi_{i j}\left(x_{1}\right)_{i}\left(x_{1}\right)_{j} \\
& +\sum_{i, j, k=1}^{n_{c}} \phi_{i j k}\left(x_{1}\right)_{i}\left(x_{1}\right)_{j}\left(x_{1}\right)_{k}+\cdots
\end{aligned}
$$

where $\left(x_{1}\right)_{i}$ denotes the $i$ th component of $x_{1}$ and the coefficients $\phi_{i j}, \phi_{i j k}$, etc. are $\left(n_{s}+n_{u}\right)$-dimensional column vectors. It can be shown that if $\phi\left(x_{1}\right)$ satisfies $\phi(0)=0, D_{x_{1}} \phi(0)=0$ and solves Eq. (7.7) to $\mathcal{O}\left(x_{1}^{p}\right)$, i.e.,

$$
\begin{aligned}
D \phi\left(x_{1}\right) \cdot\left[A \cdot x_{1}+N_{1}\left(x_{1}, \phi\left(x_{1}\right)\right)\right] \\
=B \cdot \phi\left(x_{1}\right)+N_{2}\left(x_{1}, \phi\left(x_{1}\right)\right)+\mathcal{O}\left(x_{1}^{p}\right),
\end{aligned}
$$

then

$$
h\left(x_{1}\right)=\phi\left(x_{1}\right)+\mathcal{O}\left(x_{1}^{p}\right) \text { as } x_{1} \rightarrow 0
$$

(Carr, 1981). It is a straightforward calculation to insert $\phi\left(x_{1}\right)$ from Eq. (7.9) into (7.7) and solve for the coefficients to any desired order. Examples of this calculation are provided in Sec. VII.A. 3 and Sec. IX.

\section{The Shoshitaishvili theorem}

In Sec. II, the Hartman-Grobman theorem for hyperbolic equilibria demonstrated that local bifurcations required a loss of hyperbolicity. In the present notation, the theorem applies when there are no eigenvalues on the imaginary axis and Eq. (7.2a) is absent; then the flow of Eq. (7.2b) can be mapped onto the flow of

$$
\frac{d x_{2}}{d t}=B \cdot x_{2}
$$

on a neighborhood of $x_{2}=0$.

For a nonhyperbolic equilibrium, the theorem was generalized by Shoshitaishvili to allow for the effect of the critical modes (7.2a); in effect, Eq. (7.12) must be supplemented by the center-manifold dynamics (7.8).

Theorem VII.1. Let $\phi_{t}(x)$ denote the flow of Eq. (7.1) and $\widetilde{\phi}_{t}\left(x_{1}, x_{2}\right)$ denote the flow for the decoupled system

$$
\frac{d x_{1}}{d t}=A \cdot x_{1}+N_{1}\left(x_{1}, h\left(x_{1}\right)\right), \frac{d x_{2}}{d t}=B \cdot x_{2} \text {. }
$$

Then there exists a homeomorphism ${ }^{6}$

$$
\Psi: \mathbb{R}^{n} \rightarrow \mathbb{R}^{n}
$$

and a neighborhood $U$ of $x=0$ where

$$
\phi_{t}(x)=\Psi^{-1} \circ \widetilde{\phi}_{t} \circ \Psi(x)
$$

for all $(x, t)$ such that $x \in U$ and $\phi_{t}(x) \in U$.

This result was proved by Pliss (1964) in the circumstance that there are no unstable modes $\left(n_{u}=0\right)$. Shoshitaishvili $(1972,1975)$ generalized Pliss's result to allow for both unstable modes and dependence on param-

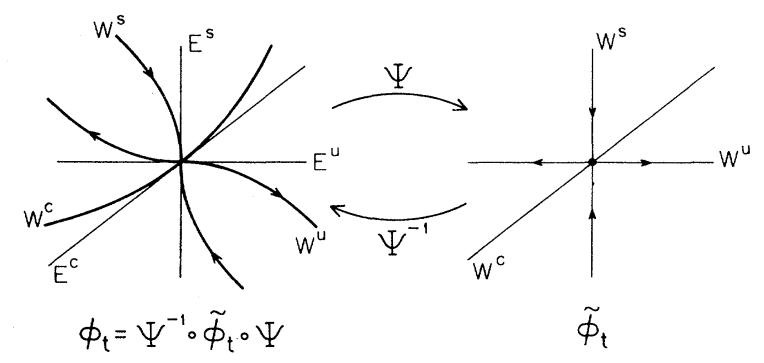

FIG. 17. Geometric illustration of Shoshitaishvili's theorem. The local change of coordinates $\Psi$ in Shoshitaishvili's theorem maps the flow $\phi_{t}$ of the original system onto a simpler flow $\widetilde{\phi}_{t}$ for which nonlinear effects are confined to the dynamics on the center manifold.

eters. This theorem is also discussed in Arnold (1988a) and Vanderbauwhede (1989).

Heuristically, the change of coordinates $\Psi$ "straightens out" the nonlinear manifolds of Eq. (7.1) locally; note that for Eq. (7.13) the invariant manifolds coincide with the linear invariant subspaces (see Fig. 17). In addition, the flow $\widetilde{\phi}_{t}$ transverse to the center manifold is linear.

A useful feature of this theorem is the information it provides on the stability of solutions in the center manifold. The first equation in (7.13) describes stability relative to perturbations within the center manifold, and the second equation characterizes stability relative to perturbations transverse to the center manifold. Thus for the original nonlinear problem (7.1) the stability to transverse perturbations can be inferred from the eigenvalues of the matrix $B$ in Eq. (7.13).

The properties (i) and (ii) of center manifolds discussed in Sec. VI.A follow from the equivalence in Eq. (7.15). Consider the decoupled system (7.13) and suppose $x(0) \in U$ is an initial condition whose forward trajectory $x(t)$ remains in $U$. Since $x(0)=\left[x_{1}(0), x_{2}(0)\right]$, there are two cases: if $x_{2}(0) \neq 0$ then $x(0)$ must lie in the stable manifold, otherwise the component in the unstable manifold would grow without bound, forcing $x(t)$ to leave $U$; if $x_{2}(0)=0$ then $x(0) \in W^{c}$. In either case, the forward trajectory will converge to $W^{c}$ as $t \rightarrow \infty$. For the second property, we assume that the entire trajectory $x(t)$ remains in $U$ for $-\infty<t<\infty$. Now if $x_{2}(0) \neq 0$ there must be components of $x(0)$ in either $W^{s}$ or $W^{u}$ (or both), which grow without bound as $|t| \rightarrow \infty$. Therefore the assumption $x(t) \in U$ for all $t$ requires $x_{2}(0)=0$, which implies $x(0) \in W^{c}$ for Eq. (7.13). Because of $\Psi$, these properties for (7.13) will also hold for the center manifold of (7.15) described locally by $h\left(x_{1}\right)$.

\section{Example}

Consider the two-dimensional flow

$$
\left[\begin{array}{l}
\dot{x} \\
\dot{y}
\end{array}\right]=\left(\begin{array}{cc}
0 & 0 \\
0 & -1
\end{array}\right)\left(\begin{array}{l}
x \\
y
\end{array}\right)+\left(\begin{array}{c}
-x^{3} \\
x^{2}
\end{array}\right)
$$


whose equilibrium $(x, y)=(0,0)$ determines $E^{s}$ and $E^{c}$ as

$$
\begin{aligned}
& E^{s}=\{(x, y) \mid x=0\}, \\
& E^{c}=\{(x, y) \mid y=0\} .
\end{aligned}
$$

Note that in this example the stable manifold $W^{s}$ coincides with $E^{s}$ because $\dot{x}$ does not depend on $y$. The center manifold has a graph representation $y=h(x)$ near $(x, y)=(0,0)$, and the invariance equation (7.7) for this example is the ordinary differential equation

$$
\frac{d h}{d x}\left[-x^{3}\right]=-h(x)+x^{2} .
$$

We first calculate the asymptotic description $\phi(x)$ as in Eqs. (7.9) and (7.10),

$$
\phi(x)=\phi_{2} x^{2}+\phi_{3} x^{3}+\phi_{4} x^{4}+\cdots,
$$

and obtain $\phi_{2}=1, \phi_{3}=0, \phi_{4}=2$, so that

$$
h(x)=x^{2}+2 x^{4}+\mathcal{O}\left(x^{5}\right) .
$$

It turns out that in this example Eq. (7.18) can be solved exactly by the method of variation of parameters. Dropping $x^{2}$ in (7.18), we obtain the solution to the homogeneous problem

$$
h_{0}(x)=c_{1} e^{-1 / 2 x^{2}} .
$$

Then setting $h(x)=A(x) h_{0}(x)$ in (7.18) yields

$$
\frac{d A}{d x}=-\frac{e^{1 / 2 x^{2}}}{c_{1} x},
$$

with the solution

$$
c_{1} A(x)=c+\frac{1}{2} \int_{1}^{1 / x^{2}} \frac{e^{y / 2}}{y} d y .
$$

Hence the solution to Eq. (7.18) is

$$
h(x)=e^{-1 / 2 x^{2}}\left[c+\frac{1}{2} \int_{1}^{1 / x^{2}} \frac{e^{y / 2}}{y} d y\right] .
$$

The prefactor $e^{-1 / 2 x^{2}}$ enforces $h(x) \rightarrow 0$ as $x \rightarrow 0$ and $h^{\prime}(x) \rightarrow 0$ as $x \rightarrow 0$. Note that Eq. (7.23) contains an arbitrary constant. Hence the solution is not unique, and in fact the equilibrium $(x, y)=(0,0)$ has an uncountably infinite number of distinct center manifolds. However, the term $c e^{-1 / 2 x^{2}}$ causing the lack of uniqueness vanishes to all orders at the origin, so these manifolds all have the same power-series representation (7.19). One can show that this circumstance is generally the case (Sijbrand, 1985): when the center manifold is not unique the differences are too small to be detected in the asymptotic description (7.9). Thus in practice one does not worry about possible non-uniqueness, since it will not affect practical calculations based on the power-series representation of the center manifold.

Finally, for this example the center-manifold dynamics $(7.8)$ is

$$
\dot{x}=-x^{3}
$$

there is no dependence on $h(x)$ because $\dot{x}$ in Eq. (7.12) is independent of $y$.

\section{B. Maps; Local representation of $W^{c}$}

The reduction procedure for a map is wholly analogous to that just described for flows. With the splitting of $x=(y, z)$ where $y \in E^{c}$ and $z \in E^{s} \oplus E^{u}$, the dynamics (1.2b) becomes

$$
\begin{aligned}
& y_{j+1}=A \cdot y_{j}+Y\left(y_{j}, z_{j}\right), \\
& z_{j+1}=B \cdot z_{j}+Z\left(y_{j}, z_{j}\right)
\end{aligned}
$$

in a manner equivalent to Eq. (7.2).

A center manifold for $(y, z)=(0,0)$ may be locally represented by a graph $z=h(y)$ as in Eqs. (7.3a), (7.3b), and $(7.3 \mathrm{c})$. The invariance of $W^{c}$ implies that $h(y)$ must satisfy

$$
h(A \cdot y+Y(y, h(y)))=B \cdot h(y)+Z(y, h(y))
$$

by the same reasoning used before. Combining the solution to Eq. (7.25) with (7.24a) yields

$$
y_{j+1}=A \cdot y_{j}+Y\left(y_{j}, h\left(y_{j}\right)\right),
$$

which describes the dynamics on $W^{c}$ near $y=0$. In practice, the solution to Eq. (7.25) is obtained approximately using power series (7.9) as before.

\section{Working on intervals in parameter space: suspended systems}

Let $\mu=0$ be the critical parameter value for an equilibrium undergoing either steady-state or Hopf bifurcation. At $\mu=0$, there is a locally attracting center manifold $W^{c}$ that contains all small-amplitude equilibria and periodic orbits; these solutions can be detected by analyzing a low-dimensional system on $W^{c}$. Unfortunately, the small-amplitude solutions of interest do not usually exist at $\mu=0$; or rather, they have "zero amplitude" at criticality. These new bifurcating solutions become distinct from the original equilibrium only for nonzero $\mu$, and when $\mu \neq 0$ we have no center subspace $E^{c}$ and thus no center manifold to justify studying the reduced dynamical system Eq. (7.8) or Eq. (7.26) [see Fig. 18(a)].

Ruelle and Takens (1971) pointed out that the reduction was justified not only at $\mu=0$, but in fact on a neighborhood of criticality $\mu \in\left(-\mu_{0}, \mu_{0}\right)$, in parameter space. Indeed, the notion of locally attractivity defined in Sec. V implies the existence of such a neighborhood; the procedure of Ruelle and Takens is to apply center-manifold reduction to the "suspended system." This trick works equally well for flows and maps; we consider only the argument for flows.

It is convenient to split the variables in Eq. (1.2a) as was done in subsection VII.A above. Let $\mathbb{R}^{n}=E^{c} \oplus X$ where $E^{c}$ is the center subspace associated with the bifurcation at $\mu=0$ and $X$ is the subspace spanned by the 
remaining eigenvectors. We choose variables $x=\left(x_{1}, x_{2}\right)$ such that $x_{1} \in E^{c}$ and $x_{2} \in X$; then Eq. (1.2a) becomes

$$
\begin{aligned}
& \frac{d x_{1}}{d t}=V_{i}\left(\mu, x_{1}, x_{2}\right), \\
& \frac{d x_{2}}{d t}=V_{2}\left(\mu, x_{1}, x_{2}\right) .
\end{aligned}
$$

At criticality, this splitting coincides with Eq. (7.2):

$$
\begin{aligned}
& V_{1}(0,0,0)=V_{2}(0,0,0)=0, \\
& D_{x_{2}} V_{1}(0,0,0)=D_{x_{1}} V_{2}(0,0,0)=0, \\
& D_{x_{1}} V_{1}(0,0,0)=A, \\
& D_{x_{2}} V_{2}(0,0,0)=B .
\end{aligned}
$$

Instead of applying center-manifold reduction to Eq. (7.27) at $\mu=0$, we form the suspended system for (7.27):

$$
\begin{aligned}
& \frac{d x_{1}}{d t}=V_{1}\left(\mu, x_{1}, x_{2}\right), \\
& \frac{d x_{2}}{d t}=V_{2}\left(\mu, x_{1}, x_{2}\right), \quad\left(\mu, x_{1}, x_{2}\right) \in \mathbb{R} \times \mathbb{R}^{n},
\end{aligned}
$$

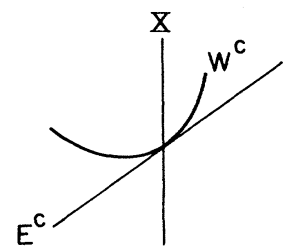

(a)

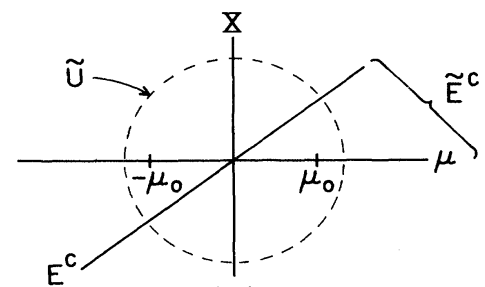

(b)

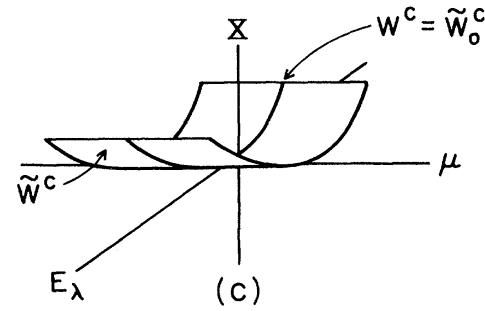

FIG. 18. Illustration of the suspended system: (a) the center manifold for the original system; (b) the enlarged center subspace $\widetilde{E}^{c}$ and a neighborhood of local attractivity $\widetilde{U}$ for $\widetilde{W}^{c}$ ( $\widetilde{W}^{c}$ is not shown); (c) schematic appearance of $\widetilde{W}^{c}$ when the fixed point is not destroyed by the bifurcation. The original center manifold $W^{c}$ is recovered by slicing $\widetilde{W}^{c}$ at $\mu=0$. $\frac{d \mu}{d t}=0$

and formally apply center-manifold theory to the equilibrium $\left(\mu, x_{1}, x_{2}\right)=(0,0,0)$ of Eq. (7.27'). Since Eqs. (7.27) and $\left(7.27^{\prime}\right)$ are obviously equivalent, the only virtue of this exercise is that certain features of Eqs. (7.27) are made explicit. Note that when Eq. $\left(7.27 c^{\prime}\right)$ is appended, the linear spectrum of Eqs. $\left(7.27^{\prime}\right)$ at $\mu=0$ now includes a zero eigenvalue not found in the spectrum of Eqs. (7.27) at $\mu=0$; hence the center subspace $\widetilde{E}^{c}$ for $\left(7.27^{\prime}\right)$ at $\left(\mu, x_{1}, x_{2}\right)=(0,0,0)$ is larger than $E^{c}$ for Eqs. (7.27), and similarly the center manifold $\widetilde{W}^{c}$ for Eqs. $\left(7.27^{\prime}\right)$ is larger than the center manifold $W^{c}$ in (7.27) for $\left(x_{1}, x_{2}\right)=(0,0)$ at $\mu=0$. More precisely, we have $\widetilde{W}^{c} \supset W^{c}$ and $\operatorname{dim} \widetilde{W}^{c}=\operatorname{dim} W^{c}+1$. Since $\left(\mu, x_{1}\right)$ provide coordinates on $\widetilde{E}^{c}$, we can describe $\widetilde{W}^{c}$ as a graph: $x_{2}=\widetilde{h}\left(\mu, x_{1}\right)$, where $\widetilde{h}$ satisfies

$$
D_{x_{1}} \tilde{h}\left(\mu, x_{1}\right) \cdot V_{1}\left(\mu, x_{1}, \tilde{h}\right)=V_{2}\left(\mu, x_{1}, \widetilde{h}\right)
$$

subject to

$$
\begin{aligned}
& \widetilde{h}(0,0)=0, \\
& D_{x_{1}} \tilde{h}(0,0)=0, \\
& \frac{\partial \widetilde{h}}{\partial \mu}(0,0)=0 .
\end{aligned}
$$

The crucial observation is that local recurrent points belong to $\widetilde{W}^{c}$ for $\mu$ near 0 (rather than simply at $\mu=0$ ). Let $\widetilde{U} \subset \mathbb{R} \times \mathbb{R}^{n}$ denote a neighborhood of $\left(\mu, x_{1}, x_{2}\right)=(0,0,0)$ within which $\widetilde{W}^{c}$ is locally attracting; the intersection of $\widetilde{U}$ with the $\mu$ axis defines an open set containing $\mu=0$. Within this open set we can find a value of $\mu$, denoted $\mu_{0}$, such that the interval $\left(-\mu_{0}, \mu_{0}\right)$ on the $\mu$ axis is contained in $\widetilde{U}$ [see Fig. 18(b)]. When $\mu^{\prime} \in\left(-\mu_{0}, \mu_{0}\right)$, it follows that a given point $\left(\mu^{\prime}, x_{1}^{\prime}, x_{2}^{\prime}\right)$ belongs to $\widetilde{U}$ provided $x_{1}^{\prime}$ and $x_{2}^{\prime}$ are sufficiently small. If such a point is recurrent, then $\left(\mu^{\prime}, x_{1}^{\prime}, x_{2}^{\prime}\right) \in \widetilde{W}^{c}$. Furthermore, since $\dot{\mu}=0$, the point $\left(\mu^{\prime}, x_{1}^{\prime}, x_{2}^{\prime}\right)$ is recurrent for Eqs. (7.27') if and only if $\left(x_{1}^{\prime}, x_{2}^{\prime}\right)$ is recurrent for Eqs. (7.27). Hence, if $\mu^{\prime} \in\left(-\mu_{0}, \mu_{0}\right)$, all local recurrent points for Eqs. (7.27) belong to $\widetilde{W}^{c}$.

In addition, since $\dot{\mu}=0$, the center manifold $\widetilde{W}^{c}$ is foliated by invariant submanifolds $\widetilde{W}_{\mu}^{c}$, obtained by taking a slice of $\widetilde{W}^{c}$ at a fixed value of $\mu$. When $\mu=0$, the submanifold $\widetilde{W}_{\mu=0}^{c}$ coincides with the original center manifold $W^{c}$ of Eqs. (7.27), and each of these slices is of the same dimension, $\operatorname{dim} \widetilde{W}_{\mu}^{c}=\operatorname{dim} W^{c}$. The geometry of the suspended system is most easily illustrated when the equilibrium at $\left(x_{1}, x_{2}\right)=(0,0)$ happens to persist as $\mu$ varies near $\mu=0$ (as in Hopf bifurcation). In this case we can modify the definition of $x_{1}$ in Eqs. (7.27) so that $x_{1} \in E_{\lambda}$ where $E_{\lambda}$ is the eigenspace associated with the critical eigenvalue $\lambda$, i.e., $E_{\lambda}=E^{c}$ when $\mu=0$. Now Eq. (7.29b) becomes $\widetilde{h}(\mu, 0)=0$, and the manifold $\widetilde{W}^{c}$ is tangent to the subspace defined by $E_{\lambda}$ and the $\mu$ axis as shown in Fig. 18(c). 
Finally, the dynamics on $\widetilde{W}_{\mu}^{c}$ is given by

$$
\frac{d x_{1}}{d t}=V_{1}\left(\mu, x_{1}, \widetilde{h}\left(\mu, x_{1}\right)\right), \mu \in\left(-\mu_{0}, \mu_{0}\right) .
$$

Thus on a neighborhood of criticality center-manifold reduction gives us the autonomous low-dimensional dynamical system (7.30); to rewrite (7.30) in normal form requires the methods of the next section.

\section{POINCARÉ-BIRKHOFF NORMAL FORMS}

At the conclusion of Sec. II, we remarked that the linear spectrum determines the normal form. More precisely, we shall show that the type of spectrum at criticality determines which nonlinear terms are essential and remain after inessential nonlinearities have been removed by a smooth near-identity change of coordinates. We assume that the problem has already been reduced to the appropriate center manifold, and accordingly the specific dynamical systems we consider are one or two dimensional.

For the bifurcations analyzed in Sec. V, normal-form theory is most interesting for Hopf bifurcation and period-doubling bifurcation. For steady-state bifurcation, the lowest-order nonlinear terms are in fact essential, and no particular simplification results from performing normal-form transformations of the type considered here. For this reason, after developing the normal form procedure we work out the application to Hopf bifurcation and period-doubling as examples. Finally we describe some recent theoretical work that explains why normal forms often have greater symmetry than the original dynamical system.

\section{A. Flows}

\section{Generalities}

Center-manifold reduction yields a flow

$$
\begin{aligned}
\frac{d x^{\prime}}{d t}=V\left(\mu, x^{\prime}\right)= & V^{(1)}\left(\mu, x^{\prime}\right)+V^{(2)}\left(\mu, x^{\prime}\right) \\
& +\cdots+V^{(k)}\left(\mu, x^{\prime}\right)+\cdots, \quad x^{\prime} \in \mathbb{R}^{n^{c}},
\end{aligned}
$$

where $n_{c}=\operatorname{dim} E^{c}$ and $V^{(k)}\left(\mu, x^{\prime}\right)$ represents all terms in the Taylor expansion of $V\left(\mu, x^{\prime}\right)$ of order $k$ in $x^{\prime}$. For example, at a Hopf bifurcation $n_{c}=2$ and

$$
V^{(1)}\left(\mu, x^{\prime}\right)=\left[\begin{array}{cc}
\gamma(\mu) & \omega(\mu) \\
-\omega(\mu) & \gamma(\mu)
\end{array}\right]\left[\begin{array}{l}
x_{1}^{\prime} \\
x_{2}^{\prime}
\end{array}\right]
$$

is the appropriate first-order term. For simplicity we have assumed there is no constant term on the right in Eq. (8.1); this need not be true for steady-state bifurcation, but as already mentioned the application to steadystate bifurcation is not of great interest.

Given Eq. (8.1) our goal is to simplify $V\left(\mu, x^{\prime}\right)$ by performing near-identity nonlinear coordinate changes $x^{\prime} \rightarrow x$ that remove as many nonlınear terms as possible. This task is accomplished in an iterative fashion. First we remove $V^{(2)}\left(\mu, x^{\prime}\right)$, then $V^{(3)}\left(\mu, x^{\prime}\right)$, and so forth. The entire procedure can be understood by attempting to construct, if possible, the coordinate change to remove $V^{(k)}\left(\mu, x^{\prime}\right), k \geq 2$. Consider then the coordinate change

$$
x=\Phi\left(x^{\prime}\right)=x^{\prime}+\phi^{(k)}\left(x^{\prime}\right)
$$

with inverse

$$
x^{\prime}=\Phi^{-1}(x)=x-\phi^{(k)}(x)+\mathcal{O}\left(x^{2 k-1}\right),
$$

where

$$
\phi^{(k)}: \mathbb{R}^{n_{c}} \rightarrow \mathbb{R}^{n_{c}}
$$

is a homogeneous polynomial map of degree $k$; i.e., for $a \in \mathbb{R}$,

$$
\phi^{(k)}(a x)=a^{k} \phi^{(k)}(x) .
$$

Aside from Eq. (8.4), we regard $\phi^{(k)}$ as unknown and try to determine the choice of $\phi^{(k)}$ that removes $V^{(k)}$ in (8.1). From Eqs. (8.1) and (8.3a) one has (suppressing the dependence on $\mu$ )

$$
\frac{d x}{d t}=D \Phi\left(x^{\prime}\right) \cdot \frac{d x^{\prime}}{d t}=D \Phi\left(\Phi^{-1}(x)\right) \cdot V\left(\Phi^{-1}(x)\right) .
$$

Now using the expansions

$$
\begin{aligned}
V\left(\Phi^{-1}(x)\right)= & V\left(x-\phi^{(k)}(x)+\mathcal{O}\left(x^{2 k-1}\right)\right) \\
= & V(x)-D V^{(1)}(x) \cdot \phi^{(k)}(x)+\mathcal{O}\left(x^{k+1}\right), \\
D \Phi\left(\Phi^{-1}(x)\right) & =I+D \phi^{(k)}\left(\Phi^{-1}(x)\right) \\
& =I+D \phi^{(k)}(x)+\mathcal{O}\left(x^{2 k-2}\right),
\end{aligned}
$$

we rewrite Eq. (8.5) keeping terms involving $\phi^{(k)}$ up to $\mathcal{O}\left(x^{k}\right)$

$$
\frac{d x}{d t}=V(x)-L\left(\phi^{(k)}\right)+\mathcal{O}\left(x^{k+1}\right),
$$

where

$L\left(\phi^{(k)}\right)(x) \equiv D V^{(1)}(x) \cdot \phi^{(k)}(x)-D \phi^{(k)}(x) \cdot V^{(1)}(x)$.

Our notation is chosen to emphasize that the new terms of $\mathcal{O}\left(x^{k}\right)$ in Eq. (8.7a) are linear in $\phi^{(k)}$ and have the form of a linear operator $L$ acting on $\phi^{(k)}$. Note that $L$, defined by Eq. (8.7b), depends only on the linear term $V^{(1)}(x)$ of the original flow. ${ }^{21}$

\footnotetext{
${ }^{21}$ In connection with Eqs. (8.7b) and (8.8) there are a variety of characterizations in the literature. The linear operator is simply related to the usual Lie bracket of the two vector fields $\phi^{(k)}$ and $V^{(1)}$, i.e., $L\left(\phi^{(k)}\right)=-\left[V^{(1)}, \phi^{(k)}\right]$. Arnold (1988a) refers to Eq. (8.8) as the homological equation associated with the linear operator $D V^{(1)}(x)$. Guckenheimer and Holmes (1986) write the Lie bracket as $\operatorname{ad} V^{(1)}\left(\phi^{(k)}\right)$, since this vector field is induced when $V^{(1)}$ acts on vector fields through the adjoint representation (cf. Olver, 1986).
} 
To remove all terms of $\mathcal{O}\left(x^{k}\right)$ in Eq. (8.7a) we must solve

$$
\boldsymbol{V}^{(k)}(\boldsymbol{x})-\boldsymbol{L}\left(\phi^{(k)}\right)=0
$$

for $\phi^{(k)}(x)$. Formally, this is easy,

$$
\phi^{(k)}(x)=L^{-1}\left(V^{(k)}(x)\right),
$$

but our solution is only sensible if $L^{-1}$ is well defined. The task of finding $L^{-1}$, if it exists, is a problem in finite-dimensional linear algebra. That is, $L$ in Eq. (8.7b) may be viewed as a finite-dimensional matrix, and $L^{-1}$ is well defined if and only if $\operatorname{det} L \neq 0$.
To make this interpretation precise, we go back to Eq. (8.4) and define $\mathscr{H}^{(k)}\left(\mathbb{R}^{n}\right)$,

$\mathscr{H}^{(k)}\left(\mathbb{R}^{n}\right)=\left\{\phi: \mathbb{R}^{n} \rightarrow \mathbb{R}^{n} \mid \phi(a x)=a^{k} \phi(x)\right.$ for all $\left.a \in \mathbb{R}\right\}$,

the space of all homogeneous polynomial maps on $\mathbb{R}^{n}$ of degree $k$. For fixed $k$ and $n, \mathscr{H}^{(k)}\left(\mathbb{R}^{n}\right)$ is a finitedimensional linear vector space. The vector-space structure is obvious, and an example serves to make the finite dimensionality clear. Consider $\mathscr{H}^{(2)}\left(\mathbb{R}^{2}\right)$ with coordinates $(x, y) \in \mathbb{R}^{2} ;$ then any $\phi(x, y) \in \mathscr{H}^{(2)}\left(\mathbb{R}^{2}\right)$ may be written

$$
\begin{aligned}
\phi(x, y) & =\left(\begin{array}{l}
a x^{2}+b x y+c y^{2} \\
d x^{2}+e x y+f y^{2}
\end{array}\right] \\
& =a\left[\begin{array}{c}
x^{2} \\
0
\end{array}\right]+b\left(\begin{array}{c}
x y \\
0
\end{array}\right]+c\left(\begin{array}{l}
y^{2} \\
0
\end{array}\right)+d\left(\begin{array}{c}
0 \\
x^{2}
\end{array}\right)+e\left(\begin{array}{c}
0 \\
x y
\end{array}\right)+f\left(\begin{array}{c}
0 \\
y^{2}
\end{array}\right) .
\end{aligned}
$$

Obviously, $\mathscr{H}^{(2)}\left(\mathbb{R}^{2}\right)$ is six dimensional, and one possible choice of basis is given in Eq. (8.11).

In terms of the spaces $\mathscr{H}^{(k)}$, Eq. (8.4) asserts $\phi^{(k)} \in \mathscr{H}^{(k)}\left(\mathbb{R}^{n^{c}}\right)$ and (8.7) implies

$$
L: \mathscr{H}^{(k)}\left(\mathbb{R}^{n^{c}}\right) \rightarrow \mathscr{H}^{(k)}\left(\mathbb{R}^{n^{c}}\right) ;
$$

thus $L$ is a linear transformation acting on a finitedimensional vector space. Once a basis for $\mathscr{H}^{(k)}\left(\mathbb{R}^{n}{ }^{n}\right)$ is chosen, then $L$ can be written down in matrix form.

Any convenient basis may be selected, since det $L$ is independent of this choice. Recall that $\operatorname{det} L \neq 0$ means that $\phi^{(k)}$ in Eq. (8.9) is well defined and the resulting change of variables (8.3a) will remove all terms of order $k$ in Eq. (8.7a). More generally, however, one finds that $\operatorname{det} L$ depends on $k$ and on whether or not the system is critical; at criticality there will be values of $k$ such that $\operatorname{det} L=0$ because $L$ has at least one zero eigenvalue.

Since $L$ is given in terms of $D V^{(1)}(0)$, it is reasonable to investigate what the condition $\operatorname{det} L=0$ implies about the eigenvalues of $D V^{(1)}(0)$; this is most easily done if we assume that $D V^{(1)}(0)$ can be diagonalized with eigenvalues $\left(\sigma_{1}, \sigma_{2}, \ldots, \sigma_{n_{c}}\right)$. Once Eq. (8.7a) has been written in coordinates that diagonalize $D V^{(1)}(0)$, then the eigenvectors of $L$ are easily found. Let $\phi^{(k)}(x)$ have only a single nonzero component [cf. Eq. (8.11)], $\left(\phi^{(k)}(x)\right)_{l}=\delta_{l j} \phi_{j}^{(k)}(x)$, which we take to be a $k$-degree monomial: $\phi_{j}^{(k)}(x)=x^{\alpha}$ where $j=1, \ldots, n_{c}$ labels the component and the multiindex ${ }^{22} \alpha$ is arbitrary except $|\alpha|=k$. Then applying $L$ to $\phi^{(k)}(x)$ gives

\footnotetext{
${ }^{22}$ In this notation, $\alpha \equiv\left(\alpha_{1}, \alpha_{2}, \ldots, \alpha_{n}\right)$ denotes an $n$-tuple of non-negative integers and $x^{\alpha} \equiv x_{1}^{\alpha_{1}} x_{2}^{\alpha_{2}} \cdots x_{n}^{\alpha_{n}}$. In addition, we define notation $|\alpha| \equiv \alpha_{1}+\alpha_{2}+\cdots+\alpha_{n}$ and, for future reference, $a !=\alpha_{1} ! \alpha_{2} ! \cdots \alpha_{n} !$
}

$$
\begin{aligned}
L\left(\phi^{(k)}(x)\right) & =\sigma_{j} \phi^{(k)}(x)-\left[\sum_{l=1}^{n_{c}} \alpha_{l} \sigma_{l}\right] \phi^{(k)}(x) \\
& =\left[\sigma_{j}-\sum_{l=1}^{n_{c}} \alpha_{l} \sigma_{l}\right] \phi^{(k)}(x) ;
\end{aligned}
$$

hence vectors $\phi^{(k)}(x)$ of this form are eigenvectors, and the eigenvalues have the form $\left[\sigma_{j}-\Sigma_{l=1}^{n_{c}} \alpha_{l} \sigma_{l}\right]$. If we can satisfy the condition

$$
\sigma_{j}=\sum_{l=1}^{n_{c}} \alpha_{1} \sigma_{l}
$$

for any choice of $j$ and $\alpha$ then $L$ has a zero eigenvalue. One can see by inspection that when critical eigenvalues $(\operatorname{Re} \sigma=0)$ occur there are always choices of $\alpha$ which satisfy the "resonance condition" Eq. (8.13). We analyze the case of imaginary eigenvalues in Sec. VIII.A.3 below.

In the presence of such zero eigenvalues, the range of $L$, denoted $L\left(\mathscr{H}^{(k)}\left(\mathbb{R}^{n c}\right)\right)$, is a proper subspace of $\mathscr{H}^{(k)}\left(\mathbb{R}^{n^{c}}\right)$, and we can specify a complementing subspace $C^{(k)}$ so that

$$
\mathscr{H}^{(k)}\left(\mathbb{R}^{n^{c}}\right)=L\left(\mathscr{H}^{(k)}\left(\mathbb{R}^{n^{c}}\right)\right)+C^{(k)} .
$$

Once $C^{(k)}$ is chosen (and the choice is not unique) then the $k$ th order terms $V^{(k)}$ in Eq. (8.7a) may be split accordingly: $V^{(k)}=V_{r}^{(k)}+V_{c}^{(k)}$ with $V_{r}^{(k)} \in L\left(\mathscr{H}^{(k)}\left(\mathbb{R}^{n^{c}}\right)\right)$ and $V_{c}^{(k)} \in C^{(k)}$. The component in the range can be removed,

$$
\phi^{(k)}=L^{-1}\left(V_{r}^{(k)}\right)
$$

leaving behind the "essential" nonlinear terms at order $k$ namely $V_{c}^{(k)}$. In this way $\phi^{(2)}$ is first specified, then $\phi^{(3)}$, 
and so forth so that one generates a power series ${ }^{23}$ representing the desired normal-form transformation to all orders:

$$
x=\Phi\left(x^{\prime}\right)=x^{\prime}+\phi^{(2)}\left(x^{\prime}\right)+\phi^{(3)}\left(x^{\prime}\right)+\cdots .
$$

The normal form resulting from this procedure has the structure

$$
\frac{d x}{d t}=V^{(1)}(x)+V_{c}^{(2)}(x)+V_{c}^{(3)}(x)+\cdots ;
$$

if at order $k, \operatorname{det} L \neq 0$, then of course $V_{c}^{(k)} \equiv 0$.

There is an important subtlety in this procedure. The series Eq. (8.16), representing the transformation $\Phi\left(x^{\prime}\right)$ required to put the original flow Eq. (8.1) into normal form to all orders, typically diverges. Thus, while we can describe which terms can be removed at any given order, the change of variables required to remove them to all orders does not generally exist. In practical applications one implements the transformation to normal form only up to some finite order, and this finite-order approximation to the original flow Eq. (8.1) is studied. The possible effects of the neglected higher-order terms can then be considered in reaching final conclusions.

\section{Steady-state bifurcation on $\mathbb{R}$}

For steady-state bifurcation with a simple zero eigenvalue, $n_{c}=1$ in Eq. (8.1), and $V\left(\mu, x^{\prime}\right)$ has the form described in Eq. (5.2). If we try to simplify Eq. (5.4) by applying the coordinate change Eq. (8.35) to remove the $x^{2}$ term, then the required change of variables is singular at criticality $(\mu=0)$. For this reason, the method of Poincaré-Birkhoff normal forms is not particularly useful in this case. A similar limitation holds for steady-state bifurcation in maps.

\section{Hopf bifurcation on $\mathbb{R}^{2}$}

Generically for Hopf bifurcation, $n_{c}=2$ in Eq. (8.1), and we take $V^{(1)}\left(\mu, x^{\prime}\right)$ as given by Eq. (8.2). Let $(x, y) \in \mathbb{R}^{2}$ denote the coordinates, then $\phi^{(k)} \in \mathscr{H}^{(k)}\left(\mathbb{R}^{2}\right)$ has the form

$$
\phi^{(k)}(x, y)=\left[\begin{array}{l}
\phi_{x}^{(k)}(x, y) \\
\phi_{y}^{(k)}(x, y)
\end{array}\right],
$$

and in these variables $L\left(\phi^{(k)}\right)$ is expressed as

\footnotetext{
${ }^{23}$ Because of the iterative process used to construct $\Phi$, the full series in Eq. (8.16) is not of the form $x^{\prime}+\sum_{k>1} \phi^{(k)}\left(x^{\prime}\right)$.
}

$$
\begin{aligned}
L\left(\phi^{(k)}\right)= & {\left[\begin{array}{cc}
\gamma & \omega \\
-\omega & \gamma
\end{array}\right] \cdot\left[\begin{array}{l}
\phi_{x}^{(k)}(x, y) \\
\phi_{y}^{(k)}(x, y)
\end{array}\right] } \\
& -\left[\begin{array}{cc}
\frac{\partial \phi_{x}^{(k)}}{\partial x} & \frac{\partial \phi_{x}^{(k)}}{\partial y} \\
\frac{\partial \phi_{y}^{(k)}}{\partial x} & \frac{\partial \phi_{y}^{(k)}}{\partial y}
\end{array}\right] \cdot\left[\begin{array}{l}
\gamma x+\omega y \\
\gamma x-\omega y
\end{array}\right] .
\end{aligned}
$$

As noted in Sec. VIII.A.1, to determine the eigenvalues of $L$ it is more convenient to use the complex coordinates $(z, \bar{z})$ that diagonalize $D V^{(1)}(0)$, i.e.,

$$
\left[\begin{array}{l}
z \\
\bar{z}
\end{array}\right]=\left[\begin{array}{cc}
1 & i \\
1 & -i
\end{array}\right]\left[\begin{array}{l}
x \\
y
\end{array}\right] \equiv S \cdot\left(\begin{array}{l}
x \\
y
\end{array}\right),
$$

so that

$$
S^{-1}=\frac{1}{2}\left(\begin{array}{cc}
1 & 1 \\
-i & i
\end{array}\right)
$$

and

$$
S\left[\begin{array}{cc}
\gamma & \omega \\
-\omega & \gamma
\end{array}\right] S^{-1}=\left[\begin{array}{cc}
\gamma-i \omega & 0 \\
0 & \gamma+i \omega
\end{array}\right] .
$$

In terms of $(z, \bar{z})$, the same vector $\phi^{(k)} \in \mathscr{H}^{(k)}\left(\mathbb{R}^{2}\right)$ is reexpressed

$$
\begin{aligned}
\phi^{(k)} & =\left[\begin{array}{l}
\phi_{z}^{(k)}(z, \bar{z}) \\
\phi_{z}^{(k)}(z, \bar{z})
\end{array}\right] \\
& \equiv S \cdot\left[\begin{array}{l}
\phi_{x}^{(k)}(x(z, \bar{z}), y(z, \bar{z})) \\
\phi_{y}^{(k)}(x(z, \bar{z}), y(z, \bar{z}))
\end{array}\right],
\end{aligned}
$$

and the action of $L$ on $\phi^{k}$ is

$$
\begin{aligned}
L\left(\phi^{(k)}\right)= & {\left[\begin{array}{cc}
\gamma-i \omega & 0 \\
0 & \gamma+i \omega
\end{array}\right]\left[\begin{array}{l}
\phi_{z}^{(k)} \\
\phi_{\bar{z}}^{(k)}
\end{array}\right] } \\
& -\left(\begin{array}{cc}
\frac{\partial \phi_{z}^{(k)}}{\partial z} & \frac{\partial \phi_{z}^{(k)}}{\partial \bar{z}} \\
\frac{\partial \phi_{\bar{z}}^{(k)}}{\partial z} & \frac{\partial \phi_{\bar{z}}^{(k)}}{\partial \bar{z}}
\end{array}\right)\left[\begin{array}{l}
(\gamma-i \omega) z \\
(\gamma+i \omega) \bar{z}
\end{array}\right] .
\end{aligned}
$$

By inspection from Eq. (8.20) we see that the eigenvectors have the form $\left(\phi_{z}, 0\right)$ or $\left(0, \phi_{\bar{z}}\right)$, so we introduce the vectors

$$
\left.\begin{array}{l}
\xi_{+}^{(k, l)}(z, \bar{z}) \equiv\left(\begin{array}{c}
z^{l} \bar{z}^{k-l} \\
0
\end{array}\right] \\
\xi_{-}^{(k, l)}(z, \bar{z}) \equiv\left[\begin{array}{c}
0 \\
z^{l} \bar{z}^{k-l}
\end{array}\right)
\end{array}\right\} l=0,1, \ldots, k,
$$

which are eigenvectors of $L$ and also provide a basis for $\mathscr{H}^{(k)}\left(\mathbb{R}^{2}\right)$. From Eq. (8.20) we calculate the eigenvalues

$$
L\left(\xi_{ \pm}^{(k, l)}\right)=\lambda_{ \pm}^{(k, l)}(\mu) \xi_{ \pm}^{(k, l)}
$$


where

$$
\lambda_{ \pm}^{(k, l)}(\mu) \equiv(1-k) \gamma(\mu)-i \omega(\mu)(k-2 l \pm 1) .
$$

Since det $L=0$ implies at least one zero eigenvalue, and a zero eigenvalue in Eq. (8.22b) requires that real and imaginary parts vanish separately, we must satisfy

$$
\begin{aligned}
& (1-k) \gamma(\mu)=0 \\
& (k-2 l \pm 1) \omega(\mu)=0
\end{aligned}
$$

to obtain $\lambda_{ \pm}^{(k, l)}=0$. Because $k \geq 2$, Eq. (8.23a) fails unless $\gamma(\mu)=0$, which requires that we are at criticality $\mu=0$ [recall Eq. (5.23)]. At $\mu=0, \omega(0) \neq 0$, so Eq. (8.23b) requires $k-2 l \pm 1=0$. Since $2 l \pm 1$ is odd, for $k$ even we will never satisfy Eq. (8.22b), and for $k$ odd there are exactly two null eigenvectors at criticality,

$$
\left.\begin{array}{l}
\xi_{+}^{(k,(k+1) / 2)}=\left[\begin{array}{c}
z|z|^{k-1} \\
0
\end{array}\right] \\
\xi_{-}^{(k,(k-1) / 2)}=\left(\begin{array}{c}
0 \\
\bar{z}|z|^{k-1}
\end{array}\right)
\end{array}\right\} k=3,5,7, \ldots .
$$

These two vectors are a natural basis for the complement $C^{(k)}$ to the range of $L$,

$$
C^{(k)}=\operatorname{span}\left\{\xi_{+}^{(k,(k+1) / 2)}, \xi_{-}^{(k,(k-1) / 2)}\right\} k=3,5,7 \ldots .
$$

The implication for Eq. (8.1), written in complex coordinates Eq. (8.19), is a normal form with all even nonlinear terms removed,

$$
\left[\begin{array}{c}
\dot{z} \\
\dot{z}
\end{array}\right]=\left(\begin{array}{cc}
\gamma-i \omega & 0 \\
0 & \gamma+i \omega
\end{array}\right]\left[\begin{array}{l}
z \\
\bar{z}
\end{array}\right]+\sum_{j=1}^{\infty}\left(\begin{array}{c}
\alpha_{j} z|z|^{2 j} \\
\bar{\alpha}_{j} \bar{z}|z|^{2 j}
\end{array}\right) .
$$

Rewriting Eq. (8.25) in polar variables, $z=r e^{-i \theta}$, yields

$$
\begin{aligned}
& \dot{r}=r\left[\gamma(\mu)+\sum_{j=1}^{\infty} a_{j} r^{2 j}\right], \\
& \dot{\theta}=\omega(\mu)+\sum_{j=1}^{\infty} b_{j} r^{2 j},
\end{aligned}
$$

where $a_{j}=\operatorname{Re}\left(\alpha_{j}\right)$ and $b_{j}=-\operatorname{Im}\left(\alpha_{j}\right)$. This is precisely the normal form introduced in Eq. (5.22).

\section{Normal-form symmetry}

Although normal forms may have fewer nonlinear terms, the discussion above does not explain why this should simplify the nonlinear analysis. For example, the one-dimensional logistic map has only one nonlinear term and the Lorenz equations have only two nonlinear terms, yet the immense dynamical complexity of these two systems is well known.

There is a more intrinsic explanation for the practical utility of normal-form theory: normal forms can have greater symmetry than the original system, and this makes them simpler and therefore useful. The phaseshift symmetry of the Hopf normal form, i.e., the covari- ance of Eq. (8.26) under $\theta \rightarrow \theta+\phi$, illustrates this point. Note that this symmetry was not assumed to hold for the original vector field Eq. (8.1); rather, it is introduced by the normal-form transformation Eq. (8.16). As already discussed, the normal-form procedure is formal in the sense that Eq. (8.16) may not converge if carried to all orders. When the series diverges, then a symmetry introduced by Eq. (8.16) describes only an approximate property of Eq. (8.1), even though it is exact for the normal form.

In the case of Hopf bifurcation we constructed the normal form Eq. (8.16) first and then noted the phase-shift symmetry. This order can be reversed; the theory of normal forms can be formulated by identifying the relevant symmetry first and defining the normal form by its symmetry. The advantages of this second approach were noted by Belitskii (1978, 1981), Cushman and Sanders (1986), and Elphick et al.. (1987). The results of Elphick et al (1987) are clearly discussed in Golubitsky, Stewart, and Schaeffer (1988), whose presentation is summarized here.

The key result is that the complementing subspace $C^{(k)}$ in Eq. (8.14) may be defined by a symmetry $\Gamma$ that is determined by the linearization at criticality; i.e., $D V(0,0)$. More precisely, let $M=D V(0,0)$ and $M^{T}=$ (transpose of $M$ ). Then $M^{T}$ generates a one-parameter group of transformations with the obvious multiplication rule

$$
\exp \left(s_{1} M^{T}\right) \exp \left(s_{2} M^{T}\right)=\exp \left[\left(s_{1}+s_{2}\right) M^{T}\right] .
$$

The closure of this one-parameter group defines the normal-form symmetry ${ }^{24}$

$$
\Gamma=\overline{\left\{\exp \left(s M^{T}\right) \mid s \in \mathbb{R}\right\}} \text {. }
$$

Let $\mathcal{H}_{\Gamma}^{(k)}\left(\mathbb{R}^{n}\right)$ denote the subspace of $\mathscr{H}^{(k)}\left(\mathbb{R}^{n}\right)$ comprising those maps with $\Gamma$ symmetry, i.e., those $V^{(k)}(x) \in \mathscr{H}^{(k)}\left(\mathbb{R}^{n}\right)$ such that

$$
V^{(k)}\left(\exp \left(s M^{T}\right) \cdot x\right)=\exp \left(s M^{T}\right) \cdot V^{(k)}(x)
$$

for all $s \in \mathbb{R}$.

We shall prove that $\mathscr{H}_{\Gamma}^{(k)}$ may be taken as the complement $C^{(k)}$ to the range of $L$ so that Eq. (8.14) becomes

$$
\mathscr{H}^{(k)}\left(\mathbb{R}^{n^{c}}\right)=L\left(\mathscr{H}^{(k)}\left(\mathbb{R}^{n^{c}}\right)\right) \oplus \mathcal{H}_{\Gamma}^{(k)}\left(\mathbb{R}^{n^{c}}\right) .
$$

In words, this splitting implies that the normal-form transformation Eq. (8.3a) can remove all $k$ th-order terms except those with $\Gamma$ symmetry.

The argument relies on a clever definition of inner product on $\mathscr{H}^{(k)}\left(\mathbb{R}^{n}\right)$. This definition is based on the following product for monomials: for $x \in \mathbb{R}^{n}$, let $x^{\alpha}$ and $x^{\beta}$ denote two monomials in multi-index notation and define

$$
\left[x^{\alpha}, x^{\beta}\right] \equiv \delta_{\alpha \beta} \alpha !
$$

\footnotetext{
${ }^{24} \mathrm{By}$ defining $\Gamma$ as a closed group of matrices we ensure that it is a Lie group.
} 
which can be conveniently rewritten as

$$
\left[x^{\alpha}, x^{\beta}\right]=\left.\left\{\frac{\partial^{|\alpha|} x^{\beta}}{\partial x^{\alpha}}\right\}\right|_{x=0} .
$$

This bracket extends to polynomials in the obvious way: let $p(x)=\sum_{\alpha} p_{\alpha} x^{\alpha}$ and $q(x)=\sum_{\beta} q_{\beta} x^{\beta}$. Then

$$
\begin{aligned}
{[p(x), q(x)] } & =\sum_{\alpha, \beta} p_{a} q_{\beta}\left[x^{\alpha}, x^{\beta}\right] \\
& =\left.\sum_{\alpha} p_{\alpha}\left\{\frac{\partial^{|\alpha|} q(x)}{\partial x^{\alpha}}\right\}\right|_{x=0} .
\end{aligned}
$$

Finally, given $\phi, \psi \in \mathscr{H}^{(k)}\left(\mathbb{R}^{n}\right)$, we define their inner product by

$$
\langle\phi, \psi\rangle \equiv \sum_{j=1}^{n}\left[\phi_{j}(x), \psi_{j}(x)\right],
$$

where $\phi_{j}$ and $\psi_{j}$ are the jth components of $\phi$ and $\psi$, respectively.

At criticality, the operator $L$ in Eq. (8.7b) becomes

$$
L_{M}(\phi)(x)=M \cdot \phi(x)-D \phi(x) \cdot M \cdot x
$$

where $L_{M}$ has been written with a subscript to emphasize the dependence on $M$. Given the inner product (8.32), we define the adjoint of $L_{M}$ as that operator satisfying

$$
\left\langle L_{M}^{\dagger} \phi, \psi\right\rangle=\left\langle\phi, L_{M} \psi\right\rangle
$$

for all $\phi$ and $\psi$ in $\mathscr{H}^{(k)}\left(\mathbb{R}^{n}\right)$. We shall determine $L_{M}^{\dagger}$ as

$$
\begin{aligned}
L_{M}^{\dagger}(\phi)(x) & =M^{T} \cdot \phi(x)-D \phi(x) \cdot M^{T} \cdot x \\
& =L_{M^{T}}(\phi)(x)
\end{aligned}
$$

by applying two identities,

$$
\begin{aligned}
& \left\langle\phi(x), A^{T} \cdot \psi(x)\right\rangle=\langle A \cdot \phi(x), \psi(x)\rangle, \\
& \left\langle\phi(x), \psi\left(A^{T} \cdot x\right)\right\rangle=\langle\phi(A \cdot x), \psi(x)\rangle,
\end{aligned}
$$

that hold for any linear transformation $A: \mathbb{R}^{n} \rightarrow \mathbb{R}^{n}$. The first identity follows immediately from Eqs. (8.31) and (8.32):

$$
\begin{aligned}
\left\langle\phi(x), A^{T} \cdot \psi(x)\right\rangle & =\sum_{i, j=1}^{n}\left[\phi_{j}(x),\left(A^{T}\right)_{j i} \phi_{i}(x)\right] \\
& =\sum_{i, j=1}^{n}\left[A_{i j} \phi_{j}(x), \phi_{i}(x)\right] \\
& =\langle A \cdot \phi(x), \psi(x)\rangle .
\end{aligned}
$$

For the second identity, we express the $j$ th component of $\phi$ as $\phi_{j}(x)=\sum_{\alpha} \phi_{j, \alpha} x^{\alpha}$ [and similarly for $\psi_{j}(x)$ ], so that the left-hand side of Eq. (8.37) becomes

$$
\left\langle\phi(x), \psi\left(A^{T} \cdot x\right)\right\rangle=\sum_{j=1}^{n} \sum_{\alpha, \beta} \phi_{j, \alpha} \psi_{j, \beta}\left[x^{\alpha},\left(A^{T} \cdot x\right)^{\beta}\right] .
$$

Then with Eq. (8.31) we have

$$
\begin{aligned}
{\left[x^{\alpha},\left(A^{T} \cdot x\right)^{\beta}\right] } & =\left.\left\{\frac{\partial^{|\alpha|}\left(A^{T} \cdot x\right)^{\beta}}{\partial x^{\alpha}}\right\}\right|_{x=0} \\
& =\left.\left\{\left[A \cdot \frac{\partial}{\partial x}\right]^{\alpha} x^{\beta}\right\}\right|_{x=0} \\
& =\left[(A \cdot x)^{\alpha}, x^{\beta}\right] ;
\end{aligned}
$$

in the second step the change of variables $y=A^{T} \cdot x$ and the chain rule,

$$
\frac{\partial y^{\beta}}{\partial x}=A \cdot \frac{\partial}{\partial y}\left(y^{\beta}\right)
$$

were used to justify the substitution ${ }^{25}$

$$
\left\{\frac{\partial^{|\alpha|}\left(A^{T} \cdot x\right)^{\beta}}{\partial x^{\alpha}}\right\}=\left\{\left[A \cdot \frac{\partial}{\partial y}\right]^{\alpha} y^{\beta}\right\} .
$$

With Eqs. (8.39) and (8.40), the second identity (8.37) follows directly.

These identities are applied by choosing $A^{T}=\exp (-s M)$ in Eq. (8.36) and $A^{T}=\exp (s M)$ in Eq. (8.37) to obtain

$$
\left\langle\phi(x), e^{-s M} \psi\left(e^{s M} \cdot x\right)\right\rangle=\left\langle e^{-s M^{T}} \phi\left(e^{s M^{T}} \cdot x\right), \psi(x)\right\rangle .
$$

By differentiating Eq. (8.41) with respect to $s$ at $s=0$, we finally arrive at

$$
\left\langle\phi(x), L_{M}(\psi)(x)\right\rangle=\left\langle L_{M} T(\phi)(x), \psi(x)\right\rangle,
$$

which establishes Eq. (8.35).

The argument leading to Eq. (8.29) can now be summarized. The vector space $\mathscr{H}^{(k)}\left(\mathbb{R}^{n}\right)$ is first written as the direct sum of the kernel of $L_{M}^{\dagger}$ and the orthogonal complement of the kernel:

$$
\mathscr{H}^{(k)}\left(\mathbb{R}^{n}\right)=\left(\operatorname{ker} L_{M}^{\dagger}\right)^{\perp} \oplus\left(\operatorname{ker} L_{M}^{\dagger}\right) ;
$$

then the Fredholm alternative ${ }^{26}$ for $L_{M}$ implies $\left(\operatorname{ker} L_{M}^{\dagger}\right)^{\perp}=L\left(\mathscr{H}^{(k)}\left(\mathbb{R}^{n}\right)\right)$ and Eq. (8.35) implies $\operatorname{ker} L_{M}^{\dagger}=\operatorname{ker} L_{M^{T}}$. Thus Eq. (8.43) may be reexpressed as

$$
\mathscr{H}^{(k)}\left(\mathbb{R}^{n}\right)=L\left(\mathcal{H}^{(k)}\left(\mathbb{R}^{n}\right)\right) \oplus\left(\operatorname{ker} L_{M^{T}}\right) .
$$

Finally, with the aid of the identity

$$
\frac{d}{d s}\left(e^{s M^{T}} \cdot \phi\left(e^{-s M^{T}} \cdot x\right)\right)=e^{s M^{T}} L_{M^{T}}(\phi)\left(e^{-s M^{T}} \cdot x\right),
$$

we can identify $\operatorname{ker} L_{M^{T}}$ with $\mathscr{H}_{\Gamma}^{(k)}\left(\mathbb{R}^{n}\right)$. If $\phi \in \mathcal{H}_{\Gamma}^{(k)}\left(\mathbb{R}^{n}\right)$,

$$
\left[A \cdot \frac{\partial}{\partial y}\right]^{\alpha} \equiv\left(A_{1 l_{1}} \frac{\partial}{\partial y_{l_{1}}}\right)^{\alpha_{1}}\left(A_{2 l_{2}} \frac{\partial}{\partial y_{l_{2}}}\right)^{\alpha_{2}} \ldots\left(A_{n l_{n}} \frac{\partial}{\partial y_{l_{n}}}\right)^{\alpha_{n}}
$$

(summation on repeated indices).

${ }^{26}$ See Stakgold (1979), pp. 321-323. 
then the left-hand side of Eq. (8.45) vanishes, which implies $L_{M^{T}} \phi=0 ;$ hence $\phi \in \operatorname{ker} L_{M^{T}}$. Conversely, if $\phi \in \operatorname{ker} L_{M^{T}}$, then the right-hand side is zero and the lefthand side must be independent of $s$. This implies

$$
e^{s M^{T}} \cdot \phi\left(e^{-s M^{T}} \cdot x\right)=\phi(x),
$$

since $\phi(x)$ is the value at $s=0$; hence $\phi \in \mathcal{H}_{\Gamma}^{(k)}\left(\mathbb{R}^{n}\right)$. Thus $\operatorname{ker} L_{M^{T}}=\mathscr{H}_{\Gamma}^{(k)}\left(\mathbb{R}^{n}\right)$, and Eq. (8.29) is established.

Note that when $M$ can be diagonalized then we may assume $M^{T}=-M$ and consequently $\operatorname{ker} L_{M^{T}}=\operatorname{ker} L_{M}$. In this case, the definition of $\Gamma$ can be based directly on $M$; it is not necessary to use the transpose.

In our example of Hopf bifurcation, the linearization at criticality gives

$$
M^{T}=\left[\begin{array}{cc}
0 & \omega(0) \\
-\omega(0) & 0
\end{array}\right]
$$

so that an element of $\Gamma$ has the form

$$
\exp \left(s M^{T}\right)=\left[\begin{array}{cc}
\cos (s \omega(0)) & -\sin (s \omega(0)) \\
\sin (s \omega(0)) & \cos (s \omega(0))
\end{array}\right]
$$

As expected, this identifies the normal-form symmetry for Hopf bifurcation as rotations in $\theta$ or $\Gamma=S^{1}$. Note that for steady-state bifurcations $D V(0,0)=0$, so the associated $\Gamma$ in Eq. (8.27) is trivial, consisting only of the identity matrix. This explains why Poincaré-Birkhoff normal-form methods do not significantly simplify the analysis of a steady-state bifurcation.

\section{B. Maps}

\section{Generalities}

On the center manifold we find a map that may be written

$$
\begin{array}{r}
x_{j+1}^{\prime}=f\left(x_{j}^{\prime}\right)=f^{(1)}\left(x_{j}^{\prime}\right)+f^{(2)}\left(x_{j}^{\prime}\right)+\cdots \\
x^{\prime} \in \mathbb{R}^{n^{n}}
\end{array}
$$

in a notation modeled on Eq. (8.1). We suppress explicit parameter dependence and ignore constant terms as before. The goal remains the same: remove $f^{(k)}\left(x^{\prime}\right)$, if possible, using the change of coordinates Eq. (8.3). In the new variables (unprimed) we find

$$
\begin{aligned}
x_{j+1} & =f\left(\Phi^{-1}\left(x_{j}\right)\right)+\phi^{(k)}\left(f\left(\Phi^{-1}\left(x_{j}\right)\right)\right) \\
& =f\left(x_{j}\right)-L\left(\phi^{(k)}\right)\left(x_{j}\right)+\mathcal{O}\left(x^{k+1}\right),
\end{aligned}
$$

where now $L$ is defined by

$L\left(\phi^{(k)}\right)(x)=D f^{(1)}(0) \cdot \phi^{(k)}(x)-\phi^{(k)}\left(f^{(1)}(x)\right)$.

Note that Eq. (8.50b) differs crucially from (8.7b) in the second term; nevertheless we are again seeking to solve an equation of the same form,

$$
f^{(k)}(x)-L\left(\phi^{(k)}\right)(x)=0,
$$

by constructing $L^{-1}$. When $\operatorname{det} L=0$, there are zero eigenvalues, and some nonlinear terms cannot be eliminated. As for vector-field normal forms, if we assume coordinates can be found which diagonalize $D f^{(1)}(0)$, then the vectors $\phi^{(k)}(x)$ having a single monomial component $\phi_{j}^{(k)}(x)=x^{\alpha}$ will be eigenvectors for $L$. Let $\left(\sigma_{1}, \sigma_{2}, \ldots, \sigma_{n_{c}}\right)$ denote the eigenvalues of $D f^{(1)}(0)$. Then we find

$$
\begin{aligned}
L\left(\phi^{(k)}\right)(x) & =\sigma_{j} \phi^{(k)}(x)-\sigma^{\alpha} \phi^{(k)}(x) \\
& =\left[\sigma_{j}-\sigma^{\alpha}\right] \phi^{(k)}(x)
\end{aligned}
$$

from Eq. (8.50b), where $\sigma^{\alpha} \equiv \sigma_{1}^{\alpha_{1}} \sigma_{2}^{\alpha_{2}} \cdots \sigma_{n_{c}{ }^{\alpha} \text {; }}^{\alpha_{1}}$, hence for maps the resonance condition required for a zero eigenvalue is

$$
\sigma_{j}=\sigma^{\alpha}
$$

for some choice of $j$ and $\alpha$.

When zero eigenvalues occur, then the nonlinear terms that cannot be removed may be characterized by their symmetry. Let $M=D f^{(1)}(0)$ denote the linear map at criticality [cf. Eq. (2.10)] and define the group generated by $M^{T}$,

$$
\Gamma=\overline{\left\{\left(M^{T}\right)^{n} \mid n=\text { integer }\right\}},
$$

so that $\mathscr{H}_{\Gamma}^{(k)}\left(\mathbb{R}^{n}\right)$ now denotes elements of $\mathscr{H}^{(k)}\left(\mathbb{R}^{n}\right)$ with symmetry (8.54); i.e., $\phi(x) \in \mathscr{H}_{\Gamma}^{(k)}\left(\mathbb{R}^{n}\right) \quad$ requires $M^{T} \cdot \phi(x)=\phi\left(M^{T} \cdot x\right)$. With $\Gamma$ and $\mathscr{H}_{\Gamma}^{(k)}$ redefined in this way the proof that $\mathscr{H}^{(k)}\left(\mathbb{R}^{n^{c}}\right)$ may be expressed as

$$
\mathscr{H}^{(k)}\left(\mathbb{R}^{n^{c}}\right)=L\left(\mathcal{H}^{(k)}\left(\mathbb{R}^{n^{c}}\right)\right) \oplus \mathcal{H}_{\Gamma}^{(k)}\left(\mathbb{R}^{n^{c}}\right)
$$

is quite similar to the argument leading to Eq. (8.29). With

$$
L_{M}\left(\phi^{(k)}\right)(x)=M \cdot \phi^{(k)}(x)-\phi^{(k)}(M \cdot x)
$$

denoting the operator $L$ [cf. Eq. (8.50b)] at criticality, the identities (8.36) and (8.37) imply $L_{M}^{\dagger}=L_{M}$. Therefore $\left(\operatorname{ker} L_{M}^{\dagger}\right)^{\perp}=L_{M}\left(\mathcal{H}^{(k)}\right)$ and $\operatorname{ker} L_{M}^{\dagger}=\operatorname{ker} L_{M^{T}}$ hold as before, and we obtain

$$
\mathscr{H}^{(k)}\left(\mathbb{R}^{n^{c}}\right)=L_{M}\left(\mathscr{H}^{(k)}\left(\mathbb{R}^{n^{c}}\right)\right) \oplus \operatorname{ker} L_{M^{T}}
$$

by the same reasoning that led to Eq. (8.44). It is only necessary to check that $\operatorname{ker} L_{M}=\mathscr{H}_{\Gamma}^{(k)}\left(\mathbb{R}^{{ }^{n}}\right)$ still holds. This follows by noting that $\phi \in \operatorname{ker} L_{M^{T}}$ if and only if

$$
M^{T} \cdot \phi(x)=\phi\left(M^{T} \cdot x\right),
$$

which in turn is also necessary and sufficient for $\phi \in \mathscr{H}_{\Gamma}^{(k)}\left(\mathbb{R}^{n_{c}}\right)$.

The splitting (8.55) has the same significance here as in the vector-field case: only when $\Gamma$ defines a nontrivial symmetry should we expect the Poincaré-Birkhoff normal form to be simpler than the original map. In addition, the normal form for the original map (8.49) will be 


$$
f(x)=f^{(1)}(x)+f_{c}^{(2)}(x)+f_{c}^{(3)}(x)+\cdots
$$

where $f_{c}^{(k)}(x) \in \mathscr{H}_{\Gamma}^{(k)}\left(\mathbb{R}^{n^{c}}\right)$.

\section{Period-doubling bifurcation on $\mathbb{R}^{1}$}

Typically $n_{c}=1$ for a period-doubling bifurcation, and Eq. (8.49) is a map in one dimension with

$$
f^{(1)}(\mu, x)=\lambda(\mu) x
$$

where

$$
\lambda(0)=-1, \frac{d \lambda}{d \mu}(0)<0 .
$$

The space $\mathscr{H}^{(k)}(\mathbb{R})$ is one dimensional for all $k$, and the single basis vector,

$$
\xi^{(k)}(x) \equiv x^{k}
$$

is ' an eigenvector for $L: \mathscr{H}^{(k)}(\mathbb{R}) \rightarrow H^{(k)}(\mathbb{R})$; from Eq. $(8.50 \mathrm{~b})$ we find

$$
L\left(\xi^{(k)}(x)\right)=\lambda(\mu)\left(1-\lambda(\mu)^{k-1}\right) \xi^{(k)}(x) .
$$

Since $\lambda(0)=-1$, eigenvalue $\lambda\left(1-\lambda^{k-1}\right)$ will vanish at criticality when $k$ is odd; thus terms of even degree can be removed, and only odd terms,

$$
-\xi(x)=\xi(-x),
$$

will remain in the normal form. If we consider the expected symmetry $\Gamma$ in Eq. (8.54), then $M=D_{x} f^{(1)}(0,0)=-I$ so $\Gamma=Z_{2}(-I)$, the two-element group on $\mathbb{R}$ generated by $-I$. Thus we are again led to the conclusion that for period-doubling the normal form,

$$
x_{j+1}=\lambda(\mu) x_{j}\left[1+\alpha_{1} x_{j}^{2}+\alpha_{2} x_{j}^{4}+\mathcal{O}\left(x^{6}\right)\right] \text {, }
$$

for Eq. (8.49) will have a reflection symmetry as claimed in Eqs. (5.34) and (5.35).

\section{Hopf bifurcation on $\mathbb{R}^{2}$}

As for flows, one expects $n_{c}=2$ for Hopf bifurcation, and with coordinates $(x, y)$ on $\mathbb{R}^{2}$ we have for $0<\theta<\frac{1}{2}$

$$
f^{(1)}(x, y)=\left(\begin{array}{cc}
{[1+a(\mu)] \cos 2 \pi \theta(1+b(\mu))} & -[1+a(\mu)] \sin 2 \pi \theta(1+b(\mu)) \\
{[1+a(\mu)] \sin 2 \pi \theta(1+b(\mu))} & {[1+a(\mu)] \cos 2 \pi \theta(1+b(\mu))}
\end{array}\right)\left(\begin{array}{l}
x \\
y
\end{array}\right)
$$

in Eq. (8.32), where $a(\mu), b(\mu)$ satisfy the assumptions in Eq. (5.39). At criticality, $a(0)=b(0)=0$, so the expected symmetry (8.54) will be generated by

$$
M=\left(\begin{array}{cc}
\cos 2 \pi \theta & -\sin 2 \pi \theta \\
\sin 2 \pi \theta & \cos 2 \pi \theta
\end{array}\right)
$$

the rotation matrix for the angle $\theta$ determined by the critical eigenvalues.

As before it is convenient to introduce complex coordinates $(8.19)$ so that $(8.63)$ becomes

$$
S D f^{(1)} S^{-1}=\left(\begin{array}{cc}
\lambda(\mu) & 0 \\
0 & \lambda(\mu)
\end{array}\right),
$$

where $\lambda(\mu) \equiv[1+a(\mu)] e^{i 2 \pi \theta[1+b(\mu)]}$. From Eq. (8.50b) we obtain

$$
L\left(\phi^{(k)}\right)=\left[\begin{array}{ll}
\lambda & 0 \\
0 & \bar{\lambda}
\end{array}\right]\left[\begin{array}{l}
\phi_{z}^{(k)}(z, \bar{z}) \\
\phi_{\bar{z}}^{(k)}(z, \bar{z})
\end{array}\right]-\left[\begin{array}{l}
\phi_{z}^{(k)}(\lambda z, \bar{\lambda} \bar{z}) \\
\phi_{\bar{z}}^{(k)}(\lambda z, \bar{\lambda} \bar{z})
\end{array}\right]
$$

The eigenvectors of $L$ are again given by Eq. (8.21), and (8.65) yields

$$
L\left(\xi_{ \pm}^{(k, l)}\right)=\lambda_{ \pm}^{(k, l)}(\mu) \xi_{ \pm}^{(k, l)}
$$

where

$$
\lambda_{ \pm}^{(k, l)}(\mu)=[1+a(\mu)] e^{ \pm i 2 \pi \theta[1+b(\mu)]}\left[1-(1+a)^{k-1} e^{-i 2 \pi \theta(1+b)(k-2 l \pm 1)}\right] .
$$

By inspection $\operatorname{det} L \neq 0$ unless $\mu=0$, in which case $\lambda_{ \pm}^{(k, l)}(0)=0$ if and only if

$$
\exp [-i 2 \pi \theta(k-2 l \pm 1)]=1 \text {. }
$$

The solutions $(k, l)$ to Eq. (8.68) vary depending on whether $\theta$ is irrational or rational. (a) $\theta$ irrational.

To satisfy Eq. (8.68) requires

$$
\theta(k-2 l \pm 1)=m,
$$

with $m$ an integer, and when $\theta$ is irrational we must have $k-2 l \pm 1=0$. This leads back to the null eigenvectors 
(8.24) found for the Hopf normal form for flows. The resulting normal form in this case is

$$
z_{j+1}=z_{j}\left[\lambda(\mu)+\sum_{i=1}^{\infty} \alpha_{i}\left|z_{j}\right|^{2 i}\right] .
$$

In polar variables, $z=r e^{i \psi}$, we have

$$
\begin{aligned}
& r_{j+1}=[1+a(\mu)] r_{j}\left[1+\sum_{i=1}^{\infty} a_{i} r_{j}^{2 i}\right], \\
& \psi_{j+1}=\psi_{j}+2 \pi \theta[1+b(\mu)]+\sum_{i=1}^{\infty} b_{i} r_{j}^{2 i},
\end{aligned}
$$

where $a_{i}=\operatorname{Re} \alpha_{i}$ and $b_{i}=\operatorname{Im} \alpha_{i}$. This agrees with Eq. (5.41) in Sec. V.

The fact that the dynamics of the amplitude (8.71a) decouples from the phase $(8.71 b)$ reflects the symmetry $\Gamma$. For $\theta$ irrational, the matrices,

$$
M^{n}=\left(\begin{array}{cc}
\cos 2 \pi n \theta & -\sin 2 \pi n \theta \\
\sin 2 \pi n \theta & \cos 2 \pi n \theta
\end{array}\right)
$$

for all integers $n$, provide a dense subset of the group of rotations in the phase. Thus $\Gamma=S^{1}$ is precisely this rotation group and corresponds to the phase-shift symmetry of the normal form (8.71).

(b) $\theta$ rational.

Let $\theta=p / q$ with $0<p / q<\frac{1}{2}$ where the integers $p$ and $q$ are relatively prime. ${ }^{27}$ Now in addition to the solutions $k-2 l \pm 1=0$ for Eq. (8.69) we have another set of solutions represented by

$$
k-2 l \pm 1=n q, \quad n= \pm 1, \pm 2, \ldots
$$

so that $p n=m$. We are primarily concerned with solutions to Eq. (8.73) that introduce new low-order terms into the normal form (8.71). Examination of different cases for (8.73) shows that if $q=3$ or $q=4$ then we get new terms at quadratic and cubic orders, respectively. For $q \geq 5$ the new terms in the normal form are at least fourth order and can be shown to have negligible effect on the analysis of Sec. V. The low-order "resonant" terms are as follows: for $q=3$,

$$
\xi_{+}^{(2,0)}(z, \bar{z})=\left(\begin{array}{c}
\bar{z}^{2} \\
0
\end{array}\right), \quad \xi_{-}^{(2,2)}(z, \bar{z})=\left(\begin{array}{c}
0 \\
z^{2}
\end{array}\right)
$$

are null eigenvectors, and for $q=4$,

$$
\xi_{+}^{(3,0)}(z, \bar{z})=\left(\begin{array}{c}
\bar{z}^{3} \\
0
\end{array}\right), \quad \xi_{-}^{(3,3)}(z, \bar{z})=\left(\begin{array}{c}
0 \\
z^{3}
\end{array}\right)
$$

are the null eigenvectors. Provided $q \neq 3,4$ [or, equivalently, assuming the nonresonance condition (5.40)], the normal form up to third order is given correctly by Eq. (5.42).

\footnotetext{
${ }^{27}$ Two integers are relatively prime if they have no common divisor besides 1 .
}

When $\theta$ is rational, the symmetry $\Gamma$ of the normal form is reduced to $\Gamma=Z_{q}$, the discrete subgroup of $S^{1}$ generated by rotation through $2 \pi / q$. For the cases of "strong resonance," $q=3$ and $q=4$, we are thus led to study maps that are covariant under rotations by $2 \pi / 3$ and $\pi / 2$, respectively, and the structure of the bifurcation is much richer (Arnold, 1988a). [In particular, for $q=4$, there are at least 48 different local phase portraits possible (Arnold, 1989).]

\section{APPLICATIONS}

The normal-form equations provide the most elementary examples of the bifurcations we have considered. However, in practice lengthy calculations may be necessary to extract the relevant normal-form coefficients from the initial equations expressed in physical variables. In this section we analyze bifurcations in two equations that illustrate both the power of center-manifold reduction and the computations required to obtain detailed predictions for specific problems. In addition, each of these applications illustrates new features of the theory that can arise when one encounters equations that have symmetry or that depend on more than one parameter.

The first problem considers a simplified model in plasma physics for the three-wave interaction between an unstable plasma wave and two damped waves. The amplitude equations for the waves lead us to a Hopf bifurcation in a three-dimensional flow that depends on two parameters. The calculations required to obtain the Hopf normal form (5.22) are carried out in detail. Because this model contains two free parameters, the cubic coefficient $a$, evaluated at criticality [cf. Eq. (5.24)] is a function of the remaining parameter. By varying this additional parameter we are able to locate a degenerate bifurcation in which the nondegeneracy condition (5.24) fails, and higher-order terms in the normal form must be considered. This degeneracy allows us to detect and analyze a secondary saddle-node bifurcation for the Hopf limit cycle.

In the second application, we study steady-state bifurcations in the (real) Ginzburg-Landau equation. This analysis illustrates center-manifold reduction for bifurcations in infinite dimensions, i.e., for a partial differential equation. Because the Ginzburg-Landau equation is relatively simple we are able to calculate not only the initial bifurcation from the "trivial" equilibrium but also the secondary bifurcations from the resulting "pure-mode", solutions. These secondary bifurcations are the mechanism for the Eckhaus instability, which plays an important role in the theory of spatially extended patternforming systems (Eckhaus, 1965). The center-manifold reductions in this case are complicated by the fact that the Ginzburg-Landau equation is highly symmetric. In the simplest case-one dimension and periodic boundary conditions - the symmetry group is $O(2) \times O(2)$. Although one typically expects one-dimensional center manifolds at a steady-state bifurcation, in this example 
the initial bifurcation has a four-dimensional center manifold, and the secondary bifurcations lead to twodimensional center manifolds. In each case the symmetry forces the zero eigenvalue to have multiple eigenvectors (four and two, respectively), and this multiplicity leads to larger center manifolds.

\section{A. Hopf bifurcation in a three-wave interaction}

Nonlinear plasma theory involves, in part, an analysis of the interactions between the various waves supported by the plasma. In this example we examine the saturation of a linearly unstable plasma wave via a "three-wave interaction" in a simplified model considered originally by Vyshkind and Rabinovich (1976), Wersinger et al. (1980), and others. Physically, an unstable highfrequency wave is coupled to two damped waves of lower frequency. Under suitable conditions an overall balance results between high-frequency growth and lowfrequency decay. This produces a stable equilibrium in the dynamics of the three waves, and the wave amplitudes are time independent. If, however, the parameters of the interaction are varied to produce less damping or less effective coupling, then this stable balance is destroyed and some form of time-dependent state emerges. For the model considered here, this transition occurs via a Hopf bifurcation. In addition, if the damping of the stable modes is decreased sufficiently, one expects on physical grounds that they may fail to arrest the growth of the unstable mode. In the model, this failure is marked by a shift from supercritical Hopf bifurcation $\left(a_{1}<0\right)$ to subcritical Hopf bifurcation $\left(a_{1}>0\right)$. The calculation of $a_{1}$ allows the location of this transition to be predicted, and the normal-form analysis yields a detailed understanding of the dynamics near this critical region.

For simplicity we assume the two stable waves have equal damping rates $\Gamma$ and equal amplitudes $a_{2}=a_{3}$. The dynamical variables are then $\left(a_{1}, a_{2}, \phi\right)$, where $a_{1}$ is the amplitude of the unstable wave and $\phi=\phi_{1}-\phi_{2}-\phi_{3}$ is the phase difference between the waves. Following Wersinger et al. (1980), we introduce the coordinates $(x, y, z)=\left(a_{1} \cos \phi, a_{1} \sin \phi, a_{2}^{2}\right)$, so that the wave interaction is described by

$$
\frac{d}{d t}\left(\begin{array}{l}
x \\
y \\
z
\end{array}\right)=\left(\begin{array}{ccc}
1 & -\Omega & 1 \\
\Omega & 1 & 0 \\
0 & 0 & -2 \Gamma
\end{array}\right)\left(\begin{array}{l}
x \\
y \\
z
\end{array}\right)+2\left(\begin{array}{c}
-y^{2} \\
x y \\
-x z
\end{array}\right)
$$

where $\Omega=\omega_{1}-\omega_{2}-\omega_{3}$ measures the detuning from the resonance $\omega_{1}=\omega_{2}+\omega_{3}$. Both parameters $\Omega$ and $\Gamma$ are non-negative. For additional background on the plasma physics ancestry of Eq. (9.1) see Wersinger et al. (1980). The chaotic dynamics of the model in the regime of large damping $(\Gamma \rightarrow \infty$ and $\Omega / \Gamma$ fixed) has been analyzed by Hughes and Proctor (1990). The analysis of the Hopf bifurcation in these equations follows Crawford (1983).

Let $V(x, y, z)$ denote the two-parameter family of vector fields defined by the model (9.1), leaving the depen- dence on $(\Omega, \Gamma)$ implicit. The divergence of this family is

$$
\operatorname{div} V=2(1-\Gamma) \text {. }
$$

For $\Gamma<1$ the flow expands volumes in $\mathbb{R}^{3}$ and there are no stable bounded solutions; for $\Gamma>1$ the flow contracts volumes (Verhulst, 1990). Since the equations are unchanged by the shift $(\Omega, y) \rightarrow(-\Omega,-y)$, we may assume $\Omega$ to be non-negative.

\section{Linear analysis}

$V$ has two fixed points. There is a trivial fixed point at $(x, y, z)=(0,0,0)$ corresponding to no waves; this solution is unstable, since the high-frequency wave is unstable. There is a nontrivial fixed point at

$$
\left(x_{0}, y_{0}, z_{0}\right)=\left[-\Gamma, \frac{-\Omega \Gamma}{2 \Gamma-1}, \Gamma\left[1+\frac{\Omega^{2}}{(2 \Gamma-1)^{2}}\right]\right),
$$

whose stability depends on $\Omega$ and $\Gamma$. If we shift the origin to $\left(x_{0}, y_{0}, z_{0}\right), x=x^{\prime}+x_{0}, y=y^{\prime}+y_{0}, z=z^{\prime}+z_{0}$ and drop the primes, then

$\frac{d}{d t}\left(\begin{array}{l}x \\ y \\ z\end{array}\right)=\left(\begin{array}{ccc}1 & (1+\mu) \rho & 1 \\ -\rho & 1-\mu & 0 \\ -\mu\left(1+\rho^{2}\right) & 0 & 0\end{array}\right)\left(\begin{array}{l}x \\ y \\ z\end{array}\right)+2\left(\begin{array}{c}-y^{2} \\ x y \\ -x z\end{array}\right)$,

where $\mu \equiv 2 \Gamma$ and $\rho \equiv \Omega /(\mu-1)$. The eigenvalues $\lambda$ of the linearization at $(x, y, z)=(0,0,0)$ satisfy

$\lambda^{3}+(\mu-2) \lambda^{2}+\left[1+(1+2 \mu) \rho^{2}\right] \lambda+\mu(\mu-1)\left(1+\rho^{2}\right)=0$.

For $\mu \geq 2$, all coefficients are non-negative and the con-

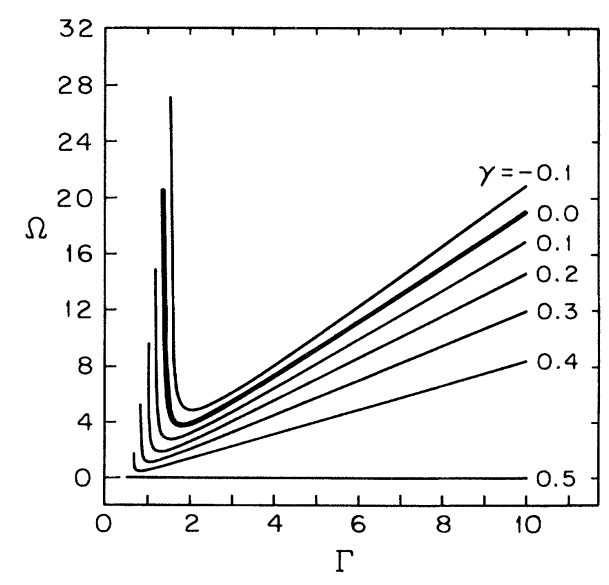

FIG. 19. Surfaces of constant $\gamma$ in the $(\Omega, \Gamma)$ parameter space. The Hopf bifurcation surface is $\gamma=0$; for $\gamma<0$ the fixed point (9.3) is stable. 
stant term is positive. This implies that any real root must be negative; in particular, $\lambda=0$ cannot occur in this region of parameter space. If eigenvalues with $\operatorname{Re} \lambda=0$ occur, they must form a conjugate pair $\pm i \omega$. Thus, in the regions of parameter space where the stability of the fixed point changes, there will be a negative real eigenvalue and a conjugate pair. From the characteristic polynomial, a complex root, $\gamma+i \omega$, satisfies

$$
3 \gamma^{2}-\omega^{2}+2(\mu-2) \gamma+1+\rho^{2}(1+2 \mu)=0 \text {, }
$$

$$
\begin{aligned}
& \gamma\left(\gamma^{2}-3 \omega^{2}\right)+(\mu-2)\left(\gamma^{2}-\omega^{2}\right) \\
& \quad+\left[1+\rho^{2}(1+2 \mu)\right] \gamma+\mu(\mu-1)\left(1+\rho^{2}\right)=0 .
\end{aligned}
$$

Although $(\Omega, \mu)$ are the physical parameters, $\gamma$ and $\mu$ are more convenient, as $\gamma$ directly measures the distance in parameter space from criticality for a Hopf bifurcation, i.e., $\gamma=0$. We may express the dependence of $\Omega$ on $(\gamma, \mu)$ by solving Eq. (9.6) for $\omega^{2}$, eliminating $\omega^{2}$ from Eq. (9.7), and solving for $\rho^{2}$ :

$$
\rho^{2}=\frac{(1-2 \gamma) \mu^{2}-2[1+4 \gamma(\gamma-1)] \mu+2\left[1-\gamma\left(4 \gamma^{2}-8 \gamma+5\right)\right]}{\mu^{2}-2(1-2 \gamma) \mu-2(1-\gamma)}
$$

Now given parameters $(\gamma, \mu)$ we can determine $\rho$ and hence $\Omega$ from $\Omega=\rho(\mu-1)$. The $(\Omega, \mu)$ parameter space for $\Omega \geq 0, \mu \geq 0$ corresponds to $\gamma \leq 0.5$ and $\mu \geq 0$, as shown in Fig. 19. The curve $\gamma=0$ determines the Hopf bifurcation surface where a complex-conjugate pair of eigenvalues reaches the imaginary axis.

The center-manifold reduction for this bifurcation requires that we determine the two-dimensional center subspace. For the eigenvalues $\left(\lambda_{1}, \lambda_{2}, \lambda_{3}\right)$ we have eigenvectors $\left(v_{1}, v_{2}, v_{3}\right)$ :

$$
v_{i}=\left(\begin{array}{c}
-\lambda_{i} \rho(1+\mu) \\
-A\left(\lambda_{i}\right) \\
\rho \mu\left(1+\rho^{2}\right)(1+\mu)
\end{array}\right), \quad i=1,2,3,
$$

where $A(\lambda) \equiv \lambda^{2}-\lambda+\mu\left(1+\rho^{2}\right)$. For the real eigenvalue $\lambda_{1}$, the eigenvector is real; for the conjugate pair $\lambda_{2}=\bar{\lambda}_{3}$ we have

$$
v_{2,3}=v_{r} \pm i v_{i}
$$

$$
S^{-1}=\left[\frac{\beta}{\operatorname{det} S}\right]\left[\begin{array}{cc}
\alpha \omega(1-2 \gamma) & \alpha \beta \omega \\
-\alpha\left[\omega^{2}+A\left(\lambda_{1}\right)-A(\mu)\right] & \alpha \beta\left(\lambda_{1}-\gamma\right) \\
\alpha \omega(2 \gamma-1) & -\alpha \beta \omega
\end{array}\right.
$$

with

$$
\begin{aligned}
& \alpha \equiv \mu\left(1+\rho^{2}\right), \quad \beta \equiv \rho(1+\mu), \\
& \operatorname{det} S=\alpha \beta^{2} \omega\left[A\left(\lambda_{1}\right)-A(\gamma)+\omega^{2}+\left(\lambda_{1}-\gamma\right)(1-2 \gamma)\right] .
\end{aligned}
$$

Next we implement the linear change of variables

$$
\left(\begin{array}{l}
x^{\prime} \\
y^{\prime} \\
z^{\prime}
\end{array}\right)=S^{-1}\left(\begin{array}{l}
x \\
y \\
z
\end{array}\right)
$$

in Eq. (9.4), to express the vector field in the standard form of Eq. (7.2):

$\frac{d}{d t}\left[\begin{array}{l}x^{\prime} \\ y^{\prime}\end{array}\right]=\left[\begin{array}{cc}\gamma & \omega \\ -\omega & \gamma\end{array}\right]\left[\begin{array}{l}x^{\prime} \\ y^{\prime}\end{array}\right]+\left[\begin{array}{l}R_{1}\left(x^{\prime}, y^{\prime}, z^{\prime}\right) \\ R_{2}\left(x^{\prime}, y^{\prime}, z^{\prime}\right)\end{array}\right]$ where

$$
\begin{aligned}
& v_{r}=\left[\begin{array}{c}
-\gamma \rho(1+\mu) \\
\omega^{2}-A(\gamma) \\
\rho \mu\left(1+\rho^{2}\right)(1+\mu)
\end{array}\right], \\
& v_{i}=\omega\left(\begin{array}{c}
-\rho(1+\mu) \\
1-2 \omega \\
0
\end{array}\right)
\end{aligned}
$$

span $E^{c}$ at $\gamma=0$. The linear transformation

$$
S \equiv\left(\begin{array}{lll}
v_{r} & v_{i} & v_{1}
\end{array}\right)
$$

puts the linear problem in block-diagonal form:

$$
\left[\begin{array}{ccc}
\gamma & \omega & 0 \\
-\omega & \gamma & 0 \\
0 & 0 & \lambda_{1}
\end{array}\right]=S^{-1} L S
$$

where $L$ is the matrix in Eq. (9.4) and $S^{-1}$ is given by

$$
\left.\begin{array}{c}
\omega\left[A\left(\lambda_{1}\right)+\lambda_{1}(1-2 \gamma)\right] \\
\lambda_{1} A(\gamma)-\gamma A\left(\lambda_{1}\right)-\lambda_{1} \omega^{2} \\
\omega\left[\omega^{2}-A(\gamma)-\gamma(1-2 \gamma)\right]
\end{array}\right]
$$

$$
\frac{d z^{\prime}}{d t}=\lambda_{1} z^{\prime}+R_{3}\left(x^{\prime}, y^{\prime}, z^{\prime}\right)
$$

where

$$
\left[\begin{array}{l}
R_{1}\left(x^{\prime}, y^{\prime}, z^{\prime}\right) \\
R_{2}\left(x^{\prime}, y^{\prime}, z^{\prime}\right) \\
R_{3}\left(x^{\prime}, y^{\prime}, z^{\prime}\right)
\end{array}\right]=2 S^{-1} \cdot\left(\begin{array}{c}
-y^{2} \\
x y \\
-x z
\end{array}\right)
$$

with $(x, y, z)$ expressed in terms of $\left(x^{\prime}, y^{\prime}, z^{\prime}\right)$ using Eq. (9.14). For convenience in our discussion below, the result of fully expanding the right-hand side of Eq. (9.17) will be denoted

$$
\begin{aligned}
R_{i}\left(x^{\prime}, y^{\prime}, z^{\prime}\right)= & R_{i 1} x^{\prime 2}+R_{i 2} y^{\prime 2}+R_{i 3} x^{\prime} y^{\prime} \\
& +R_{i 4} x^{\prime} z^{\prime}+R_{i 5} y^{\prime} z^{\prime}+R_{i 6} z^{\prime 2}
\end{aligned}
$$


for each component $i=1,2,3$. The coefficients $R_{i j}$ are readily worked out, but we shall not require the detailed expressions, which tend to be unwieldy, e.g.,

$$
\begin{aligned}
R_{11}=\frac{-2 \alpha \beta \omega}{\operatorname{det} S}[ & (1-2 \gamma)\left(\omega^{2}-A(\gamma)\right)^{2} \\
& +\beta^{2} \gamma\left(\omega^{2}-A(\gamma)\right) \\
& \left.+\beta^{2} \gamma\left(A\left(\lambda_{1}\right)+\lambda_{1}(1-2 \gamma)\right)\right] .
\end{aligned}
$$

\section{Approximating the center manifold}

Near $\left(x^{\prime}, y^{\prime}, z^{\prime}\right)=(0,0,0)$ we represent the center manifold by a function $h: \mathbb{R}^{2} \rightarrow \mathbb{R}$ describing the $z^{\prime}$ coordinate of the manifold, i.e., $z^{\prime}=h\left(x^{\prime}, y^{\prime}\right)$. This function satisfies [cf. Eq. (7.7)]

$$
\begin{aligned}
& \frac{\partial h}{\partial x^{\prime}}\left[\gamma x^{\prime}+\omega y^{\prime}+R_{1}\left(x^{\prime}, y^{\prime}, h\right)\right] \\
& +\frac{\partial h}{\partial y^{\prime}}\left[-\omega x^{\prime}+\gamma y^{\prime}+R_{2}\left(x^{\prime}, y^{\prime}, h\right)\right] \\
& \quad=\lambda_{1} h\left(x^{\prime}, y^{\prime}\right)+R_{3}\left(x^{\prime}, y^{\prime}, h\right)
\end{aligned}
$$

with

$$
h(0,0)=0, \frac{\partial h}{\partial x^{\prime}}(0,0)=\frac{\partial h}{\partial y^{\prime}}(0,0)=0 .
$$

An asymptotic solution for $h\left(x^{\prime}, y^{\prime}\right)$ near $\left(x^{\prime}, y^{\prime}\right)=(0,0)$ has the form

$$
h\left(x^{\prime}, y^{\prime}\right)=h_{1} x^{\prime 2}+h_{2} y^{\prime 2}+h_{3} x^{\prime} y^{\prime}+\cdots
$$

where terms in $\left(x^{\prime}, y^{\prime}\right)$ of third degree or higher have been dropped. A straightforward evaluation of the quadratic coefficients yields

$$
\begin{aligned}
& h_{3}=\frac{2 \omega\left(R_{32}-R_{31}\right)+\left(2 \gamma-\lambda_{1}\right) R_{33}}{(2 \omega)^{2}+\left(2 \gamma-\lambda_{1}\right)^{2}}, \\
& h_{1}=\frac{\omega h_{3}+R_{31}}{2 \gamma-\lambda_{1}}, \\
& h_{2}=\frac{-\omega h_{3}+R_{32}}{2 \gamma-\lambda_{1}} .
\end{aligned}
$$

Given $h\left(x^{\prime}, y^{\prime}\right)$, the two-dimensional vector field on the center manifold follows directly from Eqs. (9.15), (9.18), and (9.21),

$$
\begin{aligned}
{\left[\begin{array}{l}
\dot{x}^{\prime} \\
\dot{y}^{\prime}
\end{array}\right]=} & {\left[\begin{array}{cc}
\gamma & \omega \\
-\omega & \gamma
\end{array}\right]\left[\begin{array}{l}
x^{\prime} \\
y^{\prime}
\end{array}\right]+\left[\begin{array}{l}
R_{1}\left(x^{\prime}, y^{\prime}, h\right) \\
R_{2}\left(x^{\prime}, y^{\prime}, h\right)
\end{array}\right] } \\
= & {\left[\begin{array}{cc}
\gamma & \omega \\
-\omega & \gamma
\end{array}\right]\left[\begin{array}{l}
x^{\prime} \\
y^{\prime}
\end{array}\right]+\left[\begin{array}{l}
R_{11} x^{\prime 2}+R_{12} y^{\prime 2}+R_{13} x^{\prime} y^{\prime} \\
R_{21} x^{\prime 2}+R_{22} y^{\prime 2}+R_{23} x^{\prime} y^{\prime}
\end{array}\right] } \\
& +\left(h_{1} x^{\prime 2}+h_{2} y^{\prime 2}+h_{3} x^{\prime} y^{\prime}\right)\left(\begin{array}{l}
R_{14} x^{\prime}+R_{15} y^{\prime} \\
R_{24} x^{\prime}+R_{25} y^{\prime}
\end{array}\right]+\cdots
\end{aligned}
$$

where terms of fourth degree and higher have been omitted.

\section{Determining the normal form}

The quadratic terms in Eq. (9.25) may be removed by a near-identity normal-form transformation to new variables $(x, y)=\left(x^{\prime}, y^{\prime}\right)+\phi^{(2)}\left(x^{\prime}, y^{\prime}\right) \quad$ with inverse $\left(x^{\prime}, y^{\prime}\right)=(x, y)-\phi^{(2)}(x, y)+\mathcal{O}(3)$ [cf. Eq. (8.3)]. From Eqs. (9.25) and (8.8), the equation for $\phi^{(2)}$ is

$$
L\left(\phi^{(2)}\right)=\left[\begin{array}{l}
R_{11} x^{2}+R_{12} y^{2}+R_{13} x y \\
R_{21} x^{2}+R_{22} y^{2}+R_{23} x y
\end{array}\right]
$$

where $L\left(\phi^{(2)}\right)$ is defined in Eq. (8.16). Following the discussion in VII.A.2, we solve Eq. (9.26) by rewriting it relative to the basis $\left\{\xi_{ \pm}^{(2, l)}\right\}$ defined in Eq. (8.19). Thus

$$
\left[\begin{array}{l}
R_{11} x^{2}+R_{12} y^{2}+R_{13} x y \\
R_{21} x^{2}+R_{22} y^{2}+R_{23} x y
\end{array}\right]=\sum_{l=0}^{2}\left[R_{+}^{(2, l)} \xi_{+}^{(2, l)}+R_{-}^{(2, l)} \xi_{-}^{(2, l)}\right]
$$

with

$$
\begin{aligned}
R_{+}^{(2,0)} & =\frac{1}{4}\left(R_{11}-R_{12}-R_{23}\right)+\frac{i}{4}\left(R_{13}+R_{21}-R_{22}\right) \\
& =\overline{R_{-}^{(2,2)}}, \\
R_{+}^{(2,1)} & =\frac{1}{2}\left(R_{11}+R_{12}\right)+\frac{i}{2}\left(R_{21}+R_{22}\right)=\overline{R_{-}^{(2,1)}}, \\
R_{+}^{(2,2)} & =\frac{1}{4}\left(R_{11}-R_{12}+R_{23}\right)+\frac{i}{4}\left(R_{21}-R_{13}-R_{22}\right) \\
& =\overline{R_{-}^{(2,0)}}
\end{aligned}
$$

and

$$
\phi^{(2)}=\sum_{l=0}^{2}\left[\phi_{+}^{(2, l)} \xi_{+}^{(2, l)}+\phi_{-}^{(2, l)} \xi_{-}^{(2, l)}\right] ;
$$

hence from Eqs. (8.22) and (9.26)

$$
\phi_{ \pm}^{(2, l)}=\frac{R_{ \pm}^{(2, l)}}{\lambda_{ \pm}^{(2, l)}}
$$

for $l=0,1,2$. This change of coordinates must now be carried out in Eq. (9.25) to obtain the transformed vector field up to terms of fourth degree:

$$
\begin{aligned}
{\left[\begin{array}{l}
\dot{x} \\
\dot{y}
\end{array}\right]=} & {\left[\begin{array}{cc}
\gamma & \omega \\
-\omega & \gamma
\end{array}\right]\left[\begin{array}{l}
x \\
y
\end{array}\right] } \\
& +\left(h_{1} x^{2}+h_{2} y^{2}+h_{3} x y\right)\left(\begin{array}{l}
R_{14} x+R_{15} y \\
R_{24} x+R_{25} y
\end{array}\right] \\
& -D \phi^{(2)}(x, y) \cdot\left[\begin{array}{l}
R_{11} x^{2}+R_{12} y^{2}+R_{13} x y \\
R_{21} x^{2}+R_{22} y^{2}+R_{23} x y
\end{array}\right]+\mathcal{O}(4) .
\end{aligned}
$$


Here we see the additional terms of third degree generated by the nonlinear coordinate change removing the quadratic terms. The final task is to consider the terms of third degree in Eq. (9.31) relative to the basis $\left\{\xi_{ \pm}^{(3, l)}\right\}$ and determine the coefficient $\alpha_{1}$ of $\xi_{+}^{(3,2)}$ [cf. Eq. (8.25)]. This calculation yields

$$
\begin{aligned}
\alpha_{1}= & R_{+}^{(3,2)}-\phi_{+}^{(2,1)}\left(R_{+}^{(2,2)}+R_{-}^{(2,1)}\right) \\
& -2\left(\phi_{+}^{(2,2)} R_{+}^{(2,1)}+\phi_{+}^{(2,0)} R_{-}^{(2,2)}\right),
\end{aligned}
$$

where $R_{+}^{(3,2)}$ is the component of the "original" cubic terms in Eq. (9.25) along the basis vector $\xi_{+}^{(3,2)}$,

$$
\begin{aligned}
R_{+}^{(3,2)=} \frac{1}{8}[ & 3\left(h_{1} R_{14}+h_{2} R_{25}\right)+h_{2} R_{14} \\
& \left.+h_{3} R_{15}+h_{1} R_{25}+h_{3} R_{24}\right] \\
+ & \frac{i}{8}\left[3\left(h_{1} R_{24}-h_{2} R_{14}\right)+h_{2} R_{24}+h_{3} R_{25}\right. \\
& \left.-h_{1} R_{15}-h_{3} R_{14}\right] .
\end{aligned}
$$

We now have the normal form for this bifurcation to leading nonlinear order [cf. Eq. (8.26)]:

$$
\begin{aligned}
& \dot{r}=\gamma r+\operatorname{Re}\left(\alpha_{1}\right) r^{3}+\mathcal{O}\left(r^{5}\right), \\
& \dot{\theta}=\omega-\operatorname{Im}\left(\alpha_{1}\right) r^{2}+\mathcal{O}\left(r^{4}\right) .
\end{aligned}
$$

The dependence of $\alpha_{1}$ on parameters is complicated, and the behavior of the cubic coefficient $\operatorname{Re} \alpha_{1}$ along the critical curve $\gamma=0$ in parameter space is best examined numerically. The graph of $a_{1} \equiv \operatorname{Re} \alpha_{1}$ vs $\mu$ for $\gamma=0$ in Fig. 20 indicates a region of supercritical bifurcation $a_{1}<0$ and a region of subcritical bifurcation $a_{1}>0$, with the transition $a_{1}=0$ occurring at $\mu_{c} \sim 3.29$. Thus for damping rates greater than $\mu_{c}$ the instability will saturate at $r=r_{H}$ in a small stable oscillation of the wave amplitudes [cf. Eq. (5.25)]. For $\mu<\mu_{c}$, the analysis implies that there

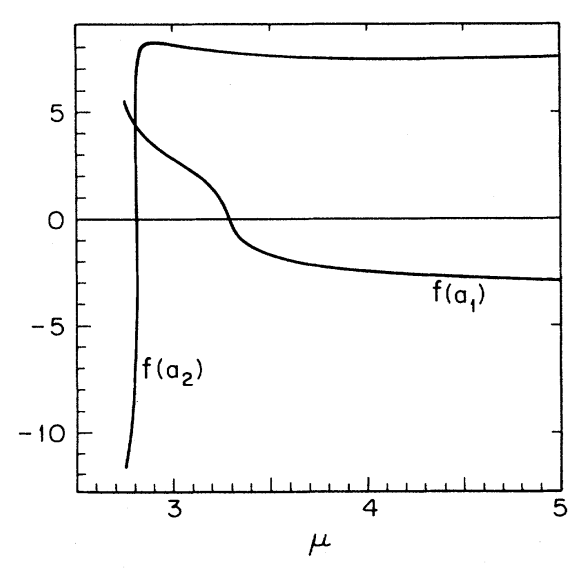

FIG. 20. At critically $(\gamma=0)$ the norinal-form coefficients $a_{1}=\operatorname{Re}\left(\alpha_{1}\right)$ and $a_{2}=\operatorname{Re}\left(\alpha_{2}\right)$ in Eq. (9.34a) are plotted against $\mu$ using the function $f(x) \equiv \operatorname{sgn}(x) \log (1.0+|x|)$. is no stable solution in the neighborhood of $r=0$ when $\gamma$ is positive; in fact, numerical studies indicate that the wave amplitudes grow without bound.

These conclusions indicate that the stable Hopf periodic orbit must be destroyed in a separate bifurcation in the parameter neighborhood of $\left(\mu=\mu_{c}, \gamma=0\right)$, since there is no periodic orbit in the neighborhood of the fixed point for $\mu \leq \mu_{c}, 0<\gamma<<1$. Thus in parameter space the curve or bifurcation surface at $\gamma=0$ corresponding to Hopf bifurcation must intersect at least one additional such curve at $\left(\mu=\mu_{c}, \gamma=0\right)$. The instability of $r=0$ at this point is termed a degenerate Hopf bifurcation because the nondegeneracy condition (5.24) fails, and the discovery of additional bifurcations at this point illustrates the value of analyzing such degenerate cases. This particular degeneracy is one of the simplest examples of a codimension-two bifurcation, meaning that to locate it we must simultaneously adjust two independent parameters $\mu$ and $\gamma$.

The comprehensive analysis of degenerate Hopf bifurcation by Golubitsky and Langford (1981) shows that in this case there is only one additional bifurcation surface that intersects the Hopf surface. This second surface marks parameters values at which the stable Hopf periodic orbit merges with an unstable periodic orbit and both disappear. In the return map for the Hopf orbit, this merger is a saddle-node bifurcation which annihilates two fixed points. For this reason, this second surface may be referred to as the saddle-node (SN) surface; it was discovered numerically by Meunier et al. (1982).

To determine how the $S N$ surface approaches the Hopf surface requires an analysis that includes both periodic orbits. Since the $S N$ surface intersects the Hopf surface at $\gamma=0$, the saddle-node bifurcation occurs for arbitrarily small positive values of $\gamma$. This means that the two orbits can merge while the Hopf orbit is still in a very small neighborhood of $r=0$. Under these circumstances the local attractivity of $W^{c}$ near $r=0$ will not permit a periodic orbit that is not in fact contained in $W^{c}$. Hence both periodic orbits must lie in $W^{c}$ and their merger is a feature of the center-manifold dynamics (9.34). Since the phase-shift symmetry decouples $\dot{\theta}$ from $\dot{r}$, the radial equation (9.34a) is adequate to describe the saddle-node bifurcation provided the fifth-order term $a_{2} r^{5}$ is included.

At criticality for the saddle-node bifurcation the linear stability of the Hopf orbit is lost, but the orbit still exists. Near $\gamma=0$, the $S N$ surface is determined by these two facts. The existence of the Hopf orbit at criticality means that $r=r_{H}$ is still a solution to $d r / d t=0$, which implies

$$
\gamma+a_{1} r_{H}^{2}+a_{2} r_{H}^{4}=0
$$

In addition, linearizing Eq. (9.34a) about the Hopf orbit determines the orbit's linear stability within $W^{c}$, setting $\eta=r-r_{H}$ we find

$$
\frac{d \eta}{d t}=\left(\gamma+3 a_{1} r_{H}^{2}+5 a_{2} r_{H}^{4}\right) \eta+\mathcal{O}\left(\eta^{2}\right)
$$

Linear stability of $\eta=0$ changes when 


$$
\gamma+3 a_{1} r_{H}^{2}+5 a_{2} r_{H}^{4}=0 .
$$

Equations (9.35) and (9.36) suffice to determine the $S N$ surface at small $\gamma$. Subtracting (9.35) from (9.36) yields $r_{H}^{2}\left(2 a_{2} r_{H}^{2}+a_{1}\right)=0$, hence $r_{H}^{2}>0$ requires

$$
\frac{a_{1}}{a_{2}}<0
$$

for a valid solution $r_{H}^{2}=-a_{1} / 2 a_{2}$ to exist. Substituting this solution into Eq. (9.35) or (9.36) yields

$$
4 \gamma=\frac{a_{1}^{2}}{a_{2}} \text {. }
$$

Taken together relations (9.37) and (9.38) locate the $S N$ bifurcation surface for $0 \leq \gamma<<1$. There are two cases, depending on the sign of $a_{2}$ at $\left(\mu=\mu_{c}, \gamma=0\right)$. From the point of degeneracy the saddle-node surface branches to the right $\left(a_{1}<0\right)$ if $a_{2}>0$ and to the left $\left(a_{1}>0\right)$ if $a_{2}<0$. These cases are indicated in Fig. 21.

The actual calculation of $a_{2}$ is a straightforward extension of the calculation of $a_{1}$. The calculation of $h(x, y)$ must be carried to fourth order so that Eq. (9.25) can be extended to include fifth-order terms. Then second-, third-, and fourth-degree terms need to be removed to
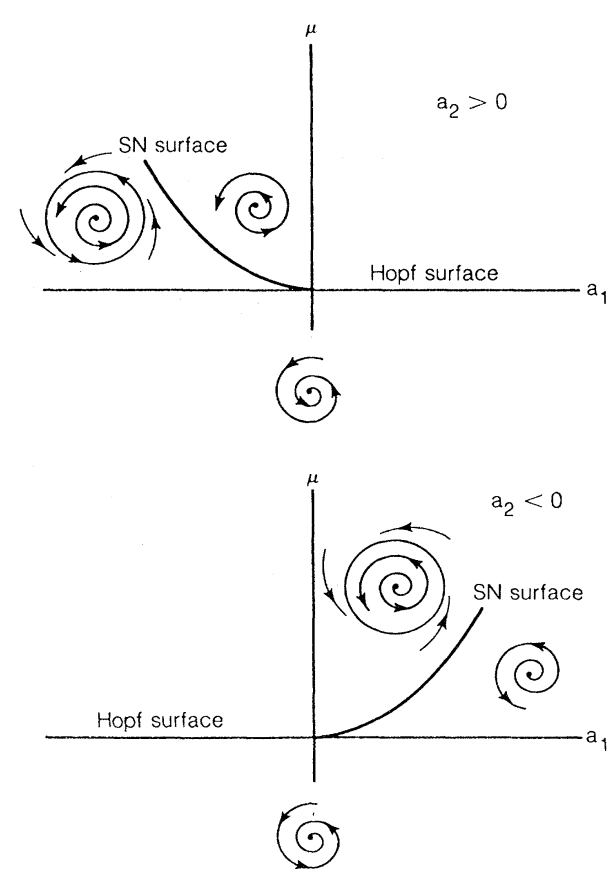

FIG. 21. For the degenerate Hopf bifurcation corresponding to $a_{1}=0$ and $a_{2} \neq 0$ there are two possibilities, depending on the sign of $a_{2}$ at criticality. For $a_{2}>0$, the saddle-node (SN) surface branches toward negative values of $a_{1}$. For $a_{2}<0$, the $S N$ surface branches toward positive values of $a_{1}$. The unstable periodic orbit which collides with the stable Hopf orbit is not shown. obtain the normal form through fifth order. The details of this are not of interest here; the resulting expression for $a_{2}$ as a function of $\mu$ for $\gamma=0$ is also plotted in Fig. 20. At the degenerate Hopf point $\left(a_{1}=0\right)$ we find $a_{2}>0$ and conclude that the saddle-node surface branches to the right.

The results of this bifurcation analysis may be tested numerically. Figure 22 shows the Hopf bifurcation to a stable oscillation for $\mu>\mu_{c}$. As $\mu$ decreases at fixed $\gamma=0.01$, the stable periodic orbit loses stability near $\mu \sim 3.55$. This transition appears in Fig. 23 and reveals the dramatic effect of the saddle-node bifurcation.

\section{B. Steady-state bifurcation \\ in the Ginzburg-Landau equation}

For the complex-valued function $A(x, \tau)$ we consider the Ginzburg-Landau (GL) equation in one space dimension,
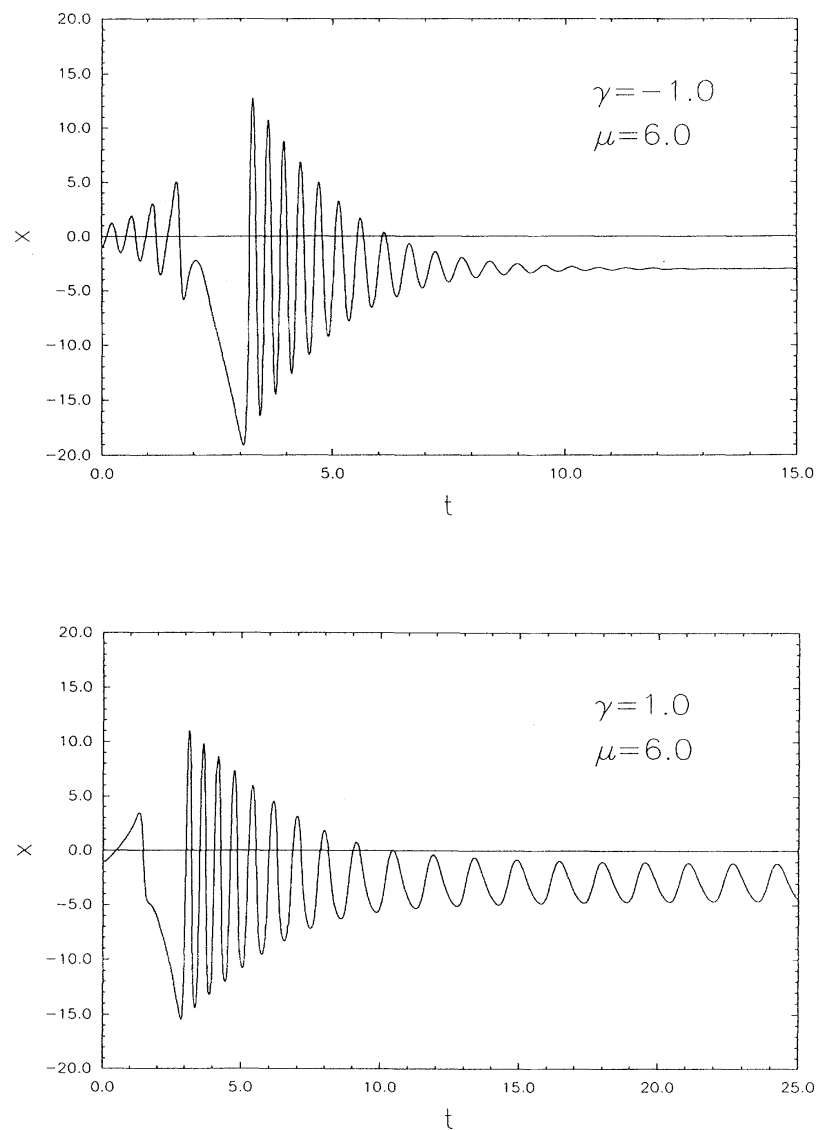

FIG. 22. Evolution of $x(t)$ vs $t$ for Eq. (9.1) from an initial condition $(-1.0,0.0,0.5)$ : (a) With $\gamma=-0.1$ and $\mu=6.0$ when the fixed point (9.3) is stable; note that the trajectory is initially repelled from the unstable fixed point at the origin. (b) With $\gamma=0.1$ and $\mu=6.0$ when the fixed point is unstable and the solution is attracted to the stable Hopf periodic orbit. The final point on this trajectory segment was $(-4.486,-2.886,4.499)$. 


$$
\frac{\partial A}{\partial \tau}=\mu A+\frac{\partial^{2} A}{\partial x^{2}}-A|A|^{2},
$$

with real coefficients and with boundary conditions that ensure finite-dimensional center manifolds (Tuckerman and Barkley, 1990). This equation arises in a wide variety of settings; in particular, Eq. (9.39) models the behavior of a spatially extended system near criticality for a steady-state bifurcation (Collet and Eckmann, 1990; Manneville, 1990). In fluid dynamics, a well-studied example of such a bifurcation is the appearance of Taylor vortex flow in a Taylor-Couette apparatus (Ahlers et al., 1986; Ahlers, 1989), where one observes the motion of a fluid confined in the gap between two concentric cylinders. Taylor's original investigation (1923), in which he rotated the inner cylinder with frequency $\Omega$ and fixed the outer cylinder, established a critical frequency $\Omega_{c}$ above which the steady and (nearly) featureless flow develops a pattern of vortices characterized by a welldefined axial wave number $q$. The fluid mechanisms that
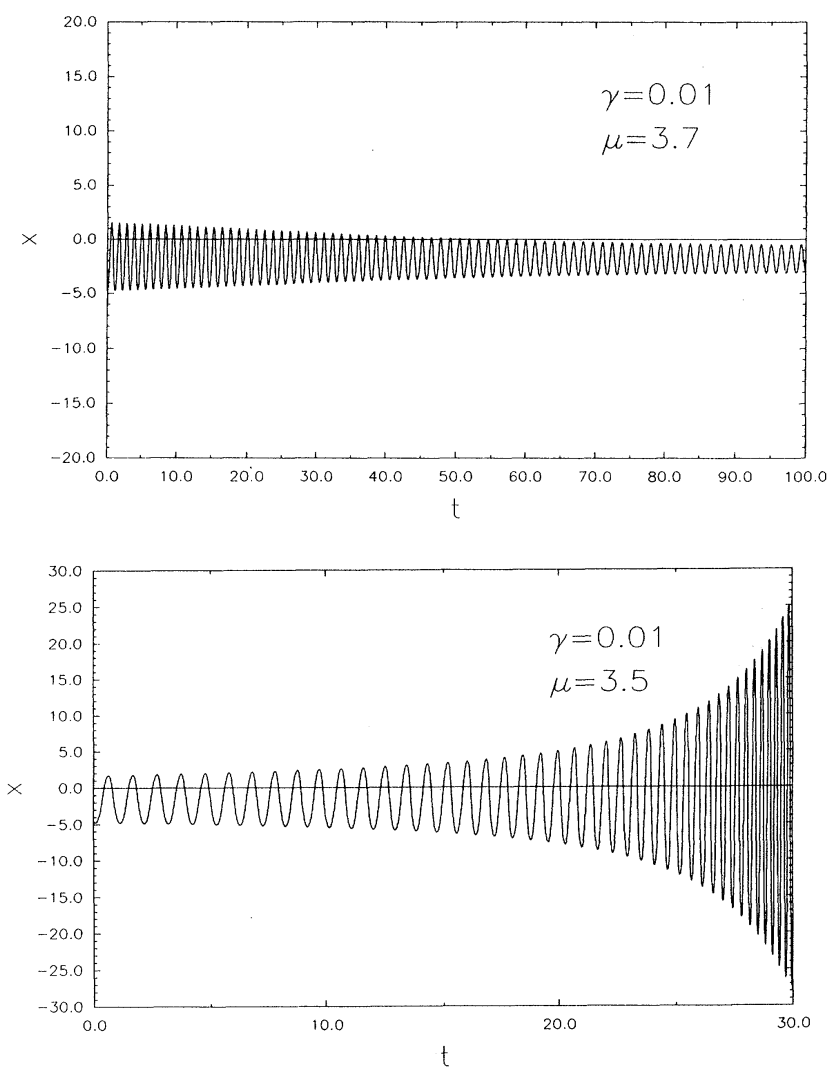

FIG. 23. Evolution of $x(t)$ vs $t$ for Eq. (9.1) with the final point given for Fig. 22(b) used as the initial condition: (a) for $\gamma=0.01$ and $\mu=3.7$ when the Hopf periodic orbit is stable; (b) for the same initial condition with $\gamma=0.01$ and $\mu=3.5$, after the Hopf periodic orbit has been destroyed. No stable orbit remains and the solution grows without bound. Notice the difference in the vertical scale. determine the wavelength $2 \pi / q$ of the vortex flow have been carefully investigated as a particularly simple paradigm for nonequilibrium pattern formation (Langer, 1986; Ahlers, 1989).

Analytic theory often assumes either cylinders of infinite length or periodic axial boundary conditions. ${ }^{28}$ Then in linear approximation one finds an eigenfunction with axial wave number $q_{c}$ whose (real) eigenvalue approaches zero as $\Omega$ tends to $\Omega_{c}$ from below. Slightly above this threshold all wave numbers within a band of width $\sqrt{\mu}$ about $q_{c}$ are linearly unstable, where $\mu \equiv\left(\Omega-\Omega_{c}\right) / \Omega_{c}$ defines the bifurcation parameter. However, only those wave numbers within a subband of width $\mu$ are actually realized experimentally because of a secondary instability that arises for $q$ values outside the subband. This latter instability is known as the Eckhaus instability and it modifies $q$ by adding or subtracting vortex pairs.

The competition between different linearly unstable wavelengths can be studied near criticality $(0<\mu<1)$ by developing the fluid equations in an expansion in $\mu$. How this expansion leads to the GL equation can be briefly sketched by avoiding the complexity of a realistic model and assuming that the system is described by a single field $u(z, t)$ such that $u \equiv 0$ corresponds to the featureless equilibrium. For small $\mu$, one defines rescaled space and time variables by $x=\sqrt{\mu z}$ and $\tau=\mu t$ and seeks solutions of the form

$$
u(z, t)=\mu u_{0}(z, x, \tau)+\mu^{2} u_{1}(z, x, \tau)+\cdots,
$$

which are independent of the fast time scale $t$ and describe the slow evolution of the pattern about the basic length scale $q_{c}^{-1}$. The leading-order balance determines the form of $u_{0}$,

$$
u_{0}(z, x, \tau)=A(x, \tau) e^{i q_{c} z}+\text { c.c. },
$$

in terms of a complex amplitude function; at higher order the GL equation (9.39) for $A$ arises as a "solvability" condition, which must be satisfied to avoid secular behavior. The basic equilibrium $A=0$ for GL corresponds, therefore, to the spatially uniform state one observes if $\mu<0$; in addition, for $\mu>0$ there are spatially periodic equilibria ("pure modes")

$$
A_{Q}(x ; \phi)=\sqrt{\mu-Q^{2}} e^{i \phi} e^{i Q x}
$$

that describe patterns with wave number $q=q_{c}+\sqrt{\mu} Q$.

As $\mu$ varies there are bifurcations from $A=0$ and $A_{Q}$ that can be studied using center-manifold theory; however, this analysis is more subtle for two reasons. First, the GL equation (9.39) is highly symmetric. The group of symmetries is generated by reflections and translations in

\footnotetext{
${ }^{28}$ There has also been interesting recent work on the necessity of allowing for finite end effects in order to describe some features of the experiments in long cylinders (Edwards, 1990).
} 
$x$, complex conjugation, and phase shifts; these operations we denote by $\kappa, T_{d}, C$, and $R_{\theta}$, respectively:

$$
\begin{aligned}
& (\kappa \cdot A)(x)=A(-x), \\
& \left(T_{d} \cdot A\right)(x)=A(x+d), \\
& (C \cdot A)(x)=\overline{A(x)} \\
& \left(R_{\theta} \cdot A\right)(x)=e^{i \theta} A(x)
\end{aligned}
$$

Thus if $A(x, t)$ is a solution then $(\gamma \cdot A)(x, t)$ is also a solution for $\gamma=\kappa, T_{d}, C, R_{\theta}$, or any combination of these operations. For bifurcation problems with symmetry there exists a generalization of theory presented in Secs. II-VIII that incorporates a variety of group-theoretic techniques. We do not require this generalization for this example, but we will indicate how the symmetry (9.43) affects the bifurcation analysis. Golubitsky, Stewart, and Schaeffer (1988) provide a comprehensive introduction to equivariant bifurcation theory, and there are also the more concise reviews by Stewart (1988), Gaeta (1990), and Crawford and Knobloch (1991). A second novelty arises because Eq. (9.39) describes an infinite-dimensional dynamical system; i.e., it defines a flow on an infinitedimensional phase space-the space of functions $A(x)$. Center-manifold theory can be rigorously extended to partial differential equations, but this generalization is rather technical for the present discussion (see the recent review by Vanderbauwhede and Iooss, 1991). However, if we assume there are center manifolds associated with the bifurcations in Eq. (9.39), then the corresponding reduction and bifurcation analysis can be carried through just as for an ordinary differential equation.

The assumption of a finite-dimensional center manifold requires a consideration of boundary conditions for $\mathrm{Eq}$. (9.39). This necessity is clear if we analyze the linear stability of $A=0$. Linearizing Eq. (9.39) defines the operator $L$,

$$
\frac{\partial}{\partial t} A=\mu A+\frac{\partial^{2}}{\partial x^{2}} A \equiv L A,
$$

with eigenvectors and eigenvalues given by

$$
\psi_{\lambda}(x)=e^{i Q x}, \lambda=\mu-Q^{2},
$$

where $-\infty<Q<\infty$. For $\mu<0$ all eigenvalues satisfy $\lambda<0$, and the uniform state is asymptotically stable; for $\mu>0$ there is a continuous band of wave numbers $0<Q^{2}<\mu$ whose eigenvectors $\psi_{\lambda}$ describe linearly unstable perturbations of $A=0$. This continuum prevents us from isolating a finite number of critical modes that determine the time-asymptotic behavior and represents a serious technical obstacle to center-manifold reduction (Coullet and Spiegel, 1987). This difficulty does not arise when boundary conditions are imposed on $A(x)$ such that the eigenvalue spectrum becomes discrete. ${ }^{29}$ The periodic boundary condition

$$
A(-\pi)=A(\pi)
$$

is a simple choice that enforces the discretization $Q=$ integer in (9.45) and also respects the full set of symmetries (9.43), allowing us to observe their effects on the bifurcations. $^{30}$ With periodic boundary conditions the translations $T_{d}$ act as rotations on the periodic coordinate $x$. Consequently $T_{d}$ and $\kappa$ generate the symmetries of the circle; i.e., the group $O(2)$. In addition, $R_{\theta}$ and $C$ generate a second $O(2)$ action on the phase of $A(x)$, since these two $O(2)$ actions commute the full symmetry of Eq. (9.39) and Eq. (9.46) is $O(2) \times O(2)$.

\section{Bifurcation from $A=0$}

As $\mu$ increases through $\mu=Q^{2}, Q^{2}=0,1,4,9, \ldots$, the eigenvalue $\lambda=\mu-Q^{2}$ crosses zero and the linear mode $\psi_{\lambda}$ becomes unstable. Because of the symmetry there is in fact a four-dimensional center manifold associated with this instability. If we rewrite Eq. (9.44) in terms of real and imaginary parts, $\psi_{\lambda}=u(x)+i v(x)$, then $L \psi_{\lambda}=\lambda \psi_{\lambda}$ becomes

$$
\left(\mu+\frac{\partial^{2}}{\partial x^{2}}\right]\left[\begin{array}{l}
u \\
v
\end{array}\right]=\lambda\left(\begin{array}{l}
u \\
v
\end{array}\right)
$$

and for fixed $Q \neq 0$ there are four linearly independent real-valued eigenfunctions with eigenvalue $\lambda=\mu-Q^{2}$ :

$$
\begin{aligned}
& \psi_{1}(x)=\left[\begin{array}{l}
1 \\
0
\end{array}\right] \cos Q x, \\
& \psi_{2}(x)=R_{\pi / 2} \cdot \psi_{1}(x)=\left[\begin{array}{l}
0 \\
1
\end{array}\right] \cos Q x, \\
& \psi_{3}(x)=T_{-\pi / 2 Q} \cdot \psi_{1}(x)=\left[\begin{array}{l}
1 \\
0
\end{array}\right] \sin Q x, \\
& \psi_{4}(x)=T_{-\pi / 2 Q} R_{\pi / 2} \cdot \psi_{1}(x)=\left[\begin{array}{l}
0 \\
1
\end{array}\right] \sin Q x .
\end{aligned}
$$

The three "extra" eigenvectors $\psi_{2}, \psi_{3}$, and $\psi_{4}$ are forced by symmetry; thus the steady-state bifurcation at $\mu=Q^{2}$ involves a four-dimensional center subspace. The bifur-

\footnotetext{
${ }^{29}$ The contrast between a finite set of critical modes and a continuum of critical modes is not merely a matter of technical difficulty. In spatially extended systems one finds a rich variety of new phenomena (Brand, 1989; Collet and Eckmann, 1990; Manneville, 1990).

${ }^{30}$ Other boundary conditions have been considered in the literature; see for example Hall, 1980; Graham and Domaradzki, 1982; Ahlers et al., 1986; Tuckerman and Barkley, 1990.
} 
cation at $\mu=0$ when $Q=0$ is an exception, with only a two-dimensional center subspace: $E^{c}=\operatorname{span}\left\{\psi_{1}, \psi_{2}\right\}$ since $\psi_{1}=\psi_{3}$ and $\psi_{2}=\psi_{4}$. Returning to complex notation and introducing two complex amplitudes $(\alpha, \beta)$ for the critical modes (9.48), we have

$$
A(x, t)=\alpha(t) e^{i Q x}+\beta(t) e^{-i Q x}+S(x, t),
$$

where $S(x, t)$ is orthogonal to the critical modes

$$
\int_{-\pi}^{\pi} d x e^{ \pm i Q x} S(x, t)=0 .
$$

Note that the decomposition in Eq. (9.49) corresponds to the choice of variables in Eq. (7.2), with $(\alpha, \beta)$ corresponding to $x_{1}$ and $S$ corresponding to $x_{2}$. Near $A=0$, the center manifold for Eq. (9.49) may be represented as the graph of a function $h: E^{c} \rightarrow E^{s} \oplus E^{u}$; i.e.,

$$
S(x, t)=h(x, \alpha, \beta, \bar{\alpha}, \bar{\beta})
$$

such that

$$
h(x, 0,0)=0
$$

and

$$
\begin{aligned}
& \left.\frac{\partial}{\partial \alpha} h\right|_{(\alpha, \beta)=0}=0,\left.\frac{\partial h}{\partial \bar{\alpha}}\right|_{(\alpha, \beta)=0}=0, \\
& \left.\frac{\partial h}{\partial \beta}\right|_{(\alpha, \beta)=0}=0,\left.\frac{\partial h}{\partial \bar{\beta}}\right|_{(\alpha, \beta)=0}=0 .
\end{aligned}
$$

This exactly parallels the representation for $W^{c}$ introduced in Eq. (7.3), modified only by the fact that $E^{s} \oplus E^{u}$ is now infinite dimensional, i.e., the function $h$ depends on the continuous index $x$.

The reduced equations for the center-manifold dynamics depend on whether $Q=0$ or $Q \neq 0$ because the manifold dimension changes from four to two when $Q=0$.
a. $Q=0$

In this case the decomposition in Eq. (9.49) can be simplified to

$$
A(x, t)=\alpha(t)+S(x, t)
$$

where

$$
\int_{-\pi}^{\pi} d x S(x, t)=0 .
$$

In addition one finds from Eq. (9.39) that if $\partial A / \partial x=0$ at $t=0$, then the solution of (9.39) is independent of $x$ for all $t$; hence the center subspace is invariant under the full nonlinear dynamics, which implies that $E^{c}$ and $W^{c}$ coincide. Consequently $S(x, t)=0$ for solutions in the center manifold, and the dynamics on $W^{c}$ follows immediately by setting $S=0$ and inserting Eq. (9.54a) into (9.39):

$$
\frac{d}{d t} \alpha=\mu \alpha-|\alpha|^{2} \alpha
$$

As $\mu$ varies near $\mu=0$, this equation describes the bifurcation of the pure modes with zero wave number (9.42),

$$
A_{Q=0}=\sqrt{\mu} e^{i \phi} \text {. }
$$

Since the unstable subspace is empty at this bifurcation, these solutions are stable in the directions transverse to the center manifold. In addition, linearizing Eq. (9.55) around $\alpha=\sqrt{\mu} e^{i \phi}$ shows that these solutions are stable to perturbations in the amplitude $\left|A_{Q=0}\right|$ but that perturbations in the phase $\phi$ correspond to a zero eigenvalue or neutral linear stability. This zero eigenvalue reflects the fact that Eq. (9.56) describes a continuous family of equilibria parametrized by the phase $\phi$; such eigenvalues are a general feature of bifurcations that break a continuous symmetry. In this case, the $O(2)$ symmetry generated by $R_{\theta}$ and $C$ has been broken.

\section{b. $Q \neq 0$}

This bifurcation is only slightly more complicated, since the nonlinearity of the center manifold does not affect the lowest-order nonlinear terms in $\dot{\alpha}$ and $\dot{\beta}$. By inserting Eq. (9.50) into the GL equation and projecting with $\int_{-\pi}^{\pi} d x e^{ \pm i Q x}$ we obtain a system of equations analogous to $(7.2)$ :

$$
\begin{aligned}
& \dot{\alpha}=\left(\mu-Q^{2}\right) \alpha-\int_{-\pi}^{\pi} \frac{d x}{2 \pi} e^{-i Q x} A|A|^{2}, \\
& \dot{\beta}=\left(\mu-Q^{2}\right) \beta-\int_{-\infty}^{\pi} \frac{d x}{2 \pi} e^{i Q x} A|A|^{2}, \\
& \partial_{t} S=\left(\mu+\partial_{x}^{2}\right) S-A|A|^{2}+e^{i Q x} \int_{-\pi}^{\pi} \frac{d x}{2 \pi} e^{-i Q x} A|A|^{2} \\
& \quad+e^{-i Q x} \int_{-\pi}^{\pi} \frac{d x}{2 \pi} e^{i Q x} A|A|^{2}
\end{aligned}
$$

On the center manifold near $A=0, S$ is expressible in terms of $h$ [Eq. (9.51)], which is at least second order in the critical amplitudes as $|\alpha|,|\beta| \rightarrow 0$ [cf. Eqs. (9.52) and (9.53)]. Hence, on the center manifold,

$$
\begin{aligned}
A|A|^{2}= & \left(\alpha e^{i Q x}+\beta e^{-i Q x}\right) \\
& \times\left[|\alpha|^{2}+|\beta|^{2}+\alpha \bar{\beta} e^{+i 2 Q x}+\beta \bar{\alpha} e^{-i 2 Q x}\right]+O(4),
\end{aligned}
$$

where $O(4)$ denotes terms of fourth degree or higher in $(\alpha, \beta)$. Since the cubic terms (9.58) do not involve $h$ it will not be necessary to calculate the leading coefficients in its Taylor expansion. combining Eq. (9.58) with (9.57a) and (9.57b) yields the center-manifold equations to third order:

$$
\begin{aligned}
& \dot{\alpha}=\left(\mu-Q^{2}\right) \alpha-\left(2|\beta|^{2}+|\alpha|^{2}\right) \alpha+\mathcal{O}(5), \\
& \dot{\beta}=\left(\mu-Q^{2}\right) \beta-\left(2|\alpha|^{2}+|\beta|^{2}\right) \beta+\mathcal{O}(5) .
\end{aligned}
$$

In this four-dimensional system there is no longer any coupling to $S(x, t)$, and the neglected terms on the righthand side are at least fifth order in $(\alpha, \beta)$ because the first 
nonzero terms in the expansion of $h$ would appear at third order in this case rather than second order. Introducing polar variables $\alpha=\rho_{1} e^{i \psi_{1}}$ and $\beta=\rho_{2} e^{i \psi_{2}}$, we find that Eq. (9.59) reduces to a two-dimensional system,

$$
\begin{aligned}
& \dot{\rho}_{1}=\left(\mu-Q^{2}\right) \rho_{1}-\left(2 \rho_{2}^{2}+\rho_{1}^{2}\right) \rho_{1}+O(5), \\
& \dot{\rho}_{2}=\left(\mu-Q^{2}\right) \rho_{2}-\left(2 \rho_{1}^{2}+\rho_{2}^{2}\right) \rho_{2}+O(5), \\
& \dot{\psi}_{1,2}=0,
\end{aligned}
$$

since the amplitudes $\rho_{1,2}$ decouple from the phases $\psi_{1,2}$. One can show that this decoupling is a feature of the symmetry and extends to all orders; this, however, requires an analysis of the full normal form for this bifurcation; see Golubitsky, Stewart, and Schaeffer (1988), Chapter XVII, Sec. 2 for a relevant discussion.

The bifurcating solutions are obtained by setting $\dot{\rho}_{1}=\dot{\rho}_{2}=0$ and neglecting higher-order terms:

$$
\begin{aligned}
& \rho_{1}\left[\left(\mu-Q^{2}\right)-\left(2 \rho_{2}^{2}-\rho_{1}^{2}\right)\right]=0, \\
& \rho_{2}\left[\left(\mu-Q^{2}\right)-\left(2 \rho_{1}^{2}+\rho_{2}^{2}\right)\right]=0,
\end{aligned}
$$

There are two types of new solutions in addition to the trivial equilibrium at $(0,0)$. The pure-mode solutions, $\left(\rho_{1}^{2}=\mu-Q^{2}, \rho_{2}=0\right)$ and $\left(\rho_{1}=0, \rho_{2}^{2}=\mu-Q^{2}\right)$, correspond to the bifurcation of the states $A_{Q}$ and $A_{-Q}$, respectively, for $\mu-Q^{2}>0$. In addition there is a "mixed"-mode solution given by $\rho_{1}=\rho_{2}=\rho$ and $\rho_{2}=\frac{1}{3}\left(\mu-Q^{2}\right)$.

From Eq. (9.60) the linear stabilities within the center manifold of each of these states may be calculated. With respect to perturbations in the amplitudes $\left(\rho_{1}, \rho_{2}\right)$, the pure modes are stable but the mixed mode is unstable; in each case there are also two zero eigenvalues corresponding to perturbations in the phases $(9.60 \mathrm{c})$. The phase portrait Fig. 24 for $\left(\rho_{1}, \rho_{2}\right)$ summarizes this analysis.

In addition, for all of these solutions (pure and mixed modes) there can be unstable directions transverse to the center manifold because of the unstable directions for $A=0$. The number of these unstable directions is equal to the dimension of $E^{u}$ at criticality (see Table I). We shall see that these initially unstable pure modes $A_{Q}$ regain their stability as $\mu$ increases further above $\mu=Q^{2}$.

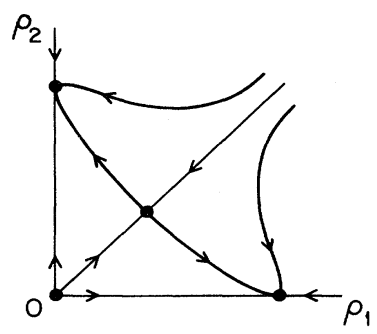

FIG. 24. Phase diagram for the flow on the center manifold associated with bifurcation from $A=0$. The pure modes are the stable fixed points on the $\rho_{1}$ axis and the $\rho_{2}$ axis. The unstable mixed mode lies on the diagonal.
TABLE I. Dimension of the unstable subspace for $A=0$ as a function of $\mu$.

\begin{tabular}{cc}
\hline \hline Value of $\mu$ & $\operatorname{dim} E^{u}$ \\
\hline$\mu \leq 0$ & 0 \\
$0<\mu \leq 1$ & 2 \\
$Q^{2}<\mu \leq(Q+1)^{2}, Q=1,2,3, \ldots$ & $2+4 Q$ \\
\hline \hline
\end{tabular}

\section{A digression on phase dynamics}

It is instructive to analyze the stability of these equilibria by introducing the phase and amplitude of $A$ and defining a local time-dependent wave number $k(x, \tau)$,

$$
\begin{aligned}
& A(x, \tau)=\rho(x, \tau) e^{i \psi(x, \tau)} \\
& k(x, \tau)=\frac{\partial \psi}{\partial x}
\end{aligned}
$$

In these variables, Eq. (9.39) becomes

$$
\begin{aligned}
& \frac{\partial \rho}{\partial \tau}=\left(\mu-k^{2}\right) \rho+\frac{\partial^{2} \rho}{\partial x^{2}}-\rho^{3} \\
& \frac{\partial k}{\partial \tau}=\frac{\partial}{\partial x}\left[\frac{2 k}{\rho} \frac{\partial \rho}{\partial x}+\frac{\partial k}{\partial x}\right] .
\end{aligned}
$$

If we restrict our attention to solutions that are slowly varying in $x$, then for $\mu>k^{2}$ we expect $\rho(x, \tau)$ to approach a quasistatic equilibrium

$$
\rho(x, \tau) \approx \sqrt{\mu-k^{2}(x, \tau)},
$$

since the $\partial^{2} \rho / \partial x^{2}$ term in Eq. (9.63a) can be neglected and $k(x, \tau)$ evolves on a time scale set by the slowly varying $x$ dependence. When $\rho$ is given by Eq. (9.64), the equation (9.63b) for the wave number reduces to a nonlinear diffusion equation,

$$
\frac{\partial k}{\partial \tau}=\frac{\partial}{\partial x}\left[D(k) \frac{\partial}{\partial x} k(x)\right]
$$

with diffusivity $D(k)$ given by

$$
D(k)=\frac{\mu-3 k^{2}}{\mu-k^{2}} .
$$

For a pure mode with $\rho=\sqrt{\mu-Q^{2}}$ and $k=Q$, consider the dynamics of a small fluctuation in phase, $k(x, \tau)=Q+\delta Q(x, \tau)$ [relaxing the periodic boundary condition (9.46)]. Inserting this ansatz into Eq. (9.65a) and linearizing in $\delta Q$ yields a linear diffusion equation for the fluctuation

$$
\frac{\partial}{\partial \tau} \delta Q=D(Q) \frac{\partial^{2}}{\partial x^{2}} \delta Q
$$

If the wavelength of the pure mode $q=q_{c}+Q$ satisfies

$$
Q^{2}<\mu / 3
$$

then $D(Q) \geq 0$ and $\delta Q$ decays, the pure mode is stable. 
The loss of phase stability when $Q^{2}>\mu / 3$ is known as the Eckhaus instability; its physical interpretation as negative phase diffusion was suggested by Pomeau and Manneville (1979). Subsequently more general theories of phase dynamics have been developed (Cross and Newell, 1984; Brand; 1988; Newell et al., 1990).

\section{Bifurcation from the pure modes}

We now develop the bifurcation theory of this instability with the periodic boundary condition (9.46).

\section{a. Symmetry}

The $O(2) \times O(2)$ symmetry of the $A=0$ equilibrium is partially broken in the pure-mode state; to describe the remaining symmetry define composite "translation" and "reflection" transformations $\widetilde{T}_{d}$ and $\widetilde{\kappa}$ by

$$
\begin{aligned}
& \widetilde{T}_{d}(Q) \equiv R_{-Q d} T_{d}, \\
& \widetilde{\kappa}(\phi) \equiv R_{\phi} C_{\kappa} R_{-\phi} .
\end{aligned}
$$

These transformations generate a representation of $O(2)$, which we denote by $\widetilde{O}(2)$, and the $A_{Q}(x ; \phi)$ state is invariant with respect to this $\widetilde{O}(2)$ action:

$$
\gamma \cdot A_{Q}(x ; \phi)=A_{Q}(x ; \phi), \quad \gamma=\widetilde{T}_{d}(Q), \widetilde{\kappa}(\phi) .
$$

In addition, the discrete group $Z_{Q}$ generated by $2 \pi / Q$ spatial translations $\left(T_{2 \pi / Q}\right)$ is a symmetry of $A_{Q}(x ; \phi)$.

Let $a(x, \tau)$ be defined by

$$
A(x, \tau)=A_{Q}(x ; \phi)(1+a(x, \tau)) .
$$

Then Eq. (9.39) implies

$$
\frac{\partial}{\partial \tau} a=\mathcal{L} a-\left(\mu-Q^{2}\right) N(a, \bar{a}),
$$

where

$\mathcal{L} a \equiv \frac{\partial^{2} a}{\partial x^{2}}+2 i Q \frac{\partial a}{\partial x}-\left(\mu-Q^{2}\right) a-\left(\mu-Q^{2}\right) \bar{a}$,

$N(a, \bar{a})=\left[a^{2}+2|a|^{2}+a|a|^{2}\right]$.

From Eqs. (9.46) and (9.70) we find that $a(x, \tau)$ also satisfies periodic boundary conditions on $[-\pi, \pi]$, and with (9.71) we can calculate the induced $\widetilde{O}(2)$ action on $a(x, \tau)$

$$
\begin{aligned}
& \left(\widetilde{T}_{d} \cdot A\right)(x, \tau)=A_{Q}(x ; \phi)\left(1+\left(T_{d} \cdot a\right)(x, \tau)\right) \\
& (\widetilde{\kappa}(\phi) \cdot A)(x, \tau)=A_{Q}(x ; \phi)(1+(C \kappa \cdot a)(x, \tau)) ;
\end{aligned}
$$

thus the $\widetilde{O}(2)$ action on $a(x, \tau)$ is generated by $T_{d}$ and $C \kappa$. Note that the $Z_{Q}$ action requires only symmetry with respect to $T_{2 \pi / Q}$, and this is contained in Eq. (9.72). The covariance of Eq. (9.71a) with respect to $T_{d}$ and $C \kappa$ is easily checked; this remaining $\widetilde{O}(2)$ symmetry will introduce nongeneric features into the secondary bifurcations. b. Linear stability for $a=0$

The linear operator $(9.71 b)$ is self-adjoint,

$$
\left\langle\mathcal{L} a_{1}, a_{2}\right\rangle=\left\langle a_{1}, \mathcal{L} a_{2}\right\rangle,
$$

with respect to the inner product

$\left\langle a_{1}, a_{2}\right\rangle=\frac{1}{2} \int_{-\pi}^{\pi} \frac{d x}{2 \pi}\left[\overline{a_{1}(x)} a_{2}(x)+a_{1}(x) \overline{a_{2}(x)}\right]$,

so we expect real eigenvalues $\lambda$ in the spectrum determined by

$$
\mathcal{L} v=\lambda v \text {. }
$$

By inspection the eigenfunctions $v$ are of the form

$v(x)=z_{1}(\lambda) e^{i k x}+z_{2}(\lambda) e^{-i k x}, \quad k=0,1,2,3, \ldots$,

with complex coefficients $\left(z_{1}, z_{2}\right)$ that satisfy

$$
\begin{aligned}
& {\left[\lambda+k^{2}+2 k Q+\left(\mu-Q^{2}\right)\right] z_{1}=-\left(\mu-Q^{2}\right) \bar{z}_{2} .} \\
& {\left[\lambda+k^{2}-2 k Q+\left(\mu-Q^{2}\right)\right] z_{2}=-\left(\mu-Q^{2}\right) \bar{z}_{1} .}
\end{aligned}
$$

The real and imaginary parts of $z_{i}, i=1,2$, satisfy Eq. (9.77) separately, since $\lambda$ is real; this decoupling is due to the symmetry and forces the eigenvalues to have double multiplicity when $k \neq 0$. More precisely, let $\left(r_{1}, r_{2}\right) \in \mathbb{R}^{2}$ be a real solution to Eq. (9.77) for eigenvector

$$
v(x)=r_{1}(\lambda) e^{i k x}+r_{2}(\lambda) e^{-i k x} .
$$

Then the translated eigenvector

$$
w(x)=\left(T_{\pi / 2 k} v\right)(x)=i r_{1}(\lambda) e^{i k x}-i r_{2}(\lambda) e^{-i k x}
$$

has imaginary coefficients and is linearly independent of $v(x)$; thus $\lambda$ has multiplicity two. [The other symmetry $C \kappa$ leaves $v(x)$ invariant.] When $k=0$ then Eqs. (9.76) and (9.77) yield only one (linearly independent) solution:

$$
v(x)=1, \lambda=-2\left(\mu-Q^{2}\right) .
$$

For a pure mode $A_{Q}$ to exist requires

$$
\left|A_{Q}\right|^{2}=\mu-Q^{2}>0 \text {, }
$$

so the $k=0$ mode $(9.79)$ is always stable. The possibilities for instability arise from Eq. (9.77) for $k \geq 1$. Without loss of generality let $\left(z_{1}, z_{2}\right)$ be real. Then a nontrivial solution $\left(r_{1} \neq 0, r_{2} \neq 0\right)$ requires $\lambda=\lambda_{+}$or $\lambda=\lambda_{-}$ where

$$
\lambda_{ \pm}=-\left(k^{2}+\mu-Q^{2}\right) \pm \sqrt{\left(\mu-Q^{2}\right)^{2}+(2 k Q)^{2}} .
$$

The $\lambda_{-}$eigenvalue is always negative [cf. Eq. (9.80)], but $\lambda_{+}$satisfies $\lambda_{+} \leq 0$ if and only if

$$
\mu-Q^{2} \geq 2 Q^{2}-\frac{1}{2} k^{2} \text {. }
$$

With periodic boundary conditions, $k_{\min }=1$ so the pure mode $A_{Q}$ will be linearly stable provided

$$
\left|A_{Q}\right|^{2}>2 Q^{2}-1 \text {. }
$$

Stated slightly differently, as $\mu$ increases above $\mu=0$, all 
pure modes with wave numbers in the band

$$
Q^{2}<\frac{1}{3}\left(\mu+\frac{1}{2}\right)
$$

are stable. Comparing Eqs. (9.67) and (9.84) we see that the finite-length periodic boundary condition is stabilizing in the sense that at fixed $\mu$ there is a wider band of allowed $Q$ values (Tuckerman and Barkley, 1990). With periodic boundary conditions, the Eckhaus instability corresponds to the $k=1$ instability that sets in when condition (9.83) fails. As $\mu$ decreases further below the Eckhaus boundary (9.84) there are additional instabilities of higher $k$ values, as shown by Eq. (9.82).

\section{c. Center-manifold reduction for $\lambda_{+} \approx 0$}

For fixed $k$ and $Q$, as $\mu$ decreases below $3 Q^{2}-\frac{1}{2} k^{2}$, the $\lambda_{+}$eigenvalue (9.81) crosses through zero moving from the left half-plane into the right half-plane. At $\lambda_{+}=0$ there are two critical modes corresponding to this eigenvalue,

$$
\begin{aligned}
& v_{+}(x)=r_{1}\left(\lambda_{+}\right) e^{i k x}+r_{2}\left(\lambda_{+}\right) e^{-i k x}, \\
& w_{+}(x)=i r_{1}\left(\lambda_{+}\right) e^{i k x}-i r_{2}\left(\lambda_{+}\right) e^{-i k x},
\end{aligned}
$$

so that the expansion of $a(x, t)$ [cf. Eq. (7.2)] requires two real amplitudes $(\alpha, \beta) \in E^{c}$,

$$
a(x, t)=\alpha(t) v_{+}(x)+\beta(t) \omega_{+}(x)+S(x, t),
$$

where

$$
\left\langle v_{+}, S\right\rangle=\left\langle w_{+}, S\right\rangle=0 .
$$

[Recall that $\mathcal{L}$ is self-adjoint; otherwise the projection in Eq. (9.86b) would require the appropriate adjoint eigenfunctions.] The center-manifold dynamics for $(\alpha, \beta)$ is more conveniently expressed by defining the complex amplitude

$$
z=\alpha+i \beta
$$

and rewriting Eq. (9.86)

$$
\begin{aligned}
& a(x, t)=z(t) v_{z}(x)+\bar{z}(t) v_{\bar{z}}(x)+S, \\
& v_{z}(x)=\frac{1}{2}\left[v_{+}(x)-i w_{+}(x)\right], \\
& v_{\bar{z}}(x)=\frac{1}{2}\left[v_{+}(x)+i w_{+}(x)\right] .
\end{aligned}
$$

With this notation $z(t)$ is analogous to $x_{1}$ in Eq. (7.2a) and $S(x, t)$ is analogous to $x_{2}$ in Eq. (7.2b); the equation for $\dot{z}$ follows from Eq. (9.87), which can be written as

$$
z=\frac{\left\langle v_{+}, a\right\rangle+i\left\langle w_{+}, a\right\rangle}{r_{1}\left(\lambda_{+}\right)^{2}+r_{2}\left(\lambda_{+}\right)^{2}}
$$

using $\left\langle v_{+}, v_{+}\right\rangle=\left\langle w_{+}, w_{+}\right\rangle=r_{1}^{2}+r_{2}^{2}$. Differentiating Eq. (9.89) and using Eq. (9.71) yields

$$
\begin{aligned}
\dot{z} & =\lambda_{+} z-\frac{\left(\mu-Q^{2}\right)}{\left(r_{1}^{2}+r_{2}^{2}\right)}\left[\left\langle v_{+}, N(a, \bar{a})\right\rangle+i\left\langle w_{+}, N(a, \bar{a})\right\rangle\right] \\
& =\lambda_{+} z-\frac{\left(\mu-Q^{2}\right)}{\left(r_{1}^{2}+r_{2}^{2}\right)} \int_{-\pi}^{\pi} \frac{d x}{2 \pi} e^{-i k x}\left[r_{1} N(a, \bar{a})+r_{2} \overline{N(a, \bar{a})}\right]
\end{aligned}
$$

Then from Eqs. (9.88) and (9.71) we obtain

$$
\begin{aligned}
\frac{\partial S}{\partial t}= & \mathcal{L} S-\left(\mu-Q^{2}\right) N(a, \bar{a})+\frac{\left(\mu-Q^{2}\right)}{r_{1}^{2}+r_{2}^{2}}\left\{v_{z}(x) \int_{-\pi}^{\pi} \frac{d x}{2 \pi} e^{-i k x}\left[r_{1} N(a, \bar{a})+r_{2} \overline{N(a, \bar{a})}\right]\right\} \\
& +v_{\bar{z}}(x) \int_{-\pi}^{\pi} \frac{d x}{2 \pi} e^{i k x}\left[r_{1} \overline{N(a, \bar{a})}+r_{2} N(a, \bar{a})\right] .
\end{aligned}
$$

The center manifold near $a=0$ is described by

$$
S(x, t)=h(x, z(t), \overline{z(t)}),
$$

where the function $h$ satisfies [cf. Eqs. (7.4) and (7.7)]

$$
\dot{z} \frac{\partial h}{\partial z}+\dot{\bar{z}} \frac{\partial h}{\partial \bar{z}}=\left.\frac{\partial S}{\partial t}\right|_{S=h}
$$

The Taylor expansion of $h$ beings at second order in $(z \bar{z})$,

$$
h(x, z, \bar{z})=h_{1}(x) z^{2}+h_{2}(x)|z|^{2}+h_{3}(x) \bar{z}^{2}+\mathcal{O}(3),
$$

with coefficients $h_{i}(x), i=1,2,3$, that are determined by applying Eq. (9.93) to (9.92) and requiring that the second-order terms balance. This procedure yields the following equations:

$$
\left[\frac{\partial^{2}}{\partial x^{2}}+2 i Q \frac{\partial}{\partial x}-\left(\mu-Q^{2}\right)-2 \lambda_{+}\right] h_{1}(x)-\left(\mu-Q^{2}\right) \overline{h_{3}(x)}=\left(\mu-Q^{2}\right)\left(r_{1}^{2}+2 r_{1} r_{2}\right) e^{i 2 k x},
$$




$$
\begin{aligned}
& {\left[\frac{\partial^{2}}{\partial x^{2}}+2 i Q \frac{\partial}{\partial x}-\left(\mu-Q^{2}\right)-2 \lambda_{+}\right] h_{2}(x)-\left(\mu-Q^{2}\right) \overline{h_{2}(x)}=2\left(\mu-Q^{2}\right)\left(r_{1} r_{2}+r_{1}^{2}+r_{2}^{2}\right),} \\
& {\left[\frac{\partial^{2}}{\partial x^{2}}+2 i Q \frac{\partial}{\partial x}-\left(\mu-Q^{2}\right)-2 \lambda_{+}\right] h_{3}(x)-\left(\mu-Q^{2}\right) \overline{h_{1}(x)}=\left(\mu-Q^{2}\right)\left(r_{2}^{2}+2 r_{1} r_{2}\right) e^{-i 2 k x},}
\end{aligned}
$$

which in turn yield solutions

$$
\begin{aligned}
& h_{1}(x)=\eta_{1} e^{i 2 k x}, \\
& h_{2}=-\frac{\left(\mu-Q^{2}\right)\left(r_{1} r_{2}+r_{1}^{2}+r_{2}^{2}\right)}{\mu-Q^{2}+\lambda_{+}}, \\
& h_{3}(x)=\eta_{3} e^{-i 2 k x},
\end{aligned}
$$

where $\left(\eta_{1}, \eta_{2}\right)$ satisfy

$$
\left[\begin{array}{cc}
(2 k)^{2}+4 Q k+\left(\mu-Q^{2}\right)+2 \lambda_{+} & \mu-Q^{2} \\
\mu-Q^{2} & (2 k)^{2}-4 Q k+\left(\mu-Q^{2}\right)+2 \lambda_{+}
\end{array}\right]\left[\begin{array}{l}
\eta_{1} \\
\eta_{3}
\end{array}\right]=-\left(\mu-Q^{2}\right)\left[\begin{array}{l}
r_{1}^{2}+2 r_{1} r_{2} \\
r_{2}^{2}+2 r_{1} r_{2}
\end{array}\right] .
$$

This approximation for $h(x, z, \bar{z})$ is adequate to determine the leading nonlinear terms in Eq. (9.90a). On the center manifold,

$$
a=z v_{z}+\bar{z} v_{\bar{z}}+\eta_{1} e^{i 2 k x} z^{2}+h_{2}|z|^{2}+\eta_{3} e^{-i 2 k x} \bar{z}^{2}+\cdots,
$$

so the nonlinearity in Eq. (9.90a) yields

$$
\int_{-\pi}^{\pi} \frac{d x}{2 \pi} e^{-i k x}\left[r_{1} N(a, \bar{a})+r_{2} \overline{N(a, \bar{a})}\right]=\sigma z|z|^{2}+\mathcal{O}\left(z|z|^{4}\right),
$$

where

$$
\sigma=4 h_{2}\left(r_{1}^{2}+r_{1} r_{2}+r_{2}^{2}\right)+4 r_{1} r_{2}\left(\eta_{1}+\eta_{2}\right)+2\left(\eta_{1} r_{1}^{2}+\eta_{3} r_{2}^{2}\right)+r_{1}^{2}\left(r_{1}^{2}+2 r_{2}^{2}\right)+r_{2}^{2}\left(r_{2}^{2}+2 r_{1}^{2}\right) .
$$

Thus the steady-state bifurcation from the pure-mode branch is described by

$$
\dot{z}=\lambda_{+} z-\frac{\left(\mu-Q^{2}\right) \sigma}{\left(r_{1}^{2}+r_{2}^{2}\right)} z|z|^{2}+\mathcal{O}\left(z|z|^{4}\right)
$$

and there are new branches of equilibria $(\dot{z}=0)$ satisfying

$$
|z|^{2} \approx \frac{\left(r_{1}^{2}+r_{2}^{2}\right) \lambda_{+}}{\left(\mu-Q^{2}\right) \sigma}
$$

with an arbitrary phase reflecting the translation symmetry $\widetilde{T}_{d}$ that has been broken.

The sign of $\sigma$ determines whether these new equilibria occur for $\lambda_{+}<0$ (subcritical) or $\lambda_{+}>0$ (supercritical). It is enough to determine this sign at $\lambda_{+}=0$, in which case the expressions for $r_{1}$ and $r_{2}$ from Eq. (9.77) simplify:

$$
r_{1}=-(2 Q-k), \quad r_{2}=2 Q+k
$$

(up to an overall normalization). In addition, at $\lambda_{+}=0$

$$
\mu-Q^{2}=-2 r_{1} r_{2}
$$

from Eq. (9.82) and the center-manifold coefficients (9.95) reduce to

$$
\eta_{1}=\frac{r_{1}^{2} r_{2}}{r_{1}+r_{2}}
$$

$$
\begin{aligned}
& h_{2}=-\left(r_{1}^{2}+r_{1} r_{2}+r_{2}^{2}\right), \\
& \eta_{3}=\frac{r_{1} r_{2}^{2}}{r_{1}+r_{2}} .
\end{aligned}
$$

With these formulas at criticality, the expression for $\sigma$ (9.97) simplifies considerably,

$$
\sigma=-3\left(r_{1}+r_{2}\right)^{2}\left(r_{1}^{2}+r_{2}^{2}\right), \quad\left(\lambda_{+}=0\right),
$$

so that Eq. (9.98) may be rewritten

$$
\begin{aligned}
\dot{z}= & \lambda_{+} z+3\left(\mu-Q^{2}\right)\left(r_{1}+r_{2}\right)^{2} z|z|^{2} \\
& +\mathcal{O}\left(\lambda_{+} z|z|^{2}, z|z|^{4}\right) .
\end{aligned}
$$

Since $\left(\mu-Q^{2}\right)\left(r_{1}+r_{2}\right)^{2}>0$, the new equilibria (9.99) are subcritical and unstable.

\section{OMITTED TOPICS}

The ideas of center-manifold theory and PoincaréBirkhoff normal forms are discussed by many authors. An introductory account is provided by Rasband (1990); for the reader seeking a more sophisticated treatment, both Guckenheimer and Holmes (1986), Chapter 3, and Arnold (1988a), Chapter 6, are suggested. The recent review by Vanderbauwhede (1989) provides very detailed proofs for the finite-dimensional theory, and a careful re- 
view of center manifolds in Banach spaces is provided by Vanderbauwhede and Iooss (1991). Additional material on the infinite-dimensional case in particular can be found in Marsden and McCracken (1976), Hassard et al. (1978), Ruelle (1989), and the encyclopedic volume by Chow and Hale (1982). Finally, there is the monograph by Iooss and Joseph (1989), which develops local bifurcation theory without using center manifolds.

In one-parameter systems, the Feigenbaum bifurcation and global bifurcations involving homoclinic and heteroclinic phenomena are important topics outside the scope of this review. References for the former topic include Cvitanovic (1984), Collett and Eckmann (1980), Lanford (1980), Vul et al. (1984), as well as the original papers by Feigenbaum (1978, 1979, 1980). Global bifurcations, especially Silnikov-type bifurcations and Melnikov theory, are discussed by Guckenheimer and Holmes (1986) and Wiggins $(1988,1990)$. In addition, the paper by Glendinning and Sparrow (1984) provides an accessible introduction to the Silnikov bifurcation.

The recent lecture by Arnold (1989) touches on many current research topics, in particular, multiparameter bifurcation problems and bifurcations in symmetric systems. The examples in Sec. IX were selected in part to illustrate the importance of these subjects. An introduction to codimension-two bifurcations (i.e., bifurcations typical for two-parameter systems) is provided by Guckenheimer and Holmes (1986), Chapter 7, and Arnold (1988a), Chapter 6, but much of the work in this area is scattered in the research literature; Golubitsky and Guckenheimer (1986) and Roberts and Stewart (1991) are two recent conference proceedings. Bifurcation theory for symmetric systems is likewise an actively developing subject. In addition to the recent reviews by Stewart (1988), Gaeta (1990), and Crawford and Knobloch (1991), there are the more extensive treatments in Vanderbauwhede (1982), Sattinger (1983), and Golubitsky, Stewart, and Schaeffer (1988).

Hamiltonian bifurcation theory is an important subject that is neglected here altogether. Unfortunately, there does not appear to be a systematic discussion of this theory for nonmathematicians at a level comparable to this review, and the literature is extensive. For bifurcation from equilibria of flows, Chapter 8 in Abraham and Marsden (1978) is a possible starting point, in addition to the brief overviews by Meyer (1975, 1986). Up-to-date discussions of Hamiltonian normal-form theory can be found in Bryuno (1988) and van der Meer (1985). This latter monograph treats the so-called Hamiltonian Hopf bifurcation in detail. Howard and MacKay (1987) give a nice discussion of the linear instabilities encountered in symplectic maps; Golubitsky and Stewart (1987) describe a generic setting for bifurcation in symmetric Hamiltonian systems. The closely related subject of bifurcation theory for reversible systems is showing a rapid development. Recent reviews have been given by Arnold and Sevryuh (1986) and Roberts and Quispel (1991).

Finally, we mention the authoritative volumes emerg- ing from the "Kolmogorov school:" Anosov and Arnold (1988), Sinai (1989), Arnold (1988b), and Arnold and Novikov (1990), which provide many references to the Soviet literature. In particular, Anosov and Arnold (1988) treat normal forms and invariant manifold theory, and Arnold (1988b) discusses Hamiltonian normal forms and bifurcation.

\section{ACKNOWLEDGMENTS}

I am grateful to P. Morrison for arranging my visit to the Institute for Fusion Studies and to R. Hazeltine for suggesting this review. C. Kueny, W. Saphir, and B. Shadwick assisted in writing notes for the original lectures. I would like to thank M. Silber and especially A. Kaufman for suggesting improvements in the final manuscript. This work was supported in part by the U.S. Department of Energy Grant No. DE-FG05-80ET-53088 at the IFS and in part at the Institute for Nonlinear Science at The University of California, San Diego by DARPA Applied and Computational Mathematics Program Contract No. F49620-87-C-0117 and by the DARPA University Research Initiative Contract No. N001486-K-0758.

\section{INDEX}

$\begin{array}{ll}\text { asymptotic stability } & 995,997 \\ \text { bifurcation } & 992 \\ \text { degenerate } & 1021,1025 \\ \text { Hopf } & 997,998,1003,1006,1022 \\ \text { imperfect } & 1003 \\ \text { period-doubling } & 998,1005 \\ \text { pitchfork } & 997,998,1002,1005 \\ \text { saddle-node } & 997,998,1000,1005 \\ \text { steady-state } & 997,998,1000,1004,1026 \\ \text { subcritical } & 1004 \\ \text { supercritical } & 1004 \\ \text { transcritical } & 997,998,1001,1005 \\ \text { bifurcation diagram } & 1001 \\ \text { bifurcation set } & 996 \\ \text { bifurcation surface } & 1025 \\ \text { bifurcation theory } & 992 \\ \text { branch of solutions } & 999 \\ \text { center manifold } & 1008,1009 \\ \text { local attractivity } & 1008 \\ \text { nonuniqueness } & 1008,1012 \\ \text { reduction to } & 1009 \\ \text { center subspace } & 995,997 \\ \text { codimension } & 992,1025 \\ \text { critical system } & 996 \\ \text { dynamical system } & 992 \\ \text { Eckhaus instability } & 1021,1031,1032 \\ \text { eigenspace } & 995 \\ \text { eigenvalue } & 994 \\ \text { degenerate } & 1000 \\ \text { simple } & \end{array}$




\begin{tabular}{|c|c|}
\hline fixed point & 993 \\
\hline hyperbolic & 996,997 \\
\hline Floquet theory & 997 \\
\hline flow & 993 \\
\hline generic & 992 \\
\hline graph representation & 1010,1012 \\
\hline Ginzburg-Landau equation & 1027 \\
\hline Hartman-Grobman theorem & 996,997 \\
\hline homeomorphism & 996 \\
\hline homological equation & 1014 \\
\hline \multicolumn{2}{|l|}{ Hopf bifurcation } \\
\hline flows & 1003 \\
\hline maps & 1006 \\
\hline implicit function theorem & 999 \\
\hline invariance equation & 1010,1012 \\
\hline invariant circle & 1007 \\
\hline invariant manifold & 1007 \\
\hline invariant subspace & 994, 997 \\
\hline invariant torus & 1007 \\
\hline Liapunov-Schmidt reduction & 1007 \\
\hline Lie bracket & 1014 \\
\hline map & 993 \\
\hline mode-locking & 1007 \\
\hline node & 1001 \\
\hline nonwandering point & 1009 \\
\hline normal form & 998 \\
\hline dynamics & 1000 \\
\hline symmetry & 1017,1019 \\
\hline unfolding & 998 \\
\hline period-doubling bifurcation & 898,1005 \\
\hline phase portrait & 992 \\
\hline phase-shift symmetry & 1003,1021 \\
\hline phase space & 992 \\
\hline \multicolumn{2}{|l|}{ pitchfork bifurcation } \\
\hline flows & 997,1002 \\
\hline maps & 998,1005 \\
\hline recurrent point & 1009 \\
\hline reflection symmetry & 1002 \\
\hline return map & 993 \\
\hline saddle & 1001 \\
\hline \multicolumn{2}{|l|}{ saddle-node bifurcation } \\
\hline flows & 997,1000 \\
\hline maps & 998,1005 \\
\hline Shoshitaishvili theorem & 1011 \\
\hline stable manifold & 1008,1009 \\
\hline stable subspace & 995,997 \\
\hline \multicolumn{2}{|l|}{ steady-state bifurcation } \\
\hline flows & 997,1000 \\
\hline maps & 998,1004 \\
\hline stroboscopic map & 993 \\
\hline subcritical & 1004 \\
\hline supercritical & 1004 \\
\hline suspended system & 1012 \\
\hline topological conjugacy & 996 \\
\hline trajectory & 993 \\
\hline unstable manifold & 1008,1009 \\
\hline unstable subspace & 995,997 \\
\hline vector field & 993 \\
\hline
\end{tabular}

\section{REFERENCES}

Abraham, R., and J. Marsden, 1978, Foundations of Mechanics (Benjamin-Cummings, Reading, MA).

Ahlers, G., 1989, "Experiments in bifurcations and onedimensional patterns in nonlinear systems far from equilibrium," in Lectures in the Sciences of Complexity 1, edited by D. L. Stein (Addison-Wesley, Redwood City, CA), p. 175.

Ahlers, G., D. Cannell, M. Dominguez-Lerma, and R. Heinrichs, 1986, "Wavenumber selection and Eckhaus instability in Couette-Taylor flow," Physica D 23, 202.

Anosov, D. V., and V. I. Arnold, 1988, Eds., Dynamical Systems I: Ordinary Differential Equations and Smooth Dynamical Systems, Encyclopedia of Mathematical Sciences 1 (Springer, New York).

Arnold, V. I., 1972, "Lectures on bifurcation in versal families," Russ. Math. Surveys 27, 54.

Arnold, V. I., 1973, Ordinary Differential Equations (MIT, Cambridge, MA).

Arnold, V. I., 1988a, Geometrical Methods in the Theory of Ordinary Differential Equations, second edition (Springer, New York.

Arnold, V. I., 1988b, Ed., Dynamical Systems III: Mathematical Aspects of Classical and Celestial Mechanics, Encyclopedia of Mathematical Sciences 3 (Springer, New York).

Arnold, V. I., 1989, "Bifurcation and singularities in mathematics and mechanics," in Theoretical and Applied Mechanics, Proceedings of the 17th International Congress of Theoretical and Applied Mechanics, Grenoble, 1988, edited by P. Germain, M. Piau, and D. Caillerie (North-Holland, Elsevier, Amsterdam/New York), p. 1.

Arnold, V. I., and S. P. Novikov, 1990, Eds., Dynamical Systems IV: Symplectic Geometry and its Applications, Encyclopedia of Mathematical Sciences 4 (Springer, New York).

Arnold, V. I., and M. B. Sevryuk, 1986, "Oscillations and bifurcations in reversible systems," in Nonlinear Phenomena in Plasma Physics and Hydrodynamics, edited by R. Z. Sagdeev (Mir, Moscow), p. 31.

Belitskii, G. R., 1978, "Equivalence and normal forms of germs of smooth mappings," Russ. Math. Surveys 33, 107.

Belitskii, G. R., 1981, "Normal forms relative to the filtering action of a group," Trans. Mosc. Math. Soc. 40, 1.

Brand, H. R., 1988, "Phase dynamics - a review and a perspective," in Propagation in Systems Far from Equilibrium, edited by J. E. Wesfreid, H. R. Brand, P. Manneville, G. Albinet, and N. Boccara (Springer, New York), p. 206.

Bryuno, A. D., 1988, "The normal form of a Hamiltonian system," Russ. Math. Surveys 43, 25.

Carr, J., 1981, Applications of Center Manifold Theory (Springer, New York).

Chillingworth, D., 1976, Differentiable Topology with a View to Applications (Pitman, London).

Chossat, P., and M. Golubitsky, 1987, "Hopf bifurcation in the presence of symmetry, center manifold and Liapunov-Schmidt reduction," in Oscillation, Bifurcation and Chaos, CMS Conf. Proc. 8, edited by F. Atkinson, W. Langford, and A. Mingarelli (American Mathematical Society, Providence), p. 343.

Chow, S. N., and J. K. Hale, 1982, Methods of Bifurcation Theory (Springer, New York).

Collett, P., and J-P. Eckmann, 1980, Iterated Maps on the Interval as Dynamical Systems (Birkhäuser, Basel).

Collett, P., and J-P. Eckmann, 1990, Instabilities and Fronts in Extended Systems (Princeton University, Princeton, NJ). 
Coullet, P., and E. Spiegel, 1987, "Evolution equations for extended systems," in Energy Stability and Convection, Pitman Research Notes 168, edited by G. P. Galdi and B. Straughan (Wiley, New York), p. 22.

Crawford, J. D., 1983, "Hopf bifurcation and plasma instabilities," Ph.D. thesis (University of California, Berkeley).

Crawford, J. D., and E. Knobloch, 1991, "Symmetry and symmetry breaking bifurcations in fluid dynamics," Annu. Rev. Fluid. Mech. 23, 341.

Crawford, J. D., E. Knobloch, and H. Riecke, 1990, "Perioddoubling mode interactions with circular symmetry," Physica D 44, 340.

Cross, M. C., and A. C. Newell, 1984, "Convection patterns in large aspect ratio systems," Physica D 10, 299.

Cushman, R., and J. A. Sanders, 1986, "Nilpotent normal forms and representation theory of $s l(2, R)$," in Multiparameter Bifurcation Theory, Contemporary Mathematics 56, edited by $\mathbf{M}$. Golubitsky and J. Guckenheimer (American Mathematical Society, Providence), p. 31.

Cvitanovic, P., 1984, Ed., Universality in Chaos (Hilger, Bristol). Eckhaus, W., 1965, Studies in Nonlinear Stability Theory (Springer, New York,).

Edwards, W. Stuart, 1990, "Linear spirals in the finite CouetteTaylor problem," in Instability and Transition Vol. 2, edited by M. Y. Hussaini and R. G. Voigt (Springer, New York), p. 408.

Elphick, C., E. Tirapegui, M. E. Brachet, P. Coullet, and G. Iooss, 1987 "A simple global characterization for normal forms of singular vector fields," Physica D 29, 95; 32, 488 (Addendum).

Euler, L., 1744, Additamentum I de Curvis Elasticis, Methodus Inveniendi Lineas Curvas Maximi Minimivi Proprietate Gandentes. In Opera Ommia I (1960), Vol. 24 (Füssli, Zurich), p. 231.

Feigenbaum, M., 1978 "Quantitative universality for a class of nonlinear transformations," J. Stat. Phys. 19, 25.

Feigenbaum, M., 1979, "The universal metric properties of nonlinear transformations," J. Stat. Phys. 21, 669.

Feigenbaum, M., 1980, "Universal behavior in nonlinear systems," Los Alamos Sci. 1, 4.

Gaeta, G., 1990, "Bifurcation and symmetry breaking," Phys. Rep. 189, 1.

Glendinning, P., and C. Sparrow, 1984, "Local and global behavior near homoclinic orbits," J. Stat. Phys. 35, 645.

Golubitsky, M., and J. Guckenheimer, 1986, Eds., Multiparameter Bifurcation Theory, Contemp. Math. 56 (American Mathematical Society, Providence).

Golubitsky, M., and W. Langford, 1981, "Classification and unfoldings of degenerate Hopf bifurcations," J. Diff. Eqns. 41, 375.

Golubitsky, M., and D. Schaeffer, 1985, Singularities and Groups in Bifurcation Theory, Vol. I (Springer, New York).

Golubitsky, M., and I. Stewart, 1987, "Generic bifurcation of Hamiltonian systems with symmetry," Physica D 24, 391.

Golubitsky, M., I. Stewart, and D. Schaeffer, 1988, Singularities and Groups in Bifurcation Theory, Vol. II (Springer, New York).

Graham, R., and J. A. Domaradzki, 1982, "Local amplitude equation of Taylor vortices and its boundary condition," Phys. Rev. A 26, 1572.

Guckenheimer, J., and P. Holmes, 1986, Nonlinear Oscillations, Dynamical Systems, and Bifurcations of Vector Fields (Springer, New York).

Guillemin, V., and A. Pollack, 1974, Differential Topology (Prentice-Hall, Englewood Cliffs, NJ).
Hall, P., 1980, "Centrifugal instabilities of circumferential flows in finite cylinders: nonlinear theory," Proc. R. Soc. London, Ser. A 372, 317.

Hartman, P., 1982, Ordinary Differential Equations (Birkhäuser, Boston).

Hassard, B., N. Kazarinoff, and Y.-H. Wan, 1978, Theory and Applications of Hopf Bifurcation, London Mathematical Society Lecture Notes 41 (Cambridge University, Cambridge, England).

Hirsch, M., C. Pugh, and M. Shub, 1977, Invariant Manifolds, Springer Lecture Notes in Mathematics No. 583 (Springer, New York).

Hirsch, M., and S. Smale, 1974, Differential Equations, Dynamical Systems, and Linear Algebra (Academic, New York).

Howard, J. E., and R. S. MacKay, 1987, "Linear stability of symplectic maps," J. Math. Phys. 28, 1036.

Hughes, D., and M. Proctor, 1990, "Chaos and the effect of noise in a model of three-wave mode coupling," Physica D 46, 163.

Iooss, G., and D. Joseph, 1989, Elementary Stability and Bifurcation Theory (Springer, New York).

Irwin, M. C., 1980, Smooth Dynamical Systems (Academic, New York).

Jordan, D. W., and P. Smith, 1987, Nonlinear Ordinary Differential Equations (Oxford University, New York/London).

Kramer, L., and W. Zimmerman, 1985, "On the Eckhaus instability for spatially periodic patterns," Physica D 16, 221.

Lanford, O., 1973, "Bifurcation of periodic solutions into invariant tori: the work of Ruelle and Takens," in Nonlinear Problems in the Physical Sciences and Biology, edited by I. Stakgold, D. D. Joseph, and D. H. Sattinger, Springer Lecture Notes in Mathematics No. 322 (Springer, Berlin/ Heidelberg/New York), p. 159.

Lanford, O., 1980, "Smooth transformations of intervals," in Séminaire Bourbaki 1980/81, Springer Lecture Notes in Mathematics No. 901 (Springer, Berlin/Heidelberg/New York), p. 36.

Lanford, O., 1983, "Introduction to the mathematical theory of dynamical systems," in Chaotic Behavior of Deterministic Systems, edited by G. Iooss, R. Helleman, and R. Stora (NorthHolland, Amsterdam), p. 3.

Langer, J. S., 1986, "Lectures in the Theory of Pattern Formation," in Chance and Matter, edited by J. Souletie, J. Vannimenus, and R. Stora (North-Holland, Amsterdam), p. 629.

Mallet-Paret, J., and J. Yorke, 1982, "Snakes: oriented families of periodic orbits, their sources, sinks, and continuation," J. Diff. Eqns. 43, 419.

Manneville, P., 1990, Dissipative Structures and Weak Turbulence (Academic, Boston).

Marsden, J., 1979, "On the geometry of the Liapunov-Schmidt procedure," in Global Analysis, edited by M. Grmela and J. E. Marsden, Springer Lecture Notes in Mathematics No. 755 (Springer, New York), p. 77.

Marsden, J., and M. McCracken, 1976, The Hopf Bifurcation and its Applications (Springer, New York).

Meunier, C., M. N. Bussac, and G. Laval, 1982, "Intermittency at the onset of stochasticity in nonlinear resonant coupling process," Physica D 4, 236.

Meyer, K., 1975, "Generic bifurcations in Hamiltonian systems," in Dynamical Systems - Warwick, 1974, edited by A. Manning, Springer Lecture Notes in Mathematics No. 468 (Springer, New York), p. 36.

Meyer, K., 1986, "Bibliographic notes on generic bifurcations 
in Hamiltonian systems," in Multiparameter Bifurcation Theory, edited by M. Golubitsky and J. Guckenheimer (American Mathematical Society, Providence), p. 373.

Newell, A., 1989, "The dynamics and analysis of patterns," in Lectures in the Sciences of Complexity 1, edited by D. L. Stein (Addison-Wesley, Redwood City, CA), p. 107.

Newell, A., T. Passot, and M. Souli, 1990, "The phase diffusion and mean drift equations for convection at finite Rayleigh numbers in large containers," J. Fluid Mech. 220, 187.

Olver, P., 1986, Applications of Lie Groups to Differential Equations (Springer, New York).

Pliss, V. A., 1964, "A reduction principle in stability theory of motions," Izv. Akad. Nauk SSSR, Ser. Matem. 28, 1297.

Pomeau, Y., and P. Manneville, 1979, "Stability and fluctuations of a spatially periodic convective flow," J. Phys. (Paris) Lett 40, 609.

Rasband, S. Neil, 1990, Chaotic Dynamics of Nonlinear Systems (Wiley, New York).

Riecke, H., and H.-G. Paap, 1986, "Stability and wave-vector restriction of axisymmetric Taylor vortex flow," Phys. Rev. A 33, 547.

Roberts, J. A. G., and G. R. W. Quispel, 1991, "Chaos and time-reversal symmetry," Phys. Rep., in press.

Roberts, M., and I. Stewart, 1991, Eds., Singularity Theory and Its Applications: Warwick 1989, Vol. 2: Singularities, Bifurcations and Dynamics, Springer Lecture Notes in Mathematics No. 1463 (Springer, Berlin/New York).

Ruelle, D., 1989, Elements of Differentiable Dyanmics of Bifurcation Theory (Academic, New York).

Ruelle, D., and F. Takens, 1971, "On the nature of turbulence," Commun. Math. Phys. 20, 167; Note concerning our paper "On the nature of turbulence," Commun. Math. Phys. 23, 343.

Sattinger, D., 1983, "Branching in the presence of symmetry," CBMS-NSF Conference Notes 40 (SIAM, Philadelphia).

Shoshitaishvili, A. N., 1972, "Bifurcations of topological type at singular points of parametrized vector fields," Funct. Anal. Appl. 6, 169.

Shoshitaishvili, A. N., 1975, "The bifurcation of the topological type of the singular points of vector fields that depend on parameters," Trudy Seminara I. G. Petrovskogo 1, 279.

Shub, M., 1987, Global Stability of Dynamical Systems (Springer, New York).

Sijbrand, J., 1985, "Properties of center manifolds," Trans. Am. Math. Soc. 289, 431.

Sinai, Ya. G., 1989, Ed., Dynamical Systems II: Ergodic Theory with Applications to Dynamical Systems and Statistical Mechanics, Encyclopedia of Mathematical Sciences 2, (Springer, New York).

Sparrow, C., 1982, The Lorenz Equations (Springer, New York). Spivak, M., 1965, Calculus on Manifolds (Benjamin, Menlo Park, CA).

Stakgold, I., 1979, Green's Functions and Boundary Value Problems (Wiley, New York).

Stewart, I., 1988, "Bifurcations with Symmetry," in New Directions in Dynamical Systems, edited by T. Bedford and J. Swift (Cambridge University, Cambridge, England), p. 235.

Taylor, G. I., 1923, "Stability of a viscous liquid contained between two rotating cyclinders," Philos. Trans. R. Soc. London, Ser. A 233, 289.

Tsiveriotis, K., and R. A. Brown, 1989, "Bifurcation structure and the Eckhaus instability," Phys. Rev. Lett. 63, 2048.

Tuckerman, L., and D. Barkley, 1990, "Bifurcation analysis of the Eckhaus instability," Physica D 46, 57.

Vanderbauwhede, A., 1982, Local Bifurcation and Symmetry, Pitman Research Notes in Mathematics 75 (Pitman, Boston).

Vanderbauwhede, A., 1989, "Centre manifolds, Normal forms, and Elementary bifurcations," Dynamics Reported 2, 89.

Vanderbauwhede, A., and G. Iooss, 1991, "Centre manifold theory in infinite dimensions," Dynamics Reported, in press.

van der Meer, J. C., 1985, The Hamiltonian Hopf Bifurcation, Springer Lecture Notees in Mathematics No. 1160 (Springer, New York).

Verhulst, F., 1990, Nonlinear Differential Equations and Dynamical Systems (Springer, New York).

Vul, E., Ya. Sinai, and K. Khanin, 1984, "Feigenbaum universality and the thermodynamic formalism," Russ. Math. Surveys 39, 41.

Vyshkind, S., and M. Rabinovich, 1976, "The phase stochastization mechanism and the structure of wave turbulence in dissipative media," Sov. Phys. JETP 44, 292.

Wersinger, J.-M., J. Finn, and E. Ott, 1980, "Bifurcation and "strange" behavior in instability saturation by nonlinear three-wave mode coupling," Phys. Fluids 23, 1142.

Wiggins, S., 1988, Global Bifurcations and Chaos: Analytical Methods (Springer, New York).

Wiggins, S., 1990, Introduction to Applied Dynamical Systems and Chaos (Springer, New York).

Yorke, J., and K. Alligood, 1983, "Cascades of period-doubling bifurcations: a prerequisite for horseshoes," Bull. Am. Math. Soc. 9, 319. 